\title{
Hunting for Undetectable Metamorphic Viruses
}

\author{
A Project Report \\ Presented to \\ The Faculty of the Department of Computer Science \\ San Jose State University
}

\author{
In Partial Fulfillment \\ of the Requirements for the Degree \\ Master of Computer Science
}

by
Da Lin
December 2009 


\title{
Abstract \\ Hunting for Undetectable Metamorphic Viruses
}

\author{
by Da Lin
}

Commercial anti-virus scanners are generally signature based, that is, they scan for known patterns to determine whether a file is infected by a virus or not. To evade signature-based detection, virus writers have adopted code obfuscation techniques to create highly metamorphic computer viruses. Since metamorphic viruses change their appearance from generation to generation, signature-based scanners cannot detect all instances of such viruses.

To combat metamorphic viruses, detection tools based on statistical analysis have been studied. A tool based on hidden Markov models (HMMs) was previously developed and the results are encouraging - it has been shown that metamorphic viruses created by a well-designed metamorphic engine can be detected using an HMM.

In this project, we explore whether there are any exploitable weaknesses in this HMM-based detection approach. We create a highly metamorphic virus generating tool designed specifically to evade HMM-based detection. We then test our engine, showing that we can generate viral copies that cannot be detected using previously-developed HMM-based detection techniques. Finally, we consider possible defenses against our approach. 


\section{ACKNOWLEDGEMENTS}

I would like to thank Dr. Mark Stamp for trusting me with his idea. A special thanks is also owed to Dr. Stamp for his guidance, encouragement, and support throughout the duration of the project. 


\section{TABLE OF CONTENTS}

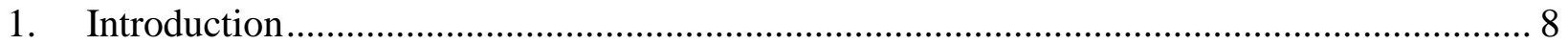

2. Computer Virus Evolution and Detection.................................................................. 9

2.1 Antivirus Defense Techniques ............................................................................. 10

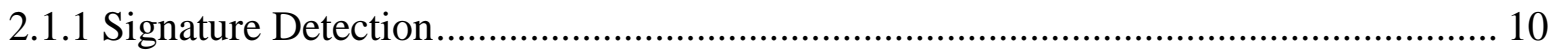

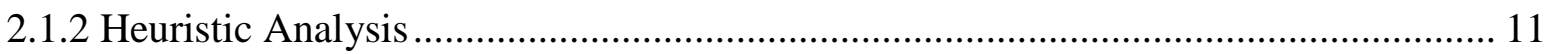

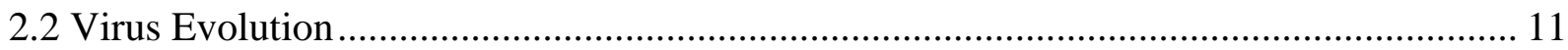

2.2.1 Virus Obfuscation Techniques................................................................... 11

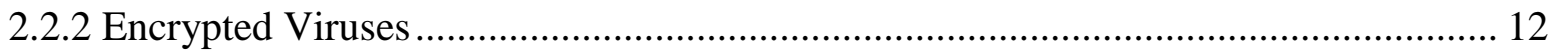

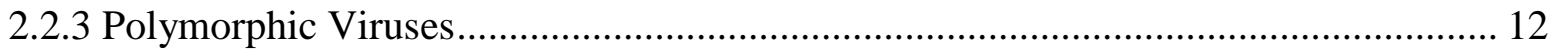

2.2.4 Metamorphic Viruses.................................................................................. 12

2.2.5 Formal Grammar Mutation ............................................................................... 17

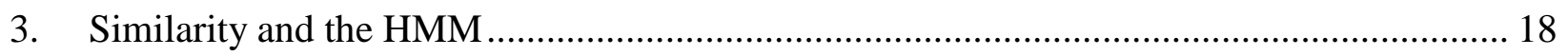

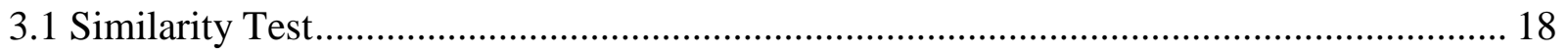

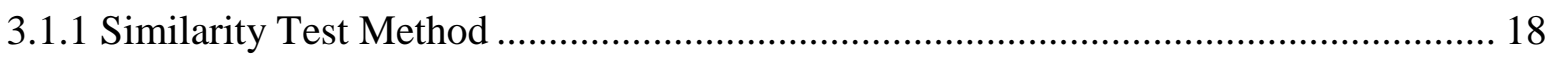

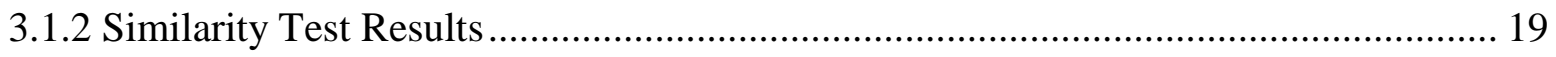

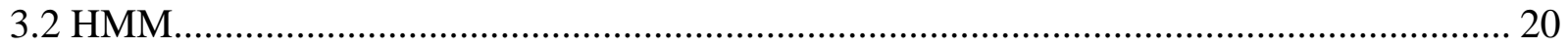

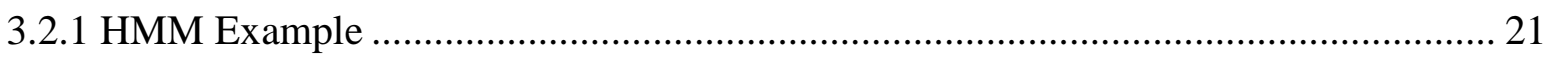

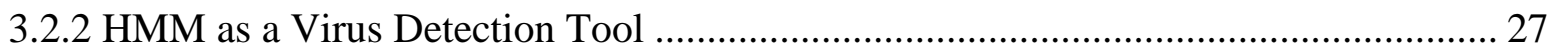

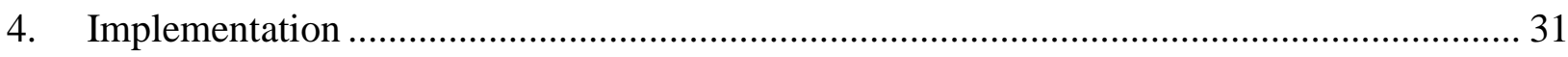

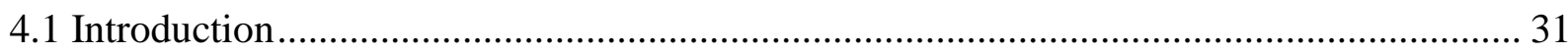

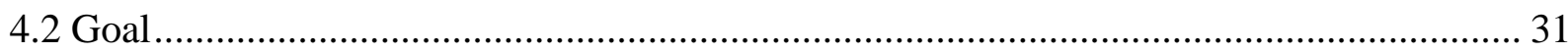

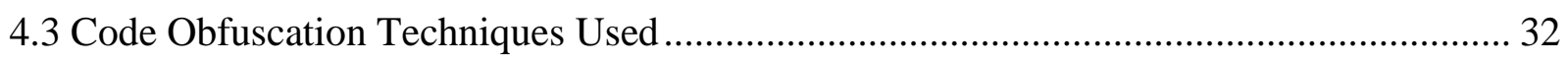

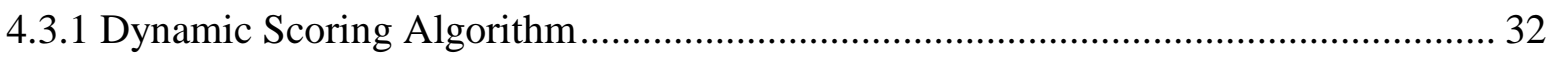

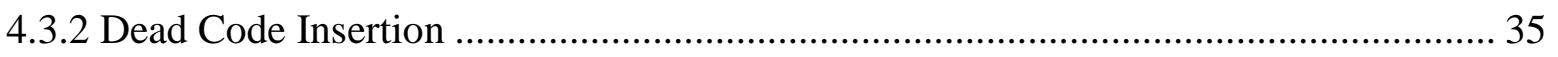

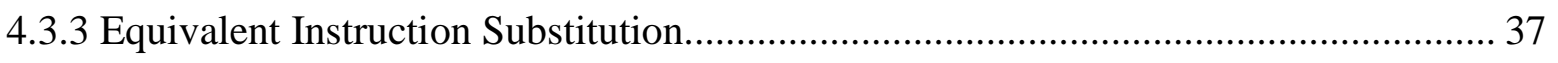

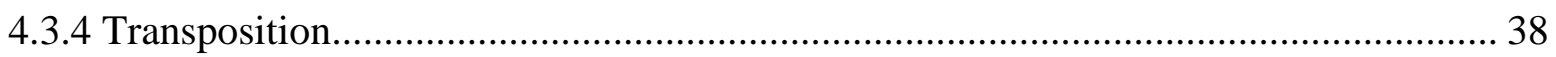

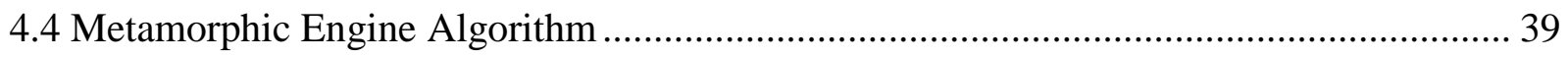

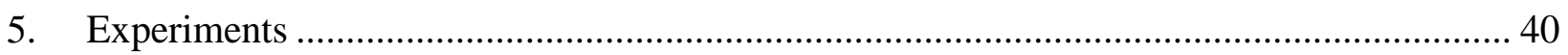

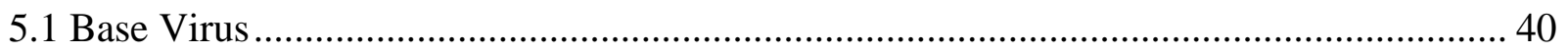


5.2 Similarity Test

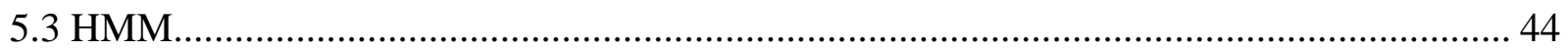

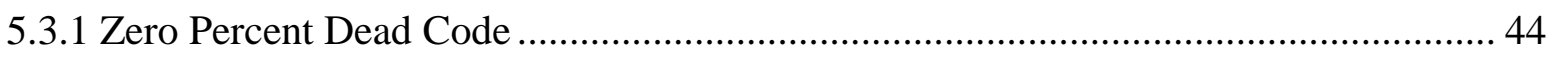

5.3.2 Copying Blocks of Dead Code from Normal File ........................................................ 45

5.3.3 Copying Subroutines and Blocks of Dead Code from Normal File ............................ 47

5.3.4 Copying Subroutines Only from Normal File ............................................................... 49

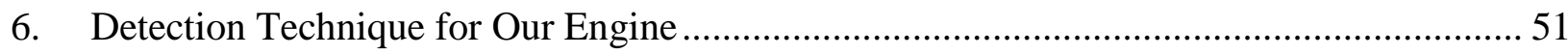

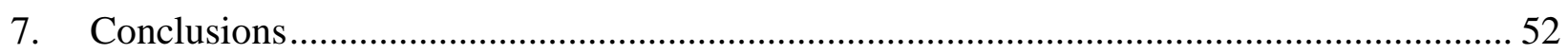

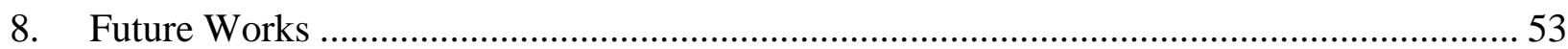

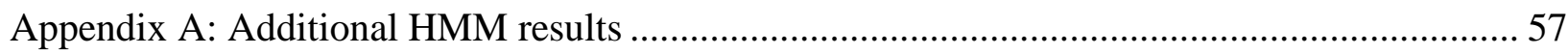

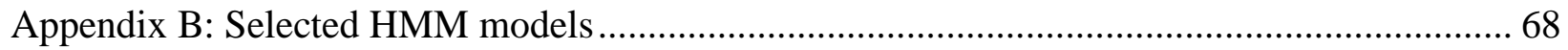

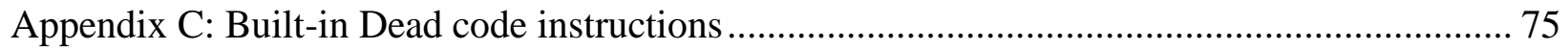

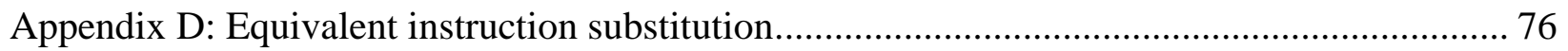




\section{LIST OF FIGURES}

Figure 1. A virus can spell doom for your computer [24] ..................................................... 10

Figure 2. Multiple shapes of a metamorphic virus body [20] ................................................. 13

Figure 3. Two different generations of RegSwap [9] ................................................................. 14

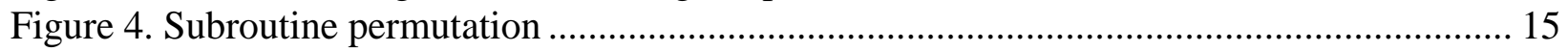

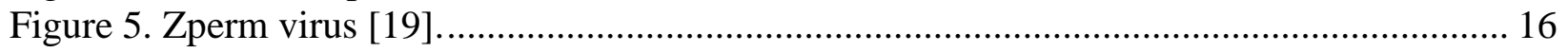

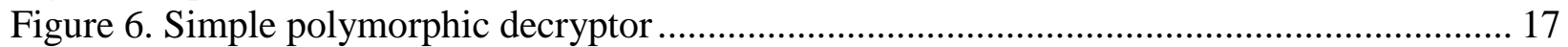

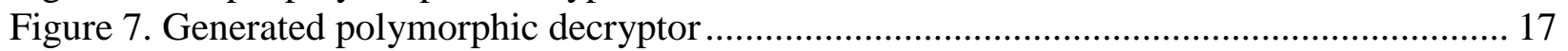

Figure 8. Process of finding the similarity between two assembly programs [2] ........................ 19

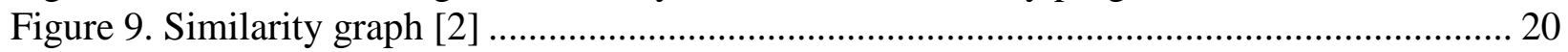

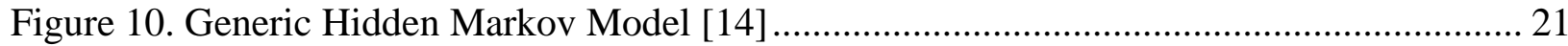

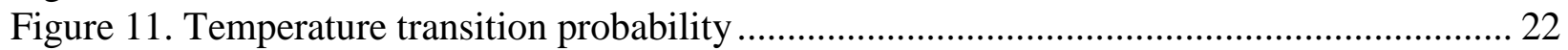

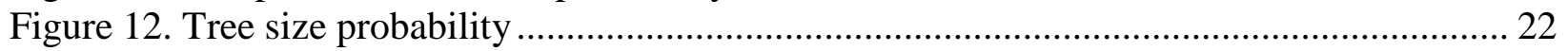

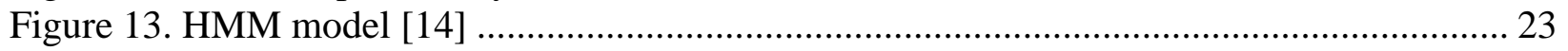

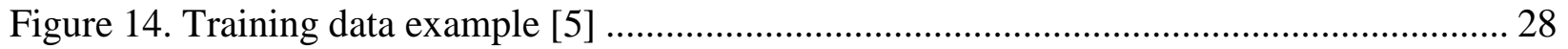

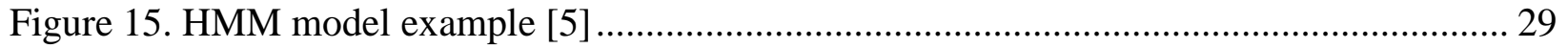

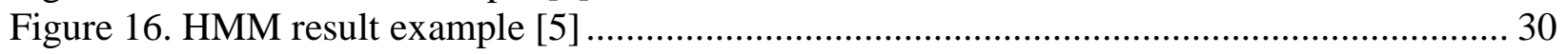

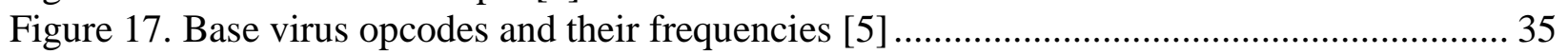

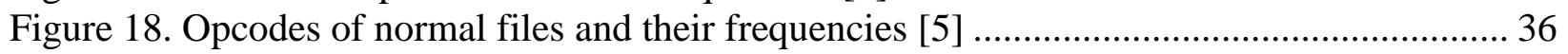

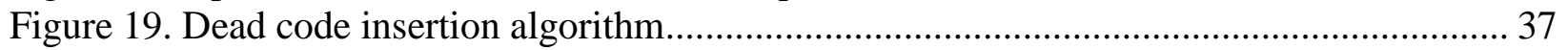

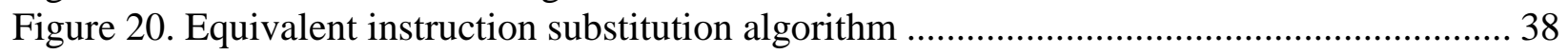

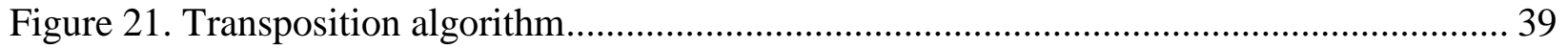

Figure 22. Metamorphic engine algorithm ............................................................................ 40

Figure 23. HMM results for base viruses generated by NGVCK ......................................... 41

Figure 24. Similarity score for morphed virus against normal files ............................................ 42

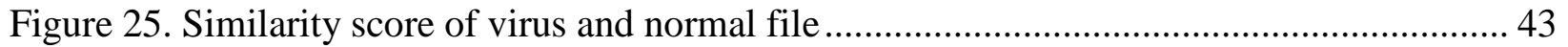

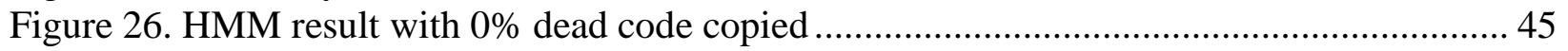

Figure 27. Maximun normal file scores vs. percentage increase ................................................... 46

Figure 28. HMM results with 35\% dead code copied .............................................................. 47

Figure 29. HMM result with 35\% dead code blocks and 5\% subroutine copied........................... 49

Figure 30. HMM results with 5\% subroutine copied................................................................ 50

Figure 31. Ave. scores vs. subroutines copied....................................................................... 52

Figure 32. Average scores vs. percent of dead code copied ....................................................... 53

Figure 33. HMM results with 10\% dead code copied ................................................................ 58

Figure 34. HMM results with 25\% dead code copied ................................................................ 59

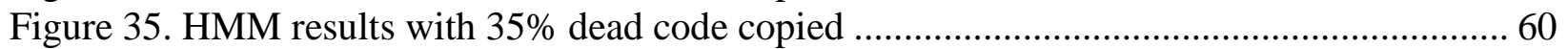

Figure 36. HMM results with 35\% dead code blocks and 15\% subroutines copied ...................... 62

Figure 37. HMM results with 35\% dead code blocks and 20\% subroutines copied ..................... 63

Figure 38. HMM results with 35\% dead code blocks and 30\% subroutines copied ...................... 65

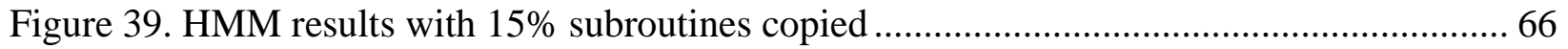

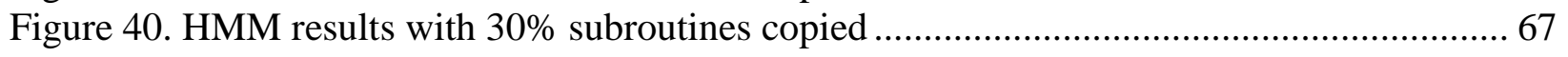




\section{LIST OF TABLES}

Table 1. Examples of instruction substitution used by W32/MetaPhor virus [19] ..................... 16

Table 2. Probabilities of observing (S, M, S, L) for all possible state sequences [14] ................ 24

Table 3. Opcode sequences of virus file and normal files ................................................... 33

Table 4. Opcode and opcode-pair counts lists ..................................................................... 33

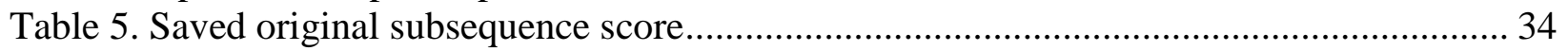

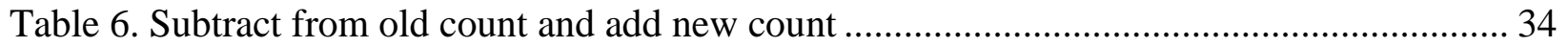

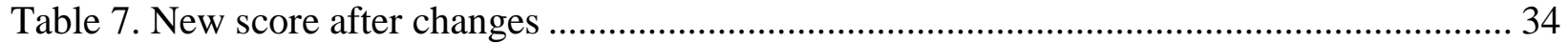

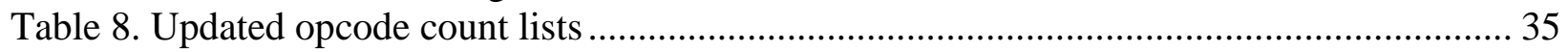

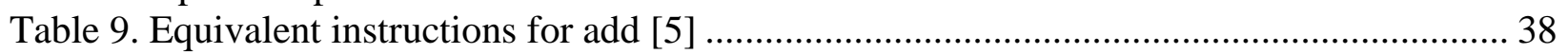

Table 10. Similarity score of virus and its peer normal file................................................. 43

Table 11. HMM Results with $0 \%$ dead code copied ........................................................ 45

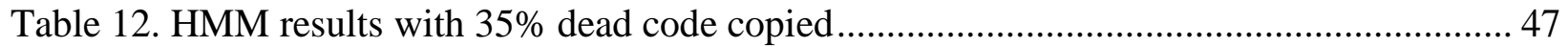

Table 13. HMM results with 35\% dead code blocks and 5\% subroutine copied ...................... 48

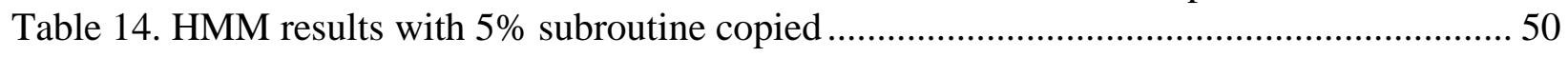

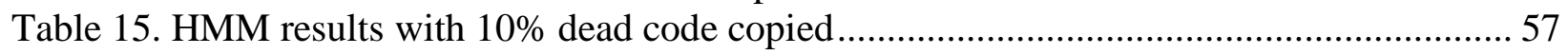

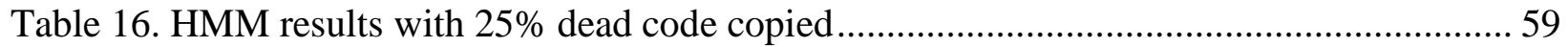

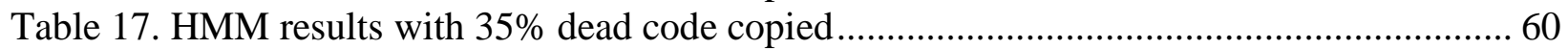

Table 18. HMM results with 35\% dead code blocks and $15 \%$ subroutine copied ..................... 61

Table 19. HMM results with 35\% dead code blocks and 20\% subroutines copied .................... 63

Table 20. HMM results with 35\% dead code blocks and 30\% subroutines copied.................... 64

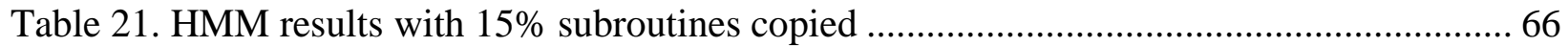

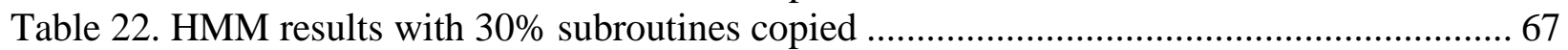

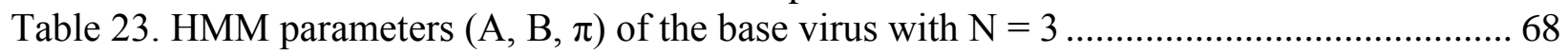

Table 24. HMM parameters $(A, B, \pi)$ of the virus without dead code copying with $\mathrm{N}=3 \ldots \ldots . .70$

Table 25. HMM parameters $(\mathrm{A}, \mathrm{B}, \pi)$ of the virus with most dead code copied with $\mathrm{N}=3 \ldots \ldots .72$ 


\section{Introduction}

A virus is a program designed to infect and potentially damage files on a computer [8]. To replicate itself, a virus must be permitted to execute code and write to memory. For this reason, many viruses attach themselves to executable files that are part of legitimate programs [16]. When an infected program is launched, the embedded virus is also executed and may replicate itself to infect other files and programs.

In general, a virus performs activities without permission of users. Some viruses may perform damaging activities on the host machine, such as corrupting hard disk data or crashing the computer. Other viruses are harmless and might, for example, print annoying messages on the screen, or do nothing at all. In any case, viruses are undesirable for computer users, regardless of their nature [12]. Modern viruses also take advantage of the always-connected Internet to spread on a global level. Therefore, early detection of viruses is necessary to minimize potential damage.

There are many antivirus defense mechanisms available today. The most widely used mechanism is signature detection, which detects viruses by searching the files on a computer system and looking for known binary strings — or other signatures — of viral files [1]. Another mechanism for virus detection is code emulation, which creates a virtual machine to execute suspicious programs and monitor unusual activities.

To evade signature detection, virus writers have adopted code obfuscation techniques to create highly metamorphic computer viruses. Since metamorphic viruses use various code obfuscation techniques to change their appearance from generation to generation, signature-based scanners might not be able to detect all generations of such viruses.

In order to combat metamorphic viruses, virus detection tools based on statistical analysis have been studied. A tool based on the Hidden Markov Model (HMM) was developed in [2], and the 
results are encouraging. In [2], it was shown that metamorphic viruses created by a welldesigned metamorphic engine could be detected using statistical analysis tools based on HMMs.

The goal of this project is to develop a standalone metamorphic engine to show that it is possible to defeat HMM-based detection tools developed in [2]. We employ code obfuscation techniques, including equivalent instruction substitution, dead code insertion, and rearrangement of instruction order. In addition, we have designed our metamorphic engine to generate highly discrete copies of the base virus. Furthermore, each discrete viral copy will randomly select a "normal" file and make itself similar to that normal file. These morphed copies have been tested against an HMM of the base virus family, normal files, and our own morphed copies. We also tested our morphed copies against commercial virus scanners.

This paper is organized as follows. In Section 2, we provide background information on computer viruses and discuss some possible defenses. Section 3 describes a similarity test that is useful for quantifying the degree of metamorphism, as well as describes HMMs and their use in detecting metamorphic viruses. Section 4 details the design and implementation of our metamorphic generator. Section 5 outlines the experimental results for our metamorphic virus engine. In Section 6, we consider a detection technique for identifying viruses generated by our engine. Section 7 presents our conclusions. Finally, we discuss possible extensions to the project and future work in Section 8.

\section{Computer Virus Evolution and Detection}

A computer virus is a small piece of software that piggybacks on real programs [24]. For example, a virus can insert itself into a spreadsheet program. When a user opens the spreadsheet and executes the program, the virus also runs, and it has the chance to reproduce (by attaching to other programs) and wreak havoc [24]. 


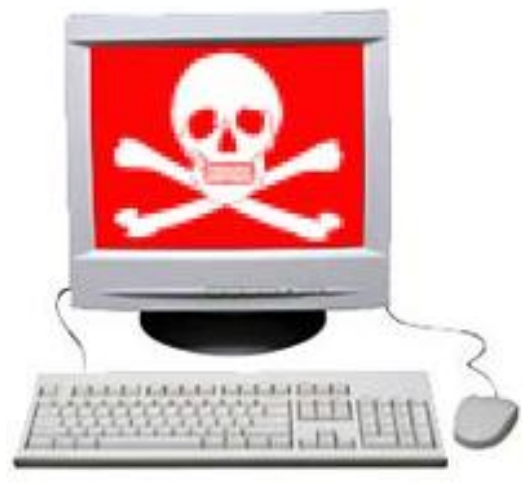

Figure 1. A virus can spell doom for your computer [24]

Viruses usually have an "infect" phase and an "attack" phase. When an embedded virus runs during the infect phase, it will try to infect other executables by copying itself into them. Viruses that do not have attack phases are considered harmless. These viruses just replicate themselves and generally do not impact the normal system operation. However, most viruses also have a destructive attack phase in which they do considerable damage. These viruses usually activate their attack phase based on some sort of event. During the attack phase, viruses will reveal themselves in tangible ways - by doing anything from displaying silly messages to destroying all of the computer's data. The trigger event might be a date, the number of times the virus has been replicated, or something similar [24].

\subsection{Antivirus Defense Techniques}

Techniques for generating viruses have advanced over time, as have the anti-virus techniques for detecting such advanced viruses. In this section, we will outline some of the popular antivirus techniques.

\subsubsection{Signature Detection}

Signature detection is the earliest anti-virus technique and is still the most widely used technique today [4]. In general, a signature of a virus is a string of bits found in a virus, but not in other executables [17]. When a new virus is analyzed, its signature will be put into the virus scanner database. During the scanning process, a signature-based virus detection tool will search all of the files in a system for known signatures. It will flag the file as infected if a known virus signature is found. For example, when an executable file is infected by the W32/Beast virus, it 
will contain a binary signature of "83EB 0274 EB0E 740A 81EB $03010000 ”[13]$. The virus scanner searches executables for this signature. If this signature is present in any executable file, it is declared to be the Beast virus.

\subsubsection{Heuristic Analysis}

Heuristic analysis [23] is a method designed to detect previously unknown computer viruses, as well as new variants of viruses already in the wild. Heuristic analysis detects viruses by executing questionable programs or scripts in a virtual machine and monitoring them for common viral activities, such as replication, overwriting files, and attempts to hide. If such actions are detected, the suspicious programs will be flagged as viruses and will raise alerts.

Another method of heuristic analysis is to decompile the viral program, then analyze the code. This type of heuristic analysis then looks for instructions that are commonly found in viral programs. If the source code contains a certain percentage of instructions that match common viral instructions, the file is flagged and users are alerted.

The effectiveness of heuristic analysis is fairly low due to the number of false positives. The reason for this is that heuristic analysis mostly operates on the basis of past experience [23]; it might miss new viruses that contain codes not found in any previously known viruses. However, heuristic analysis is also evolving in terms of its ability to reveal new viruses, so it still provides some measure of detection.

\subsection{Virus Evolution}

\subsubsection{Virus Obfuscation Techniques}

Virus-like programs first appeared on microcomputers in the 1980s [19]. Since then, the battle between anti-virus (AV) researchers and virus writers continues. Virus writers constantly develop new obfuscation techniques to make virus code more difficult to detect [19]. To escape from generic scanning, a virus can modify its code and alter its appearance on each infection. The techniques that have been employed to achieve this end range from encryption, to polymorphic techniques, to modern metamorphic techniques [20]. 


\subsubsection{Encrypted Viruses}

The simplest method to hide the virus body is to encrypt it with different encryption keys. A virus generated by this method usually consists of a small decrypting engine (a decryptor) and the encrypted body. When executed, the virus will first decrypt itself and then create another copy by encrypting itself with a different encryption key. Since a different encryption key is used for each infection, the virus body will look different as well. Efficient crypto methods, such as XOR, the key with the virus body, are typically used for this type of virus [2]. Although the virus body looks different from generation to generation, the decryptor that is embedded in the virus remains constant for all generations. As a result, it is possible to detect the virus indirectly by recognizing the code pattern of the decryptor.

\subsubsection{Polymorphic Viruses}

Polymorphism is a more sophisticated technique that virus writers implement to overcome the weakness of encrypted viruses. Polymorphic viruses hide the decryptor code by mutating it. This makes the decryptor code appear to be different from generation to generation. In addition, polymorphic viruses have the capability of generating a large number of unique decryptors that use different encryption methods to encrypt virus bodies [2]. Therefore, polymorphic viruses lack unique patterns on each infection.

Although a polymorphic virus lacks a unique pattern on each infection, it is still possible to detect the actual virus code through the code emulation technique. To detect polymorphic viruses, anti-virus software incorporates a code emulator that allows the viruses to run within the emulation environment and dynamically detects the decrypted virus bodies.

\subsubsection{Metamorphic Viruses}

Instead of trying to hide the decryptor, as in polymorphic viruses, virus writers have developed advanced metamorphic techniques to change the actual virus code for each infection $[15,18]$. According to Muttik [14], "Metamorphics are bodypolymorphics." Since the virus body already has different appearances, encryption is no longer needed for hiding the virus. Different generations of a metamorphic virus can have different "shapes" while maintaining the virus' 
original behavior. Figure 2 shows the diagrammatical illustration by Szor in [20]. In this section, we will discuss some of the metamorphic techniques employed by metamorphic virus writers. In general, metamorphic virus generators incorporate more than one of these techniques in order to produce highly morphed metamorphic viruses.

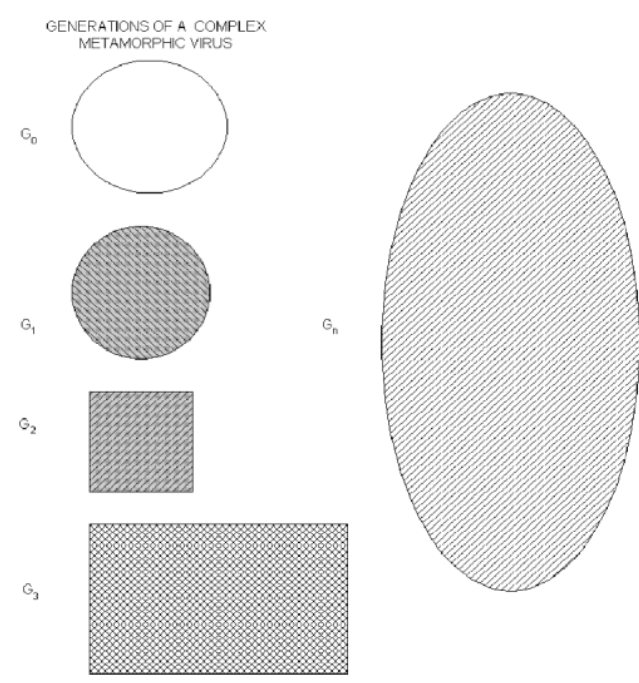

Figure 2. Multiple shapes of a metamorphic virus body [20]

\subsubsection{Register Swap}

Register swap is the simplest metamorphic technique. It mutates the virus body by swapping the operand registers with different registers. For example, instruction "pop edx" might be replaced with "pop eax." The W95/Regswap virus [7] is among the early metamorphic viruses that use register swap technique. With this technique, the opcode sequence remains unchanged, as shown in Figure 3. Such viruses can usually be detected by a wildcard string [19]. 


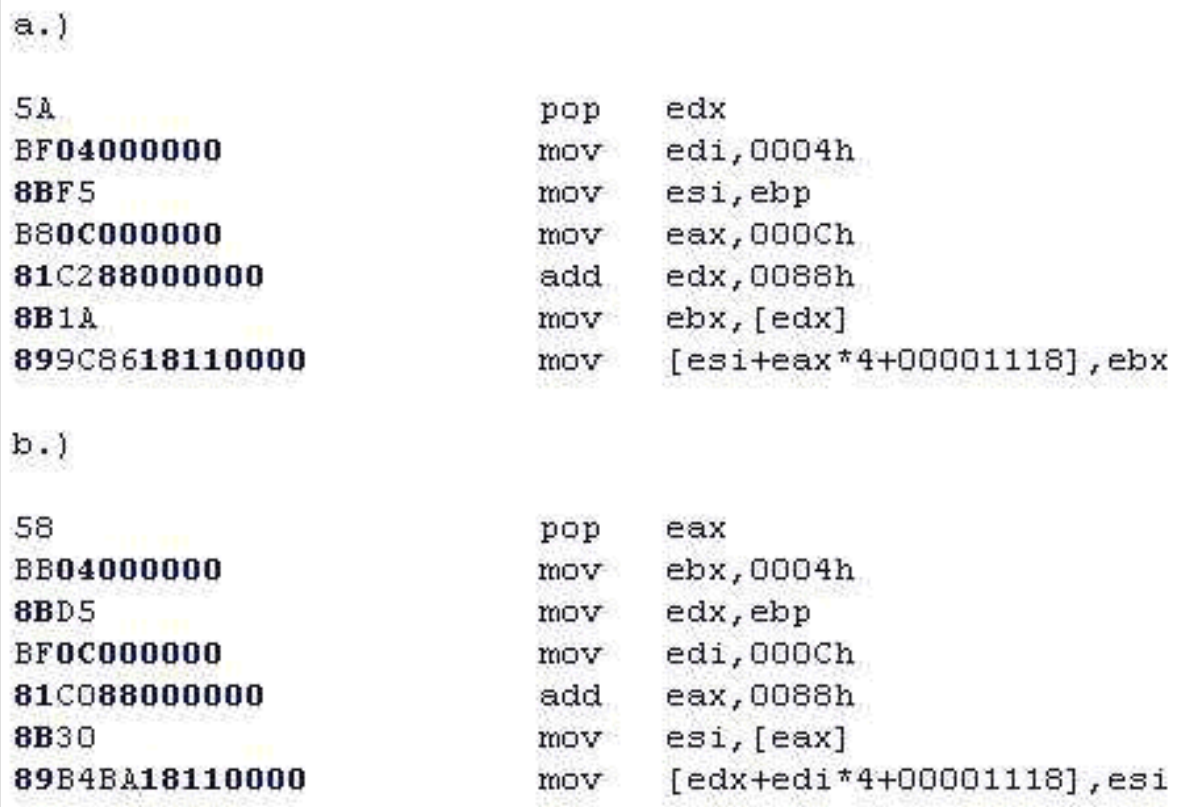

Figure 3. Two different generations of RegSwap [9]

\subsubsection{Subroutine Permutation}

Subroutine permutation technique changes the appearances of a virus by reordering the virus' subroutines. If a virus has $\mathrm{n}$ different subroutines, then it can generate $\mathrm{n}$ ! different generations without repeating. The W32/Ghost virus [19] is one of the viruses that incorporates the subroutine permutation technique. This particular virus has 10 subroutines. Therefore, it can generate 10 ! (or $3,628,800$ ) unique copies. However, the virus may still be detected with search strings [19], as the content of each subroutine remains constant. 


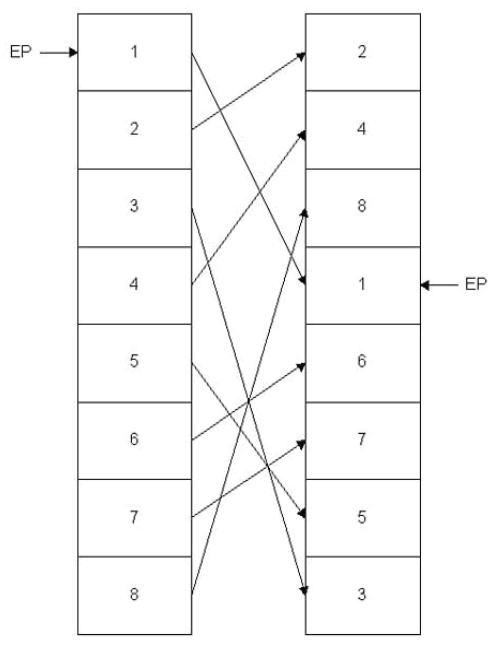

Figure 4. Subroutine permutation

\subsubsection{Garbage Instruction Insertion}

Many complex metamorphic viruses [2] incorporate the garbage instruction insertion technique due to its effectiveness. Garbage instructions are instructions that are either not executed (dead code) or have no effect (do nothing) on program outcomes [13]. By inserting garbage instructions between core instructions randomly, a virus can potentially generate infinite unique copies.

Examples of "do nothing" instructions are "nop," "add R 0", or "sub R 0". [6]. A complete list of "do nothing" instructions can be found in Appendix C. Dead code instructions are usually generated by inserting "jump" instructions to point to the next actual instructions. Any instructions between the "jump" instructions and the next actual instructions will never be executed. The Win95/Zperm virus is one of the viruses that incorporates this technique. As illustrated in Figure 5 [19], the Win95/Zperm family of viruses creates new mutations by reordering core instructions and inserting jump and garbage instructions. 

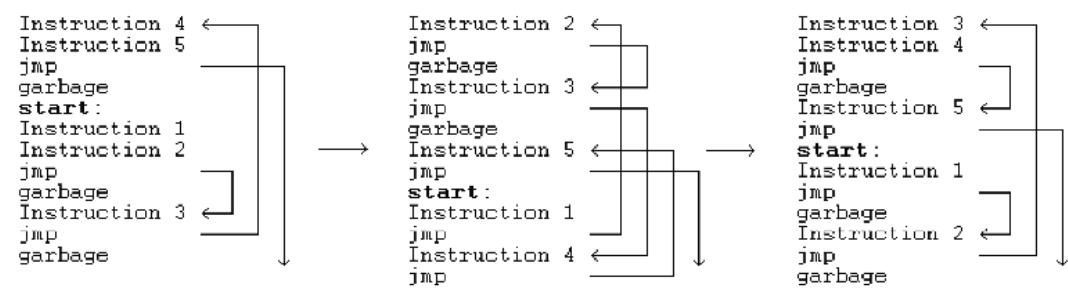

Figure 5. Zperm virus [19].

\subsubsection{Instruction Substitution}

Instruction substitution is another common technique for generating metamorphic viruses. Instruction substitution is the replacement of an instruction or a group of instructions with an equivalent instruction or group [15]. For example, "inc eax" is equivalent to "add eax, 1," and "move eax, edx" can be replaced by "push edx" followed by "pop eax." The W32/MetaPhor virus is one of the metamorphic virus generators that incorporates the instruction substitution technique. Some examples of instruction substitution used by the W32/MetaPhor virus [19] are presented in Table 1.

\begin{tabular}{|ll|ll|}
\hline \multicolumn{2}{|l|}{ Single Instruction } & Instruction block \\
\hline \hline XOR & Reg, Reg & MOV & Reg, O \\
\hline MOV & Reg, Imm & PUSH & Imm \\
& POP & Reg \\
\hline \multirow{2}{*}{ OP } & Reg, Reg2 & MOV & Mem, Reg \\
& & MOV & Mem, Reg2 \\
\hline
\end{tabular}

Table 1. Examples of instruction substitution used by W32/MetaPhor virus [19]

\subsubsection{Transposition}

Transposition is the reordering of the instruction execution sequence. This can only be done if the affected instructions have no dependency between them. For example, if the second instruction does not depend on the result of the first instruction, then the execution order of these two instructions can be swapped. Consider the following example from [24]:

op1 [r1] [, r2]

op2 [r3] [, r4]; here r1 and/or r3 are to be modified

We can swap the above two instructions only if: 
1. r1 not equal to $\mathrm{r} 4$; and

2. r2 not equal to $\mathrm{r} 3$; and

3. r1 not equal to $r 3$.

\subsubsection{Formal Grammar Mutation}

Formal grammar mutation is the formalization of existing code mutation techniques widely used in viruses (polymorphism and metamorphism) by means of formal grammars and automatons [10]. In general, classic metamorphic generators can be presented as bulky, non-deterministic automata, because all possible input characters are specified for each state of automata [10]. By formalizing existing code mutation techniques into formal grammar, one can then apply formal grammar rules to create new viral copies with great variations.

A simple polymorphic decryptor code, as shown in Figure 6, can generate a new viral copy (Figure 7) that looks very different than the original copy.

$$
\begin{array}{ll}
\text { 1. } & \text { mov } R_{1} \text {, len } \\
\text { 2. } & \text { mov } R_{2}, \text { beg } \\
\text { 3. } & \text { xor }\left[R_{2}\right] \text {, key } \\
\text { 4. } & \text { add } R_{2}, 4 \\
\text { 5. } & \text { sub } R_{1}, 4 \\
\text { 6. } & \text { jnz step_3 }
\end{array}
$$

\begin{tabular}{|c|c|}
\hline OO PUSH 44554433 & 00 XOR EDI,EDI \\
\hline 01 POP ESI & 01 LEA EDI, [EDI+124] \\
\hline 02 SUB EBX, EBX & 02 PUSH 44554433 \\
\hline 03 ADD EBX, 124 & 03 POP ESI \\
\hline $04 \mathrm{XOR}$ [ESI], d20b9a65 & 04 MOV EDX, [ESI] \\
\hline 05 ADD ESI, 4 & 05 NOT EDX \\
\hline 06 SUB EBX, 4 & 06 AND EDX,d75d40bc \\
\hline $07 \mathrm{JZ} \$+2$ & 07 AND [ESI], 28a2bf43 \\
\hline \multirow[t]{5}{*}{$08 \mathrm{JMP} \$+f 0$} & $08 \mathrm{OR}$ [ESI], EDX \\
\hline & 09 ADD ESI, 4 \\
\hline & 10 SUB EDI, 4 \\
\hline & $11 \mathrm{JZ} \$+2$ \\
\hline & $12 \mathrm{JMP} \$+\mathrm{e} 4$ \\
\hline
\end{tabular}

Figure 6. Simple polymorphic decryptor

Figure 7. Generated polymorphic decryptor 


\section{Similarity and the HMM}

This section outlines the similarity test and the HMM developed in [2] for detecting metamorphic viruses.

\subsection{Similarity Test}

Metamorphism is, arguably, the best approach to escape detection. Different generations of a virus must look different in order to avoid detection by signature-based scanning. Some of the virus creation toolkits come with the ability to generate morphed versions of the same virus, even from identical configurations. The similarity test previously studied [2] has shown that it is suitable to measure the effectiveness of a metamorphic virus generator. In this section, we outline the steps of the similarity test and the meaning of its result.

\subsubsection{Similarity Test Method}

The similarity test employed the method developed by Mishra in [11]. It compares two assembly programs and assigns a quantitative score to represent the percentage of similarity between the two programs. Mishra's method is outlined below and is illustrated graphically in Figure 8.

1) Given two assembly programs, $X$ and $Y$, first extract the sequence of opcodes for each of the programs, excluding comments, blank lines, labels, and other directives. The result is two opcode sequences of length $\mathrm{n}$ and $\mathrm{m}$, where $\mathrm{n}$ and $\mathrm{m}$ are the numbers of opcodes in programs $\mathrm{X}$ and $\mathrm{Y}$, respectively. Each opcode is assigned an opcode number: the first opcode is 1 , the second is 2 , and so on.

2) Compare the two opcode sequences by considering all subsequences of three consecutive opcodes from each sequence. Then count as a match any case where all three opcodes are the same in any order. A mark will be placed on a graph coordinate $(x, y)$ of the match, where $\mathrm{x}$ is the opcode number of the first opcode of the three-opcode subsequence in program $\mathrm{X}$, and $\mathrm{y}$ is the opcode number of the opcode subsequence in program $\mathrm{Y}$. 
3) After comparing the entire opcode sequences and marking all the match coordinates, a graph plotted on a grid of dimension $\mathrm{n} \times \mathrm{m}$ is obtained. Opcode numbers of program $\mathrm{X}$ are represented on the $\mathrm{x}$-axis and those of program $\mathrm{Y}$ are represented on the $\mathrm{y}$-axis. To remove noise and random matches, only those line segments of length greater than some threshold values (e.g., five) will be retained.

4) Since the test is basically a sequential match between the two opcode sequences, identical segments of opcodes will form line segments parallel to the main diagonal (if $n=m$, the main diagonal is simply the 45 degree line). If a line segment falls right on the diagonal, the matching opcodes are at identical locations on the two opcode sequences. A line off the diagonal indicates that the matching opcodes appear at different locations in the two files.

5) For each axis, the sum of the number of opcodes that are covered by one or more of the matching line segments is calculated. This number is divided by the respective total number of opcodes ( $\mathrm{n}$ for program $\mathrm{X}$ and $\mathrm{m}$ for program $\mathrm{Y}$ ) to give the percentage of opcodes that match some opcodes in the other program. The similarity score for the two programs is the average of these two percentages.

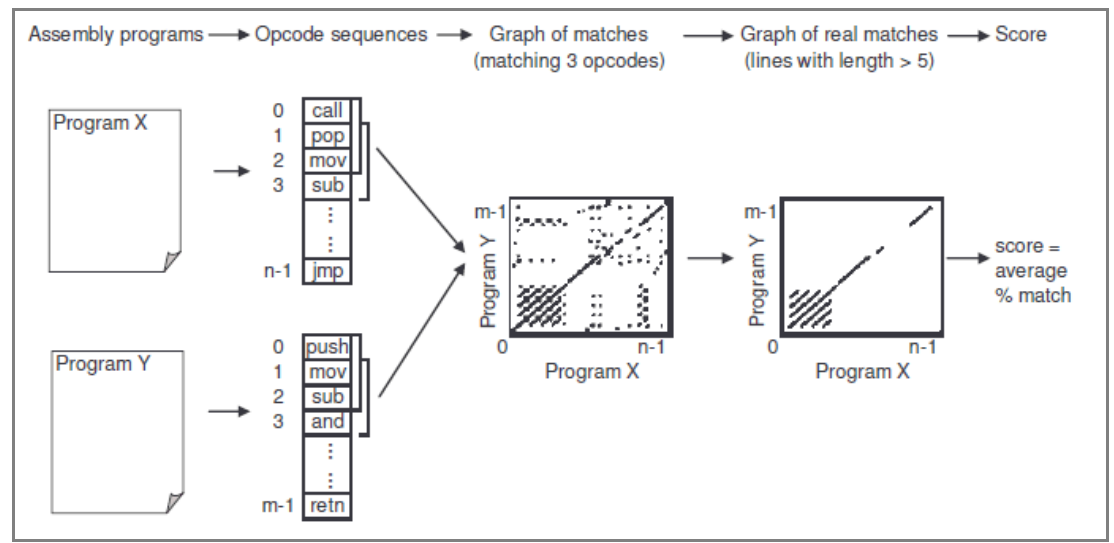

Figure 8. Process of finding the similarity between two assembly programs [2]

\subsubsection{Similarity Test Results}

Upon the completion of the similarity test, a graph will be generated to visualize the similarity results of the assembly files. Usually, a graph generated by plotting all of the matches for file $\mathrm{X}$ and $\mathrm{Y}$ (see Figure 9-a) is very populated. This makes it difficult to understand the similarity result. A cleaner graph can be generated by dropping all of the matches that are less than a 
specified threshold. Figure 9-b shows a graph that is generated with threshold of 5. The latter provides a clearer visualization of the similarity result. Previous studies in [2] have shown that the best metamorphic engine (NGVCK) achieves a similarity score of about $10 \%$, the lowest similarity score recorded, whereas normal files usually have a similarity score of about $30 \%$.

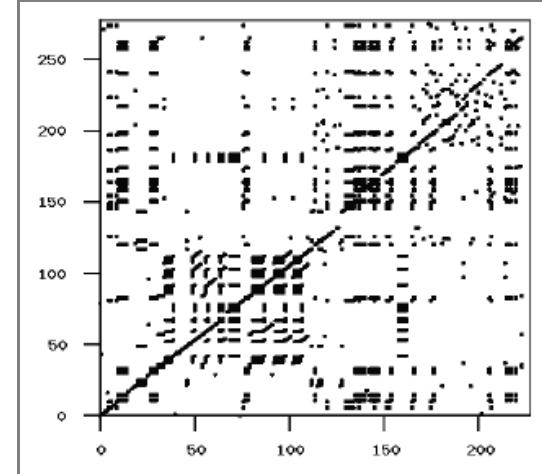

(a) All matches

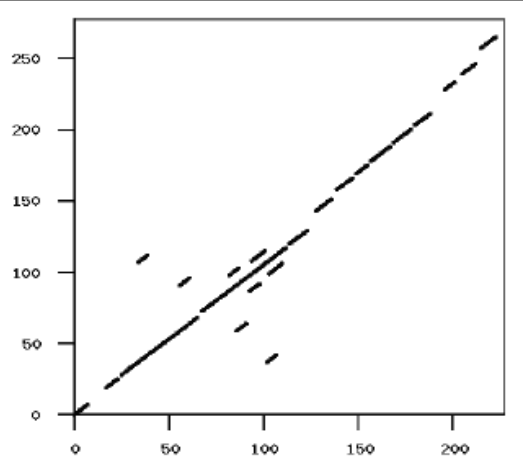

(b) With threshold

Figure 9. Similarity graph [2]

\subsection{HMM}

The Hidden Markov Model (HMM) is a statistical pattern analysis algorithm. The notations used in the HMM are as follows:

$$
\begin{aligned}
& T=\text { Length of the observed sequence } \\
& N=\text { Number of states in the model } \\
& M=\text { number of distinct observation symbols } \\
& O=\text { Observation sequence }\left\{00,01, \ldots, O_{T-1}\right\} \\
& A=\text { State transition probability matrix } \\
& B=\text { Observation probability distribution matrix } \\
& \pi=\text { Initial state distribution matrix }
\end{aligned}
$$

A generic Hidden Markov Model is illustrated in Figure 10. The state and observation at time $\mathrm{t}$ are represented by $\mathrm{X}_{\mathrm{t}}$ and $\mathrm{O}_{\mathrm{t}}$ respectively. The Markov process - which is hidden behind the dashed line - is determined by the initial state $\mathrm{X}_{0}$ and the A matrix. Only the $\mathrm{O}_{\mathrm{i}}$ is observable, which is related to the actual states of the Markov process by the matrices B and A. 


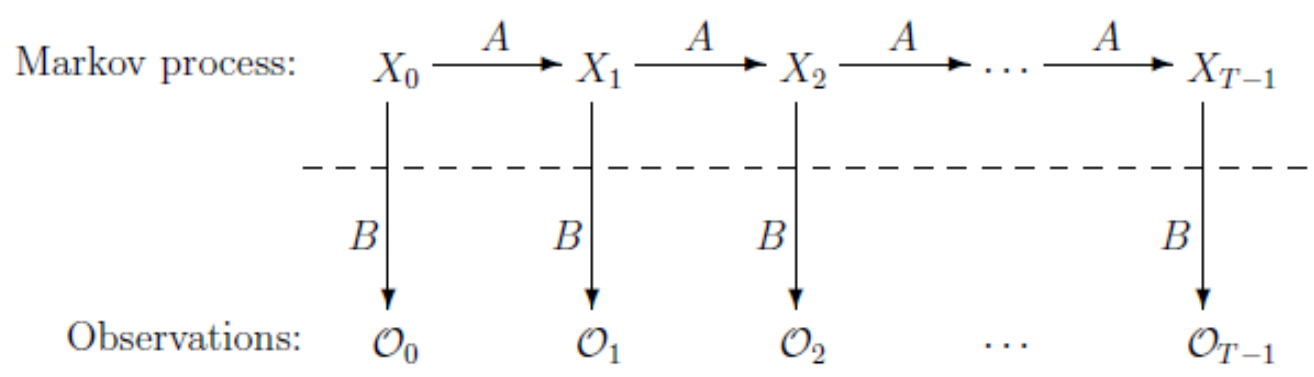

Figure 10. Generic Hidden Markov Model [14]

HMMs are widely used for protein modeling and speech recognition applications [3]. In general, an HMM first creates a training model that represents the input data (training data). The training model contains a list of unique symbols observed in the input data and their positions in the input sequence. This model will be used by the HMM to determine if a given new input sequence has a pattern similar to that of the model.

Recently, HM Ms have been successfully used to detect metamorphic viruses [2, 9]. Although metamorphic engines use varies code obfuscation techniques to change the appearance of viral copies, some similar patterns exist within the same family of viruses. An HMM collects the input data from all known viruses and builds the training models (one for each family virus) based on these input data. Subsequently, any file can be tested against these models to determine if it belongs to one of them. If an input file belongs to a model, then it is a member of the virus family that the model represents.

\subsubsection{HMM Example}

A simple example in [14] illustrates the inner working of an HMM. Suppose that one has no prior knowledge of the average annual temperature for any given year and wants to determine this information based on the observation of tree sizes (S-small, M-medium, L-large). To keep the example simple, let us assume that the annual temperature can be either hot $(\mathrm{H})$ or cold $(\mathrm{C})$. In addition, we know the probability of the annual temperature trend: a hot year followed by another hot year $(\mathrm{HH})$ is 0.7 ; a hot year followed by a cold year $(\mathrm{HC})$ is 0.3 ; a cold year followed by a hot year is 0.4 ; and a cold year followed by another cold year is 0.6 . Figure 11 shows the matrix representation of these probabilities. 


$$
\left.\begin{array}{c}
H \\
C \\
C \\
0.7
\end{array}\right]
$$

\section{Figure 11. Temperature transition probability}

Furthermore, we know that the correlation between tree sizes and temperature is as follows:

- In a hot year, the probability of a tree being small is 0.1 , being medium 0.4 , and being large 0.5 .

- In a cold year, the probability of a tree being small is 0.7 , being medium 0.2 , and being large 0.1 .

Figure 12 shows the correlation probability between temperatures and tree sizes in a matrix representation.

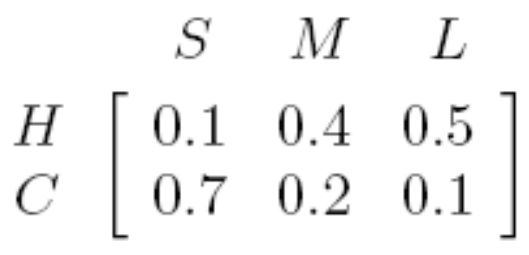

Figure 12. Tree size probability

In this example, the annual temperatures are the states, and the tree sizes are the observable symbols. The probability of different tree sizes at each temperature represents the probability of the observation symbols in each state. The states $(\mathrm{H}$ and $\mathrm{C})$ are hidden, since the temperature cannot be seen directly. We can only see the observation symbols (S, M, and L), which are statistically related to the states. With the knowledge of correlation probabilities for annual temperature and tree sizes, we can build an HMM model as shown in Figure 13. 


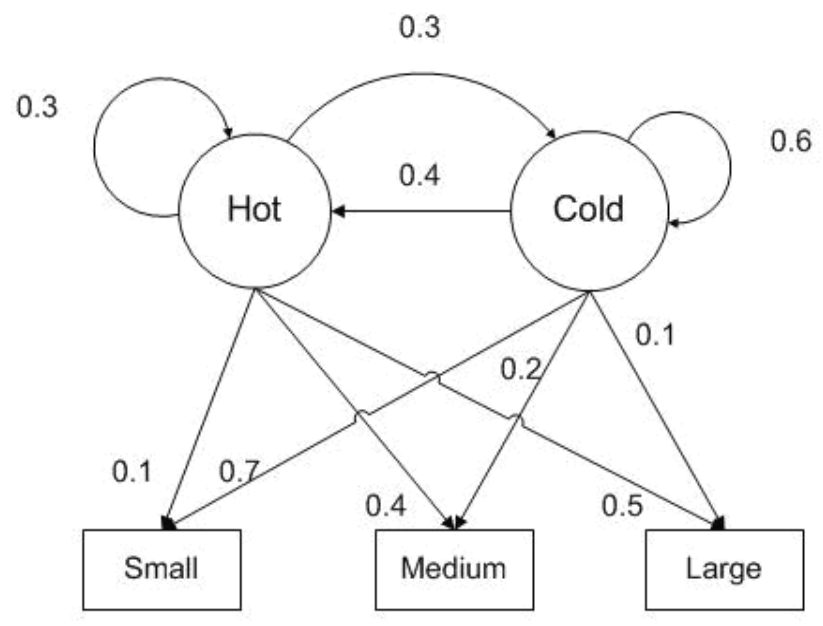

Figure 13. HMM model [14]

Suppose we have observed that the tree sizes (observation symbols) sequence for four consecutive years is (S, M, S, L). We want to use this observed sequence to find the annual temperature sequence (states).

To solve this problem with an HMM algorithm, we must first construct our HMM parameters as follows:

- State transition probability matrix

$$
A=\left[\begin{array}{ll}
0.7 & 0.3 \\
0.4 & 0.6
\end{array}\right]
$$

- The observation probability distribution matrix

$$
B=\left[\begin{array}{lll}
0.1 & 0.4 & 0.5 \\
0.7 & 0.2 & 0.1
\end{array}\right]
$$

—Number of states in the model $\mathrm{N}=2$ (hot and cold)

—Number of distinct observation symbols $\mathrm{M}=3$ (small, medium, and large)

-Given initial state distribution matrix

$$
\pi=\left[\begin{array}{ll}
0.6 & 0.4
\end{array}\right]
$$

The HMM steps used to determine the state transition for a given observation (S, M, S, L) of length $\mathrm{T}=4$ will be as follows: 
1. Determine all possible state transitions $=\mathrm{N}^{\mathrm{T}}$.

2. Calculate the probability of a given observation sequence for each state transition of step 1 (shown in Table 2). For example, calculate the probability of sequence HHCC as follows:

$$
\begin{aligned}
\mathrm{P}(\mathrm{HHCC}) & =\pi_{\mathrm{H}} * \mathrm{~b}_{\mathrm{H}}(\mathrm{S}) * \mathrm{aH}, \mathrm{H}^{*} \mathrm{~b}_{\mathrm{H}}(\mathrm{M}) * \mathrm{aH}, \mathrm{C}^{*} \mathrm{~b}_{\mathrm{C}}(\mathrm{S}) * \mathrm{ac}, \mathrm{C} * \mathrm{bc}_{\mathrm{C}}(\mathrm{L}) \\
& =(0.6) *(0.1) *(0.7) *(0.4) *(0.3) *(0.7) *(0.6) *(0.1) \\
& =0.000212
\end{aligned}
$$

3. The annual temperature sequence is the one with the highest probability. In this case, the answer would be "CCCH," since it has the highest probability.

\begin{tabular}{|c|c|}
\hline state sequence & probability \\
\hline HHHH & 0.000412 \\
HHHC & 0.000035 \\
HHCH & 0.000706 \\
HHCC & 0.000212 \\
HCHH & 0.000050 \\
HCHC & 0.000004 \\
HCCH & 0.000302 \\
HCCC & 0.000091 \\
$\mathrm{CHHH}$ & 0.001098 \\
$\mathrm{CHHC}$ & 0.000094 \\
$\mathrm{CHCH}$ & 0.001882 \\
$\mathrm{CHCC}$ & 0.000564 \\
$\mathrm{CCHH}$ & 0.000470 \\
$\mathrm{CCHC}$ & 0.000040 \\
$\mathrm{CCCH}$ & 0.002822 \\
$\mathrm{CCCC}$ & 0.000847 \\
\hline$\Sigma$ probability & 0.009629 \\
\hline max probability & 0.002822 \\
\hline
\end{tabular}

Table 2. Probabilities of observing (S, M, S, L) for all possible state sequences [14]

The above brute-force method of computing HMM results requires an exponential amount of work. This is generally infeasible. The beauty an HMM is that it includes efficient algorithms to solve the three problems in which we are interested. These are [14]:

1. Given the model $\lambda=(\mathrm{A}, \mathrm{B}, \pi)$ and an observation sequence $\mathrm{O}$, find $\mathrm{P}(\mathrm{O} \mid \lambda)$, which is the likelihood of observing the sequence $\mathrm{O}$ given the model.

2. Given the model $\lambda=(\mathrm{A}, \mathrm{B}, \pi)$, find an optimal state sequence for the underlying Markov process. That is, uncover the hidden part of the HMM. 
3. Given an observation sequence $\mathrm{O}$, the number of symbols $M$, and the number of states $\mathrm{N}$, find the model $\lambda=(\mathrm{A}, \mathrm{B}, \pi)$ that maximizes the probability of $\mathrm{O}$. In other words, the model is trained to best fit the observed data.

The fact that there are efficient algorithms for solving the three HMM problems provides a fundamental building block for constructing the HMM-based virus detector. More precisely, the HMM-based virus detector developed in [2] was implemented the following algorithms to solve the three HMM problems:

1. The Forward algorithm:

The equation to find the likelihood of an observed sequence is given as

$$
P(O, X \mid \lambda)=\pi_{x_{0}} b_{x_{0}}\left(O_{0}\right) a_{x_{0}, x_{1}} b_{x_{1}}\left(O_{1}\right) a_{x_{1}, x_{2}} \ldots a_{x_{T-2}, x_{T-1}} b_{x_{T-1}}\left(O_{T-1}\right) .
$$

The naïve approach to solve the above equation would be to generate all possible state sequences $\mathrm{X}_{\mathrm{i}}$ of length $\mathrm{T}$ and sum over the probabilities $\mathrm{P}\left(\mathrm{O}, \mathrm{X}_{\mathrm{i}} \mid \lambda\right)$ :

$$
\begin{aligned}
P(O \mid \lambda) & =\sum_{X_{i}} P\left(O, X_{i} \mid \lambda\right) \\
& =\sum_{X_{i}} \pi_{x_{0}} b_{x_{0}}\left(O_{0}\right) a_{x_{0}, x_{1}} b_{x_{1}}\left(O_{1}\right) a_{x_{1}, x_{2}} \ldots a_{x_{T-2}, x_{T-1}} b_{x_{T-1}}\left(O_{T-1}\right)
\end{aligned}
$$

However, this direct computation requires $2 \mathrm{TN}^{\mathrm{T}}$ computations.

The forward algorithm (sometimes called the $\alpha$-pass), which requires only $\mathrm{N}^{2} \mathrm{~T}$ computations, is a much more efficient algorithm for solving the above equation. Instead of computing all possible states directly, the forward algorithm inductively computes the states as follows:

For $\mathrm{t}=0,1, \ldots, \mathrm{T}-1$ and $\mathrm{i}=0,1, \ldots, \mathrm{N}-1$, define a forward variable

$$
\alpha_{t}(i)=P\left(O_{0}, O_{1}, \ldots, O_{t} x_{t}=q_{i} \mid \lambda\right)
$$

which denotes the probability of observing the partial sequence $\left(\mathrm{O}_{0}, \mathrm{O}_{1}, \ldots, \mathrm{O}_{\mathrm{t}}\right)$ up to time $t$ and being in state $\mathrm{q}_{\mathrm{i}}$ at time $\mathrm{t}$. The forward variables can then be computed recursively using the following steps:

Step 1. Initialization

$$
a_{0}(i)=\pi_{i} b_{i}\left(O_{0}\right), \quad \text { for } i=0,1, \ldots, N-1
$$


Step 2. Compute forward variables inductively

$$
\alpha_{t}(i)=\left[\sum_{j=0}^{N-1} \alpha_{t-1}(j) a_{j i}\right] b_{i}\left(O_{t}\right), \quad \text { for } t=1,2, \ldots, T-1 \text { and } i=0,1, \ldots, N-1 \text {. }
$$

The probability of $\mathrm{P}(\mathrm{O} \mid \lambda)$ can then be calculated as

$$
\begin{aligned}
P(O \mid \lambda) & =\sum_{i=0}^{N-1} P\left(O_{0}, O_{1}, \ldots, O_{T} x_{T}=q_{i} \mid \lambda\right) \\
& =\sum_{i=0}^{N-1} \alpha_{T-1}(i) .
\end{aligned}
$$

2. The Viterbi algorithm:

The Viterbi algorithm finds an optimal state sequence by finding a highest scoring overall path $X^{*}$ that maximizes the probability $\mathrm{P}(\mathrm{O}, \mathrm{X} \mid \lambda)$ as follows:

For $t=0,1, \ldots, T-1$ and $i=0,1, \ldots, N-1$, let $\delta_{t}(i)$ denote the probability of the most probable state path $\left(x_{0}, x_{1}, \ldots, x_{t}\right)$ that generates the partial sequence $\left(O_{0}, O_{1}, \ldots, O_{t}\right)$ up to time $t$ and ending in state $q_{i}$,

$$
\delta_{t}(i)=\max _{x_{0} \ldots x_{t-1}} P\left(O_{0}, O_{1}, \ldots, O_{t}, x_{0}, x_{1}, \ldots, x_{t-1}, x_{t}=q_{i} \mid \lambda\right)
$$

To find $\delta_{t}(i)$ recursively:

$$
\begin{aligned}
& \delta_{t}(i)=\max _{0 \leq j \leq N-1}\left[\delta_{t-1}(j) a_{j i}\right] b_{i}\left(O_{t}\right), \quad \text { for } t=1,2, \ldots, T-1 \text { and } i=0,1, \ldots, N-1 . \\
& \delta_{0}(i)=\pi_{i} b_{i}\left(O_{0}\right), \quad \text { for } i=0,1, \ldots, N-1
\end{aligned}
$$

Then the most likely state sequence $\mathrm{P}^{*}$ is computed as:

$$
P^{*}=\max _{0 \leq i \leq N-1}\left[\delta_{T-1}(i)\right]
$$

3. The Baum-Welch algorithm:

This algorithm provides an efficient method for adjusting the model parameters to best fit the observations. The sizes of the matrices ( $\mathrm{N}$ and $\mathrm{M})$ are fixed, but the elements of $\mathrm{A}, \mathrm{B}$, and $\pi$ are free, subject only to the row stochastic condition. The process to re-estimate the model, which is one of the most amazing aspects of HMMs, is as follows:

a. Initialize $\lambda=(\mathrm{A}, \mathrm{B}, \pi)$ with a best guess. If a best guess is not available, random values such that $\pi_{\mathrm{i}} \approx 1 / \mathrm{N}, \mathrm{a}_{\mathrm{ij}} \approx 1 / \mathrm{N}$ and $\mathrm{b}_{\mathrm{j}}(\mathrm{k}) \approx 1 / \mathrm{M}$ can be used. 
b. Compute $\alpha_{t}(i), \beta_{t}(i), \gamma_{t}(i, j)$ and $\gamma_{t}(i)$ using the following equations:

$$
\begin{gathered}
\text { For } t=0,1, \ldots, T-2 \text { and } i, j \in\{0,1, \ldots, N-1\} \text {, define "di-gammas" as } \\
\qquad \gamma_{t}(i, j)=P\left(x_{t}=q_{i}, x_{t+1}=q_{j} \mid \mathcal{O}, \lambda\right) .
\end{gathered}
$$

The di-gammas can be written in terms of $\alpha, \beta, A$, and $B$ as

$$
\gamma_{t}(i, j)=\frac{\alpha_{t}(i) a_{i j} b_{j}\left(\mathcal{O}_{t+1}\right) \beta_{t+1}(j)}{P(\mathcal{O} \mid \lambda)}
$$

The $\gamma_{t}(i)$ and $\gamma_{t}(i, j)$ are related by:

$$
\gamma_{t}(i)=\sum_{j=0}^{N-1} \gamma_{t}(i, j)
$$

c. Re-estimate the model $\lambda=(\mathrm{A}, \mathrm{B}, \pi)$ as follows:

For $i=0,1, \ldots, N-1$, let

$$
\pi_{i}=\gamma_{0}(i)
$$

For $i=0,1, \ldots, N-1$ and $j=0,1, \ldots, N-1$, compute

$$
a_{i j}=\sum_{t=0}^{T-2} \gamma_{t}(i, j) / \sum_{t=0}^{T-2} \gamma_{t}(i) .
$$

For $j=0,1, \ldots, N-1$ and $k=0,1, \ldots, M-1$, compute

$$
b_{j}(k)=\sum_{\substack{t \in\{0,1, \ldots, T-2\} \\ \mathcal{O}_{t}=k}} \gamma_{t}(j) / \sum_{t=0}^{T-2} \gamma_{t}(j) .
$$

d. If $\mathrm{P}(\mathrm{O} \mid \lambda)$ increases, go to step $\mathrm{b}$.

\subsubsection{HMM as a Virus Detection Tool}

HMM as virus detection tool requires training data to produce a model. The goal is to train one or more HMMs to represent the statistical properties of the virus family. These trained models can then be used to determine whether a given program is similar to the 
viruses in the training set. Each model is trained by collecting training data from files generated by the same generator. This also means that the resulting model is specific to the generator from which the training data originate.

To produce a training model, a set of virus files generated by the same generator must first be converted to assembly files using IDA Pro [22]. Unique assembly opcodes found in these files will constitute the HMM symbols. A long observation sequence is formed by concatenating all of the virus files within the same family. Given unique symbols and a unique observation sequence, an HMM training model can then be constructed using the solution of the third HMM problem discussed above. For example, given training data as shown in Figure 14, an HMM model can then be constructed as shown in Figure 15.

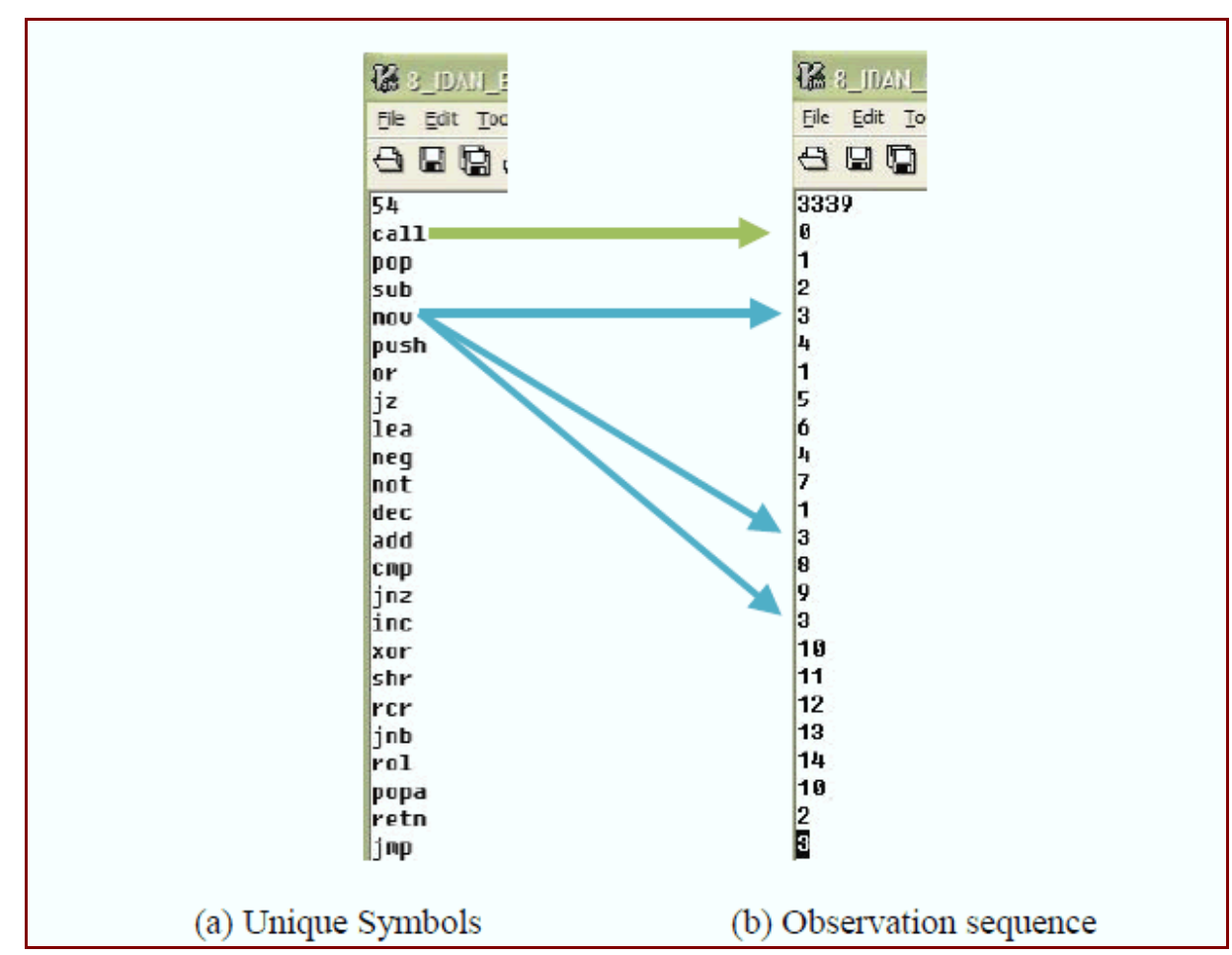

Figure 14. Training data example [5] 


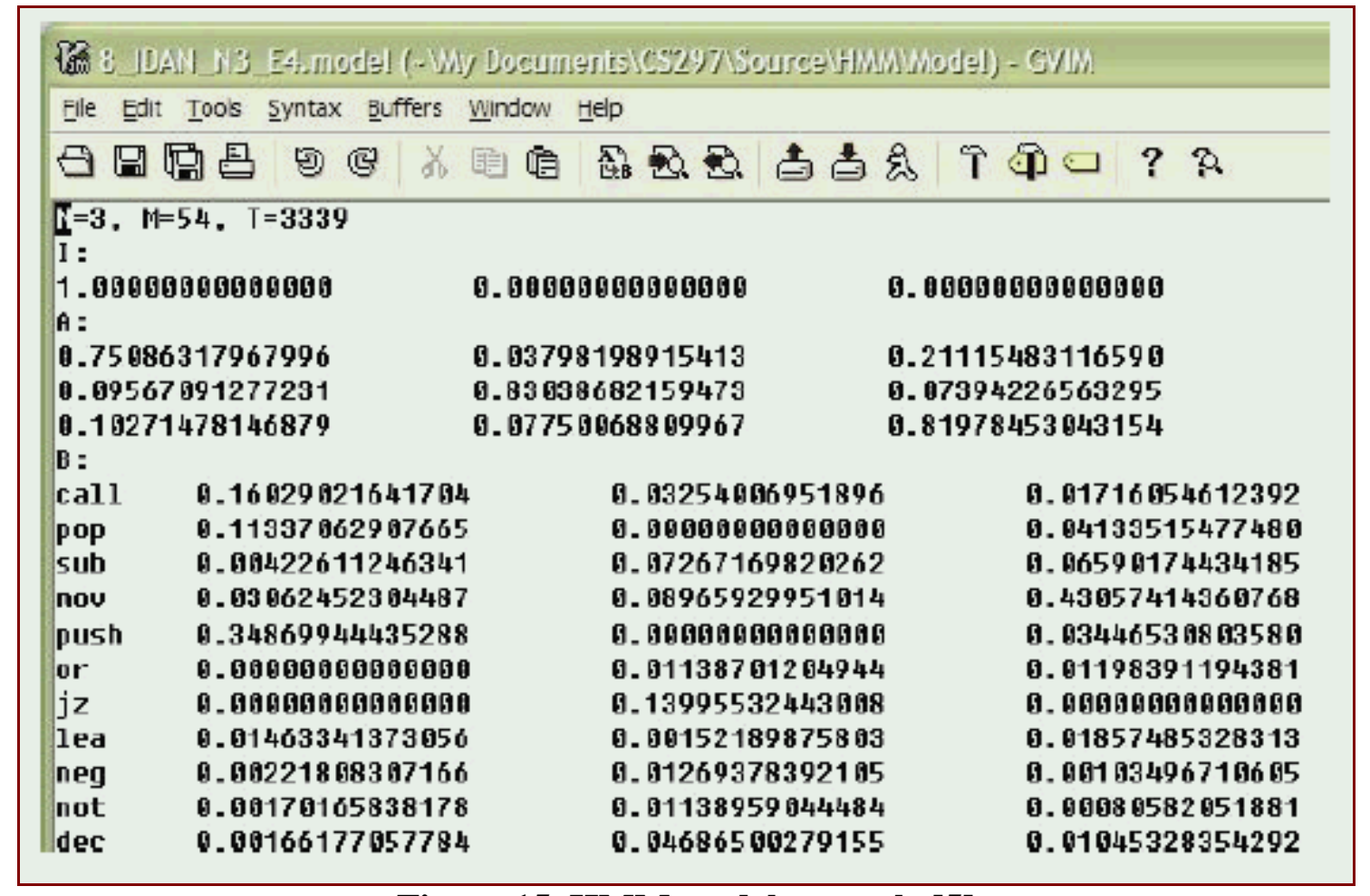

Figure 15. HMM model example [5]

After the HMM model is produced for a virus family, it can then be used to test any file to determine if it belongs to the same family. If a file has a score greater than a certain threshold, then it belongs to the same family. Otherwise, it is not in this family. An example of HMM output is shown in Figure 16. With this particular example, IDAN0 to IDAN4 are in the same virus family as the HMM model. IDAR0 to IDAR4 are not in the same virus family. 


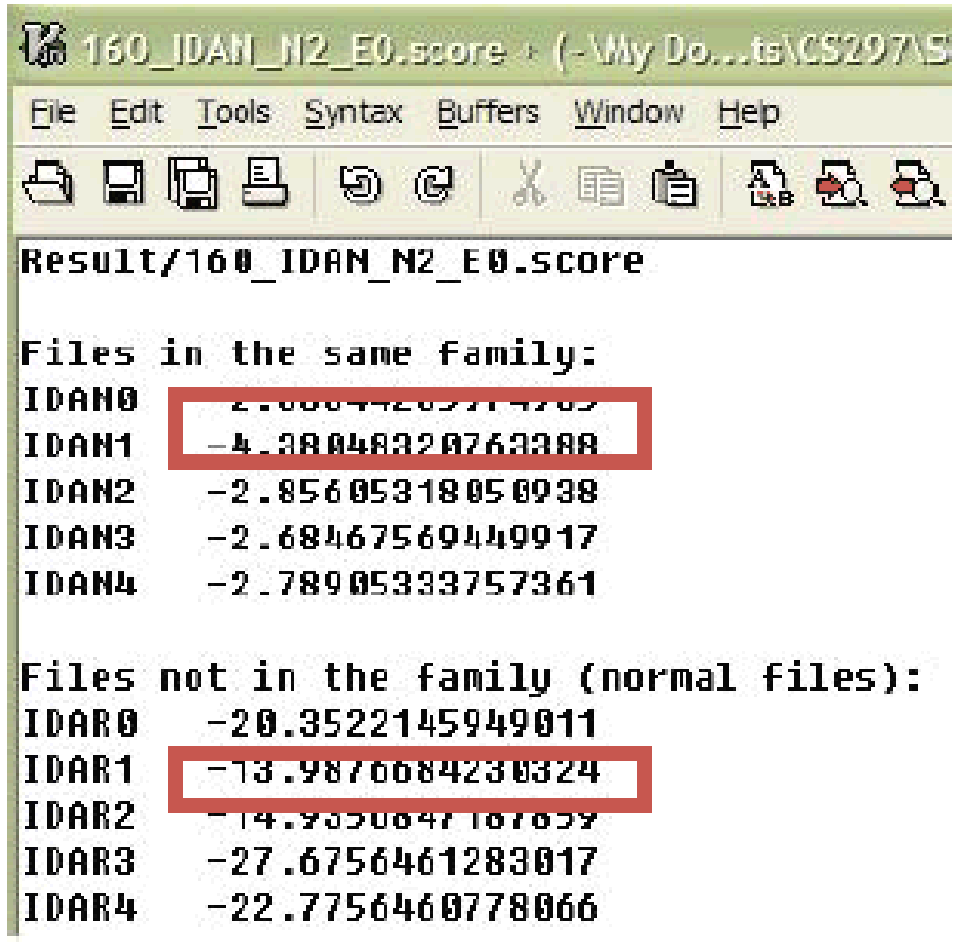

Figure 16. HMM result example [5] 


\section{Implementation}

\subsection{Introduction}

In order to produce viral copies that are hard to detect, a metamorphic engine needs to implement many code obfuscation techniques. Each implementation may have its own process to decide when and how to apply the techniques.

Even though a metamorphic engine implements all of the code obfuscation techniques, the HMM detector developed in [2] is still able to recognize the generated viruses and classify them into the same virus family. An unsuccessful attempt to escape from the HMM-based detector created in [5] has also shown that the HMM is very effective in detecting highly morphed viruses. In this project, we will develop another metamorphic engine to try to evade the HMM virus detector.

\subsection{Goal}

Our implementation was geared toward achieving the following goals:

- Generate morphed copies of a single input virus. These morphed copies should have a similarity of approximately $30 \%$ (match scores of normal files) with the base virus and among themselves.

- The morphed copies should have the same functionality as the base virus.

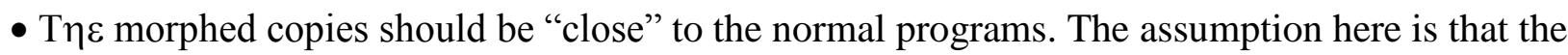
normal programs are the cygwin utility files of the same size as the base virus. The reason for using cygwin utility files is that they are probably engaged in the same low-level operations as viruses. A morphed virus is "close" to a normal program if its statistics, such as its opcode counts and opcode sequences, are more like normal files than those of an un-morphed virus. The notion of "close" will be discussed in greater detail in the next section.

- The metamorphic engine should work on any assembly program.

- Generated viral copies should be capable of escaping HMM detection. 


\subsection{Code Obfuscation Techniques Used}

Our metamorphic engine leverages the code obfuscation techniques implemented in previous work [5]. However, viruses generated by the engine developed in [5] are still detectable by the HMM detector. We have analyzed the previous engine's failure. We suspect that the reason for the failure is due to the fact that the engine applies code obfuscation techniques randomly. This randomness does not make the virus more like a normal program. Therefore, in our engine, we will apply the result of a code obfuscation operation only if it makes the virus more like a normal program. A scoring algorithm, namely the Dynamic Scoring Algorithm, has been developed for comparing the resulting virus code against the normal program code after each morph operation.

\subsubsection{Dynamic Scoring Algorithm}

To make a virus similar to a normal file, we developed an algorithm to calculate the score of similarity between the two files. The lower the score, the better the match. Since this algorithm will need to run each time we try to change an instruction, it must be as efficient as possible. The Dynamic Scoring Algorithm developed in this project need not compute the entire file each time. Instead, it only needs to compute the modified opcodes each time.

\subsubsection{Algorithm Initialization}

To initialize the dynamic scoring algorithm, two files will be passed into it as parameters. The first one is a virus, and the second one is a normal file.

Algorithm initialization will generate four master lists: individual opcode counts of the virus file; opcode-pair counts of the virus file; individual opcode counts of the normal file; and opcode-pair counts of the normal file. For example, given two short files with five opcodes, as shown in Table 3, the initializing of the algorithm will generate four lists, as shown in Table 4.

\begin{tabular}{|l|l|}
\hline Virus opcode & Normal file opcode \\
\hline Mov & Mov \\
Add & Mov \\
Mov & Sub \\
Pop & Pop
\end{tabular}


\begin{tabular}{|l|l|} 
Retn & Retn \\
\hline
\end{tabular}

Table 3. Opcode sequences of virus file and normal files

\begin{tabular}{|l|l|l|l|l|l|}
\hline $\begin{array}{l}\text { Virus opcode } \\
\text { count list }\end{array}$ & $\begin{array}{l}\text { Normal file } \\
\text { opcode count } \\
\text { list }\end{array}$ & difference & $\begin{array}{l}\text { Virus opcode-pair } \\
\text { count list }\end{array}$ & $\begin{array}{l}\text { Normal file } \\
\text { opcode-pair count } \\
\text { list }\end{array}$ & difference \\
\hline Mov (2) & Mov (2) & 0 & Mov_add (1) & Mov_add (0) & 1 \\
Add (1) & Add (0) & 1 & Add_mov(1) & Add_mov(0) & 1 \\
Pop (1) & Pop(1) & 0 & Mov_pop(1) & Mov_pop(0) & 1 \\
Retn(1) & Retn(1) & 0 & Pop_retn(1) & Pop_retn(1) & 0 \\
Sub (0) & Sub (1) & 1 & Mov_mov(0) & Mov_mov(1) & 1 \\
& & & $\begin{array}{l}\text { Mov_sub(0) } \\
\text { Sub_pop(0) }\end{array}$ & Mov_sub(1) & 1 \\
\hline
\end{tabular}

Table 4. Opcode and opcode-pair counts lists

We also compute the initial score by summing the difference of each opcode and opcode-pair counts between the two files. In the above example, the initial score will be computed as 8 .

\subsubsection{Scoring the Changes}

To check if a change will yield a better score, we only need to compute the score change by the opcode sequence change. It will take the old sequence and the new sequence as input and generate a score. A score less than 0 means the new sequence makes the two files closer to each other. A score greater than 0 means the new sequence makes the two files less similar to each other. A score of 0 means no change. This method only computes the score and does not make any changes to the master lists. For example, when we transpose "add, mov" to "mov, add," we will pass the original subsequence and the new subsequence that includes one opcode before and one opcode after plus the change itself. In this example, the two subsequences pass to the scoring changes method will be "mov, add, mov, pop" (original subsequence) and "mov, mov, add, pop" (new subsequence).

We then compute the changes in scores as follows:

1. Compute and save the to-be-affected counts.

2. Subtract the counts of the original subsequence from the master lists.

3. Add the counts of the new subsequence to the master lists.

4. Compute the affected counts against the normal file. 
Table 5 shows the result of computing the original to-be-affected score ( 5 in this case).

\begin{tabular}{|c|c|c|c|c|c|}
\hline $\begin{array}{l}\text { To-be-affected } \\
\text { Virus opcode } \\
\text { count list }\end{array}$ & $\begin{array}{l}\text { Normal file } \\
\text { opcode count } \\
\text { list }\end{array}$ & $\begin{array}{l}\text { Difference } \\
\text { before } \\
\text { changes }\end{array}$ & $\begin{array}{l}\text { To-be-affected } \\
\text { Virus opcode-pair } \\
\text { count list }\end{array}$ & $\begin{array}{l}\text { Normal file } \\
\text { opcode-pair count } \\
\text { list }\end{array}$ & $\begin{array}{l}\text { Difference } \\
\text { before } \\
\text { changes }\end{array}$ \\
\hline Mov (2) & Mov (2) & 0 & Mov_add (1) & Mov_add (0) & 1 \\
\hline Add (1) & Add (0) & 1 & Add_mov(1) & Add_mov(0) & 1 \\
\hline Pop (1) & $\operatorname{Pop}(1)$ & 0 & Mov_pop(1) & Mov_pop(0) & 1 \\
\hline & & & Mov_mov(0) & Mov_mov(1) & 1 \\
\hline
\end{tabular}

Table 5. Saved original subsequence score

Table 6 shows the subtraction and addition of the original subsequence and new subsequence.

The final subsequence counts and the relative normal file counts are show in Table 5. The new score is also reflected as a difference in Table 7. Note that the "Add_pop" is a new counter.

\begin{tabular}{|l|l|l|l|}
\hline $\begin{array}{l}\text { Subtract original } \\
\text { subsequence }\end{array}$ & $\begin{array}{l}\text { Add new } \\
\text { subsequence }\end{array}$ & $\begin{array}{l}\text { Subtract original } \\
\text { opcode-pair count list }\end{array}$ & $\begin{array}{l}\text { Add new subsequence } \\
\text { opcode-pair count }\end{array}$ \\
\hline $\begin{array}{l}\text { Mov }(2-2=0) \\
\text { Add }(1-1=0)\end{array}$ & Mov $(0+2=2)$ & Mov_add $(1-1=0)$ & Mov_add $(0+1=1)$ \\
Pop $(1-1=0)$ & Add $(0+1=1)$ & Add_mov(1-1=0) & Add_mov $(0+0=0)$ \\
& Pop $(0+1=1)$ & Mov_pop $(1-1=0)$ & Mov_pop $(0+0=0)$ \\
& & Mov_mov(0) & $\begin{array}{l}\text { Add_mov(0+1=1) } \\
\text { Addpop }(1)\end{array}$ \\
\hline
\end{tabular}

Table 6. Subtract from old count and add new count

\begin{tabular}{|c|c|c|c|c|c|}
\hline $\begin{array}{l}\text { New Virus } \\
\text { opcode count } \\
\text { list }\end{array}$ & $\begin{array}{l}\text { Normal file } \\
\text { opcode count } \\
\text { list }\end{array}$ & $\begin{array}{l}\text { Difference } \\
\text { after changes }\end{array}$ & $\begin{array}{l}\text { new Virus opcode } \\
\text { sequence count } \\
\text { list }\end{array}$ & $\begin{array}{l}\text { Normal file } \\
\text { opcode sequence } \\
\text { count list }\end{array}$ & $\begin{array}{l}\text { Difference } \\
\text { after changes }\end{array}$ \\
\hline $\begin{array}{l}\text { Mov (2) } \\
\text { Add (1) } \\
\text { Pop(1) }\end{array}$ & $\begin{array}{l}\text { Mov (2) } \\
\text { Add (0) } \\
\text { Pop(1) }\end{array}$ & $\begin{array}{l}0 \\
1 \\
0\end{array}$ & $\begin{array}{l}\text { Mov_add (1) } \\
\text { Add_mov(0) } \\
\text { Mov_pop(0) } \\
\text { Mov_mov(1) } \\
\text { Add_pop(1) }\end{array}$ & $\begin{array}{l}\text { Mov_add (0) } \\
\text { Add_mov(0) } \\
\text { Mov_pop(0) } \\
\text { Mov_mov(1) } \\
\text { Add_pop(0) }\end{array}$ & $\begin{array}{l}1 \\
0 \\
0 \\
0 \\
1\end{array}$ \\
\hline
\end{tabular}

Table 7. New score after changes

As shown in Table 7, the new score of the affected subsequence is 3 , and the original score is 5 (shown in Table 5). This tells us that if we do such transposition, we will make the virus file closer to the normal file by 2 points.

\subsubsection{Updating the Changes}


This method is similar to the scoring-the-changes method except it will make permanent changes to the master lists.

For the transpose change, as shown in the previous section, the master score will be decreased from 8 to 6 (since we improved the score by 2). The master lists of the virus file will be updated, as highlighted in Table 8.

\begin{tabular}{|l|l|l|l|l|l|}
\hline $\begin{array}{l}\text { Virus opcode } \\
\text { count list }\end{array}$ & $\begin{array}{l}\text { Normal file } \\
\text { opcode count } \\
\text { list }\end{array}$ & difference & $\begin{array}{l}\text { Virus opcode-pair } \\
\text { count list }\end{array}$ & $\begin{array}{l}\text { Normal file } \\
\text { opcode-pair count } \\
\text { list }\end{array}$ & difference \\
\hline Mov (2) & Mov (2) & 0 & Mov_add (1) & Mov_add (0) & 1 \\
Add (1) & Add (0) & 1 & Add_mov(0) & Add_mov(0) & 0 \\
Pop (1) & Pop(1) & 0 & Mov_pop(0) & Mov_pop(0) & 0 \\
Retn(1) & Retn(1) & 0 & Pop_retn(1) & Pop_retn(1) & 0 \\
Sub (0) & Sub (1) & 1 & Mov_mov(1) & Mov_mov(1) & 0 \\
& & & Mov_sub(0) & Mov_sub(1) & 1 \\
& & Sub_pop(0) & Sub_pop(1) & 1 \\
\hline
\end{tabular}

\section{Table 8. Updated opcode count lists}

\subsubsection{Dead Code Insertion}

Instructions in our base virus are statistically different than normal programs. Previous work [5] has analyzed the statistics of virus instructions vs. normal program instructions. Their statistics are shown below in Figure 17 and Figure 18.

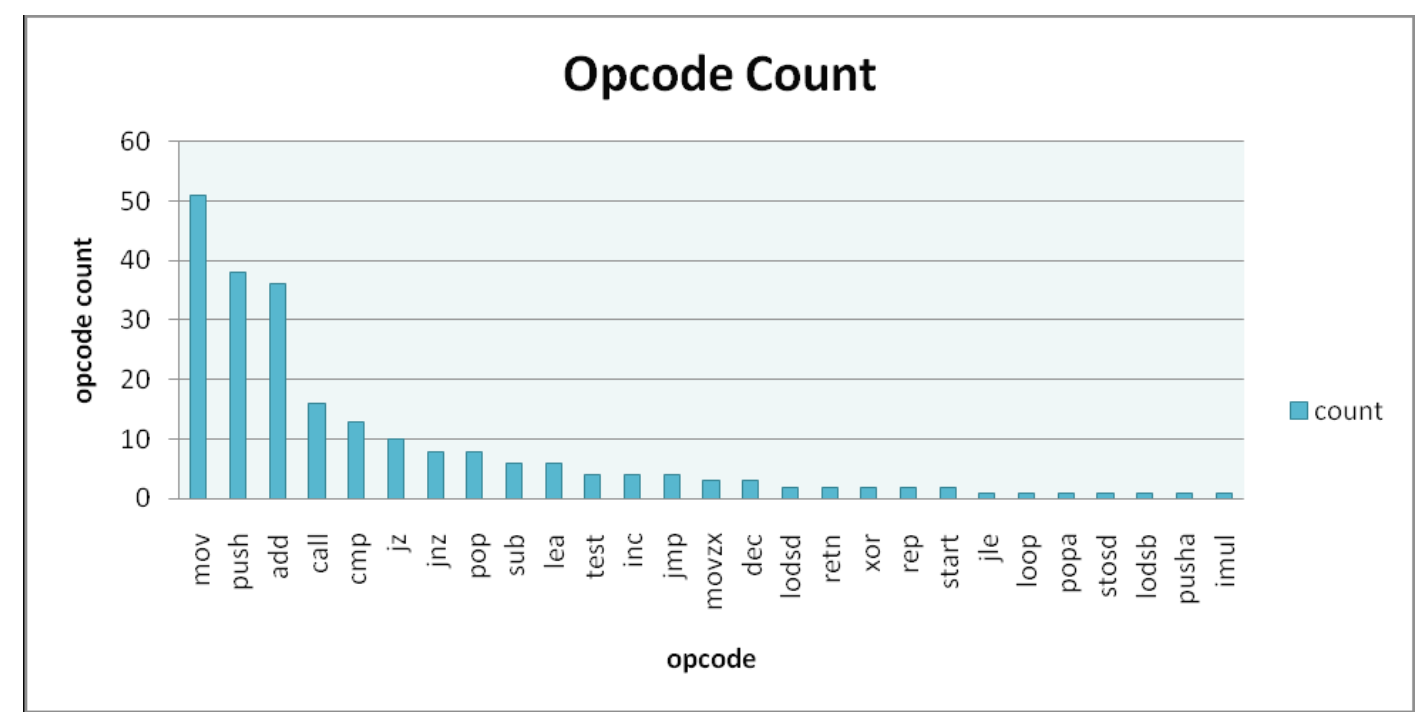

Figure 17. Base virus opcodes and their frequencies [5] 


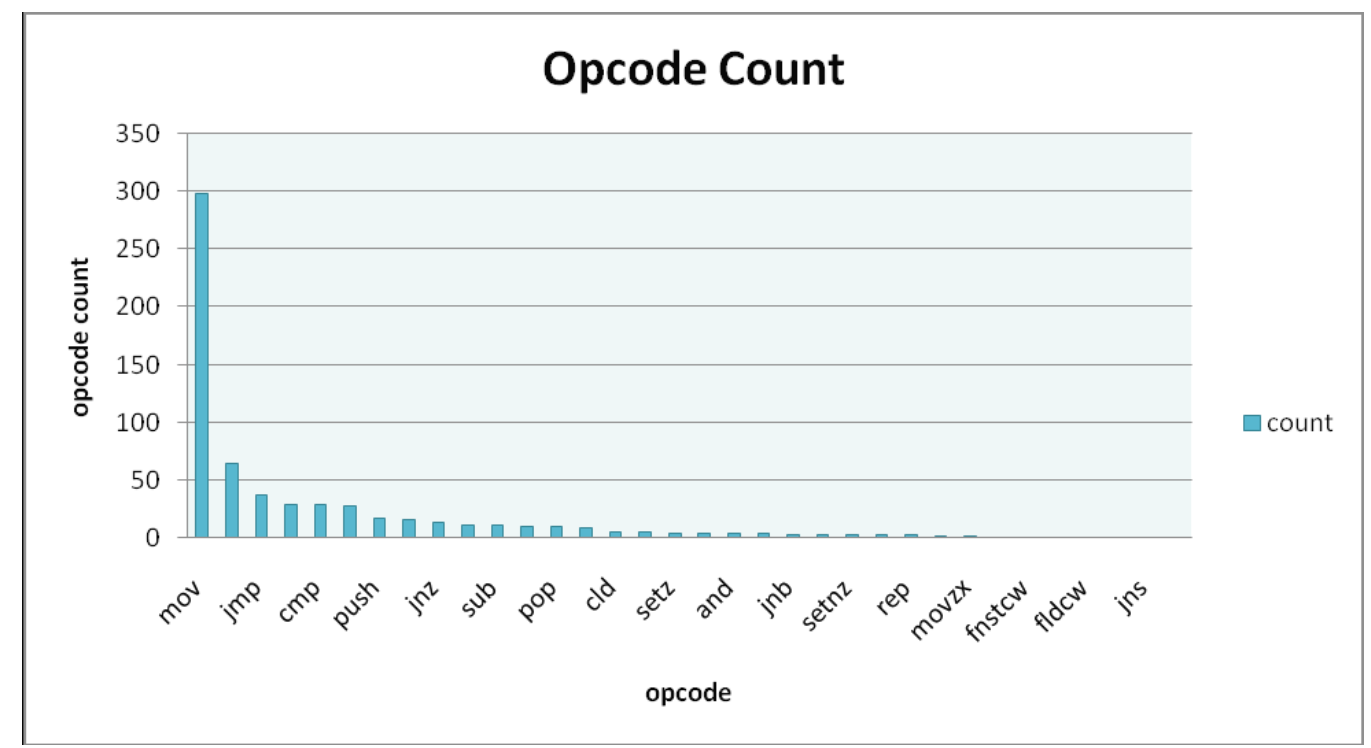

Figure 18. Opcodes of normal files and their frequencies [5]

In order to make our base virus more like a normal program, we will insert some dead code into the generated viruses. However, the set of dead codes that we built into our engine is finite. Applying these dead codes over and over will result in the HMM detector recognizing them. Therefore, we will also generate "dead codes" on the fly by copying blocks of instructions and subroutines from the normal program. Since the dynamically generated "dead codes" are "real codes" in the normal program, inserting them into our viruses will make our viruses look more like normal programs.

\subsubsection{Inserting Dead Code}

Our engine leveraged the build-in dead code library from the work done in [5]. However, instead of randomly inserting dead codes, we will try to insert some dead code before and/or after each instruction in a virus file only if that makes our virus more like a normal program. Our build-in dead code insertion algorithm is shown in Figure 19.

For each instruction

1. Insert dead code before and/or after it.

2. Compute a score using the Dynamic Scoring Algorithm.

3. If the score is better or remains the same, keep the changes and update the Dynamic Scoring Algorithm counters. 
4. If the score worsens, discard the change made in step 1.

\section{Figure 19. Dead code insertion algorithm}

\subsubsection{Inserting Dead Code Generated from a Normal File}

Since the build-in dead code library only included a limited set of instruction combinations, applying them into many virus copies will provide a pattern for HMM to detect the generated viruses. In order to overcome this limitation, we added logics into our engine to copy sets of instructions from normal files. Theoretically, this implementation will allow us to generate infinite sets of dead codes, since there is an infinite number of normal files.

The instruction sets that we copied from a normal file can function as a block of five or more continuous instructions, or as a complete subroutine. If we are copying a block of continuous instructions, we will insert an unconditional "jmp" instruction before the block so that these instructions will not be executed. In addition to inserting a "jmp" instruction, we might also need to modify the operands of some instructions so that the generated virus assembly file can be recompiled by FASM [21]. For example, if an instruction contains a label that is only valid in the normal file, then we will need to replace that label with a label that is valid in the generated virus file.

When copying a subroutine, we also need to modify the operands of some instructions. However, we do not need to insert jump instructions. The copied subroutine will be placed between two subroutines in the virus file. Since the copied subroutine never gets called, it will not impact the original behaviors of the virus.

\subsubsection{Equivalent Instruction Substitution}

Some opcodes, such as mov, push, and add instructions, appear more frequently in the base virus [5]. To make the generated virus' opcode count statistically closer to normal programs, we 
substitute equivalent instructions in place of these instructions. For example, "add instruction" can be replaced with "sub," "lea," or "not," followed by "neg" instructions, as shown in Table 9.

\begin{tabular}{|c|c|}
\hline add $\mathrm{R}$, imm & $\begin{array}{l}\text { 1. sub } R, \text { new_imm where new_imm }=\text { imm } \times(-1) \\
\text { 2. lea } R,[R+\text { imm] }\end{array}$ \\
\hline add $R, 1$ & $\begin{array}{l}\text { 1. not R } \\
\text { neg R }\end{array}$ \\
\hline
\end{tabular}

Table 9. Equivalent instructions for add [5]

While substituting equivalent instructions, we also keep track of the score changes between virus copies and normal files. We only perform substitution if the score improves. Our algorithm for equivalent instruction substitution is shown in Figure 20 below:

For each instruction

1. Perform substitution if possible.

2. Compute a score using the Dynamic Scoring Algorithm.

3. If the score improves or remains the same, keep the change and update the Dynamic Scoring Algorithm counters.

4. If the score worsens, discard the change made in step 1.

\section{Figure 20. Equivalent instruction substitution algorithm}

\subsubsection{Transposition}

After generating dead code and performing equivalent instruction substitution, we perform transposition to make our virus even closer to a normal program. The transposition algorithm in [5] performs transposition randomly with a probability of $25 \%$. We removed the randomized nature of that algorithm. Instead, we used the Dynamic Scoring Algorithm to perform transposition in order to make our virus closer to a normal program. Therefore, our final transposition algorithm is as shown in Figure 21:

1. Read two instructions with two operands.

2. To perform transposition: 
a. Read third instruction

b. If the third instruction is not any conditional jump instruction then

i. If the to-operands of both instructions are not equal

And

If the to-operand of the first instruction is not equal to the from-operand of the second instruction

and

If the from-operand of the first instruction is not equal to the to-operand of the second instruction

then

Swap the two instructions.

3. Compute the score of the transposition.

4. If the score improves, keep the change. Otherwise, discard the change.

\section{Figure 21. Transposition algorithm}

\subsection{Metamorphic Engine Algorithm}

The code obfuscation techniques described in section 4.3 are implemented as individual modules in our engine. We execute each module in sequence to generate our final virus copy. The overall engine algorithm is shown in Figure 22 below:

1. Read in a base virus and a normal file.

2. Initialize the Dynamic Scoring Algorithm.

3. Insert dead code between each instruction if it makes the virus "closer" to the normal file.

4. Perform equivalent instruction substitution for each instruction if it makes the virus closer to the normal file.

5. Perform transposition for every instruction pair if it makes the virus closer to a normal file.

6. For each instruction, generate a random number between 0 and 99:

a. If random number < configured percentage for junk block, copy a junk block from normal file. 
7. Between each subroutine, generate a random number between 0 and 99.

a. If random number < configured percentage for junk function, copy a subroutine from normal file.

\section{Figure 22. Metamorphic engine algorithm}

\section{Experiments}

We used the similarity test and HMM test tools developed in [2] to perform our test. After we successfully demonstrated that our engine was able to escape HMM-based detection, we repeated our test with different engine settings (i.e., reduced the number of dead code copied from the normal file to find the threshold at which the HMM detector began to fail.

\subsection{Base Virus}

To test our engine, we first used NGVCK to generate 200 virus files of the same family. These 200 virus files served as our base viruses. We then constructed 40 normal files from cygwin utility files.

After we generated our base viruses and normal files, we used the HMM detector to verify that viruses generated by NGVCK were still detectable. The procedures we executed with the HMM detector are the same as those in [2]. We first generated the HMM model with 160 viruses. We then generated the scores for the remaining 40 viruses against the HMM model. We also generated the scores for the 40 normal files against the same HMM model.

If none of the normal files score higher than the viruses, then the HMM detector will be able to detect the family viruses, since there is a threshold that it could use to determine whether a given file is a virus (score higher than the threshold) or a normal file (score lower than the threshold). On the other hand, if some normal files score higher than some of the virus files, then the HMM detector will not have a good threshold to determine a given file. This means that some viruses will escape HMM-based detection. 
Figure 23 shows the result of base viruses against normal files. All of the normal files score lower than virus files. Therefore, the base viruses we generated from NGVCK are detectable by the HMM detector.

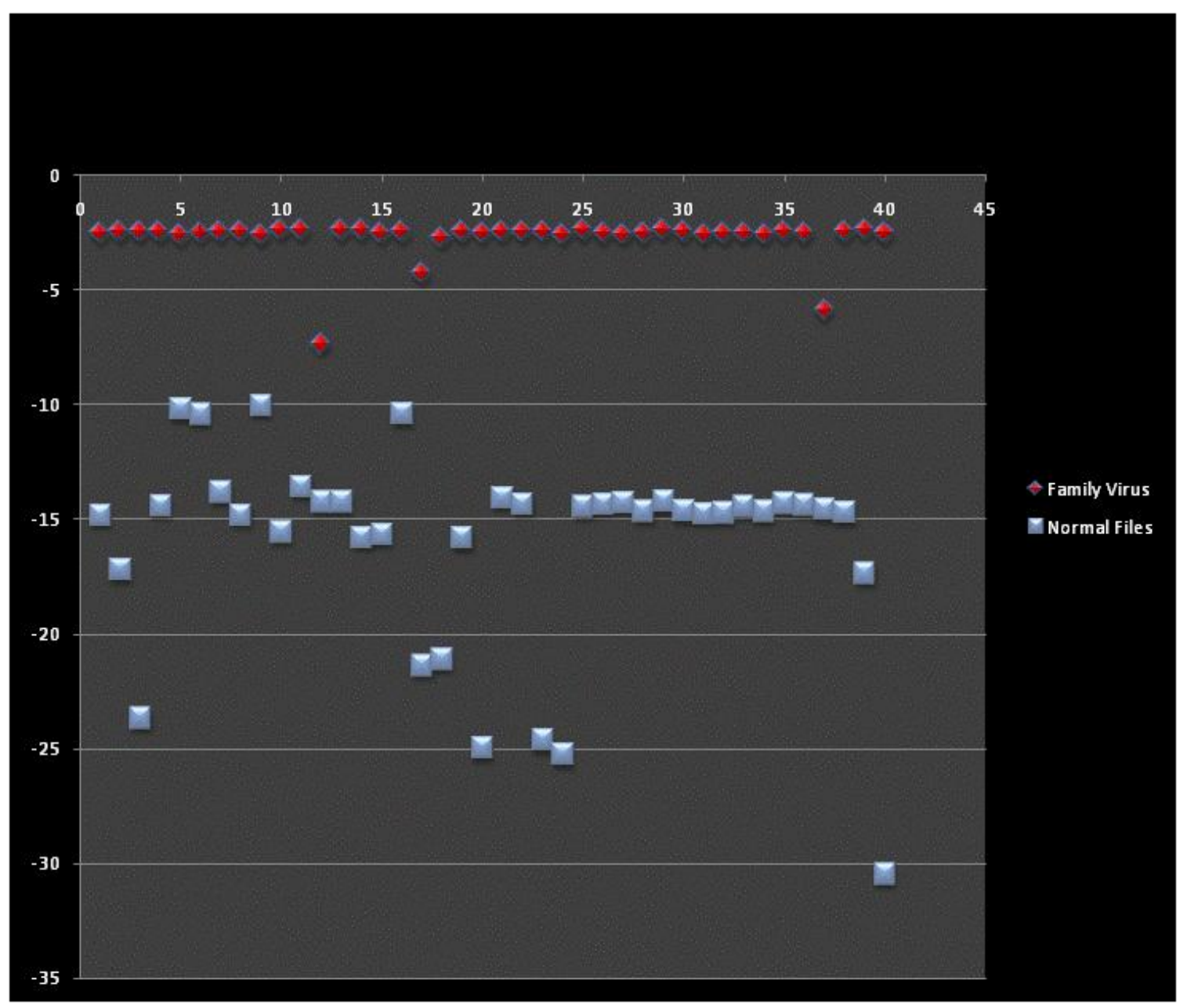

Figure 23. HMM results for base viruses generated by NGVCK

After we generated base viruses, we used our engine to perform additional code obfuscation. Our engine will take one base virus and one normal file as inputs. It will apply additional code obfuscation techniques to the base virus so that the generated virus copy will be statistically closer to a normal file. In our experiment, we will make five virus copies closer to one normal file. For example, virus copies 1, 41, 81, 121, and 161 will look like normal file 1. Virus copies 2, 42, 82, 122, and 162 will look like normal file 2. 


\subsection{Similarity Test}

The similarity test compares and reports the percentage of similarity among two assembly programs [5]. Since our engine will make a virus file closer to a normal file, we will compare the similarity of a virus file with its peer normal file as we increase the percent of dead code copied from the normal file.

We first compared a base virus against a normal file, and there was no similarity between them at all. Then we ran the two files through our engine without any dead code copying configured. We were able to obtain a similarity score of $13.8 \%$ between the two files. After that, we copied dead code from the normal file into the virus and computed the similarity score again. The more dead code we copied from the normal file into the virus, the higher the similarity score, which is what we expected. Table 10 shows the table format of the similarity scores as we increased the file sizes by copying more dead codes.

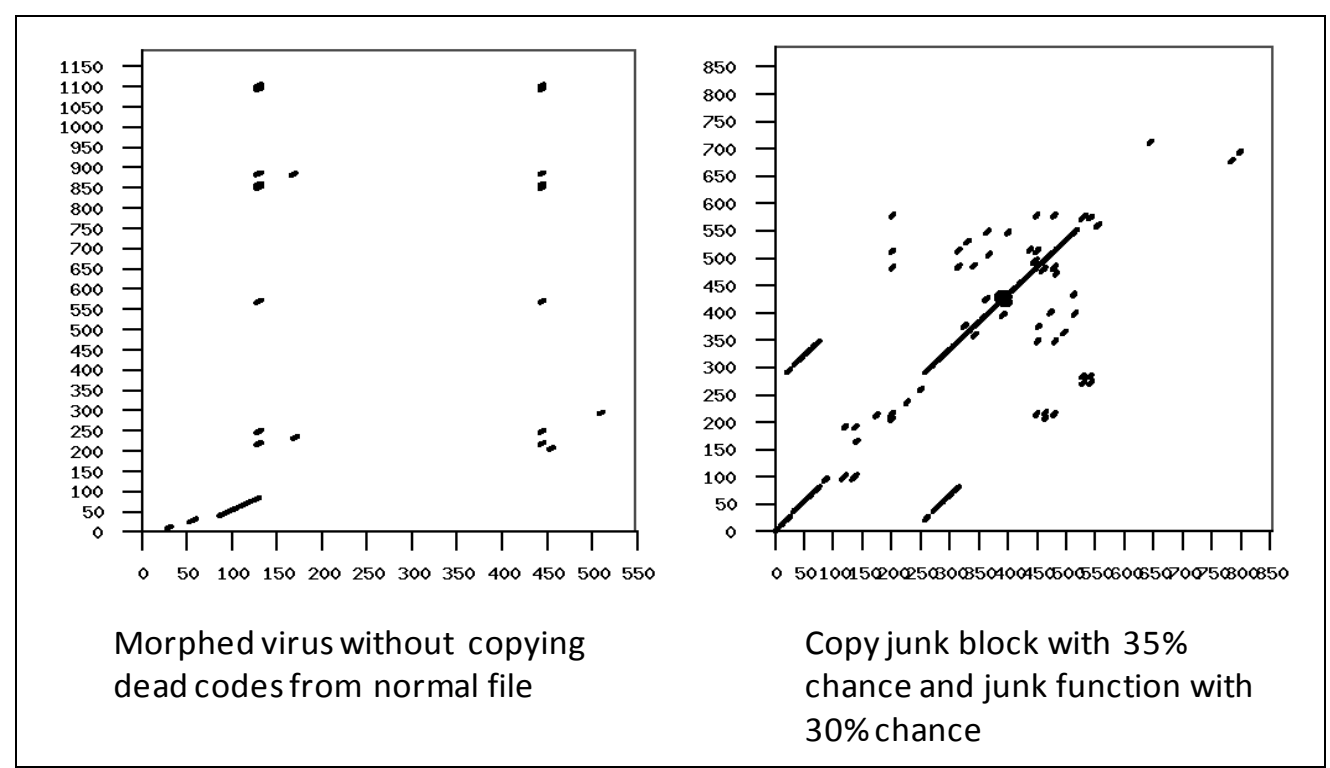

Figure 24. Similarity score for morphed virus against normal files

\begin{tabular}{|l|c|c|}
\hline & File size & Similarity Score \\
\hline Base virus & $17 \mathrm{~K}$ & 0 \\
\hline $\begin{array}{l}\text { Morphed virus without copying dead codes } \\
\text { from normal file }\end{array}$ & $21.8 \mathrm{~K}$ & $13.8 \%$ \\
\hline
\end{tabular}




\begin{tabular}{|l|c|c|}
\hline Copy junk block with 10\% chance & $23.2 \mathrm{~K}$ & $15.8 \%$ \\
\hline Copy junk block with 25\% chance & $23.8 \mathrm{~K}$ & $16.2 \%$ \\
\hline Copy junk block with 35\% chance & $24.3 \mathrm{~K}$ & $15.0 \%$ \\
\hline $\begin{array}{l}\text { Copy junk block with 35\% chance and junk } \\
\text { function with 5\% chance }\end{array}$ & $25.8 \mathrm{~K}$ & $16.5 \%$ \\
\hline $\begin{array}{l}\text { Copy junk block with 35\% chance and junk } \\
\text { function with 15\% chance }\end{array}$ & $26.3 \mathrm{~K}$ & $16.6 \%$ \\
\hline $\begin{array}{l}\text { Copy junk block with 35\% chance and junk } \\
\text { function with 20\% chance }\end{array}$ & $28.5 \mathrm{~K}$ & $17.2 \%$ \\
\hline $\begin{array}{l}\text { Copy junk block with 35\% chance and junk } \\
\text { function with 30\% chance }\end{array}$ & $28.6 \mathrm{~K}$ & $18.1 \%$ \\
\hline
\end{tabular}

\section{Table 10. Similarity score of virus and its peer normal file}

Since dead code blocks copied from the normal file were of different sizes, we will use the file size for the $\mathrm{X}$-axis of our graphs to show our experiment results for the rest of this report. Figure 25 shows the similarity scores for the generated viruses against normal files. As more dead code was copied from the normal file, the virus file size increased. The similarity score between the virus and normal file also increased as expected.

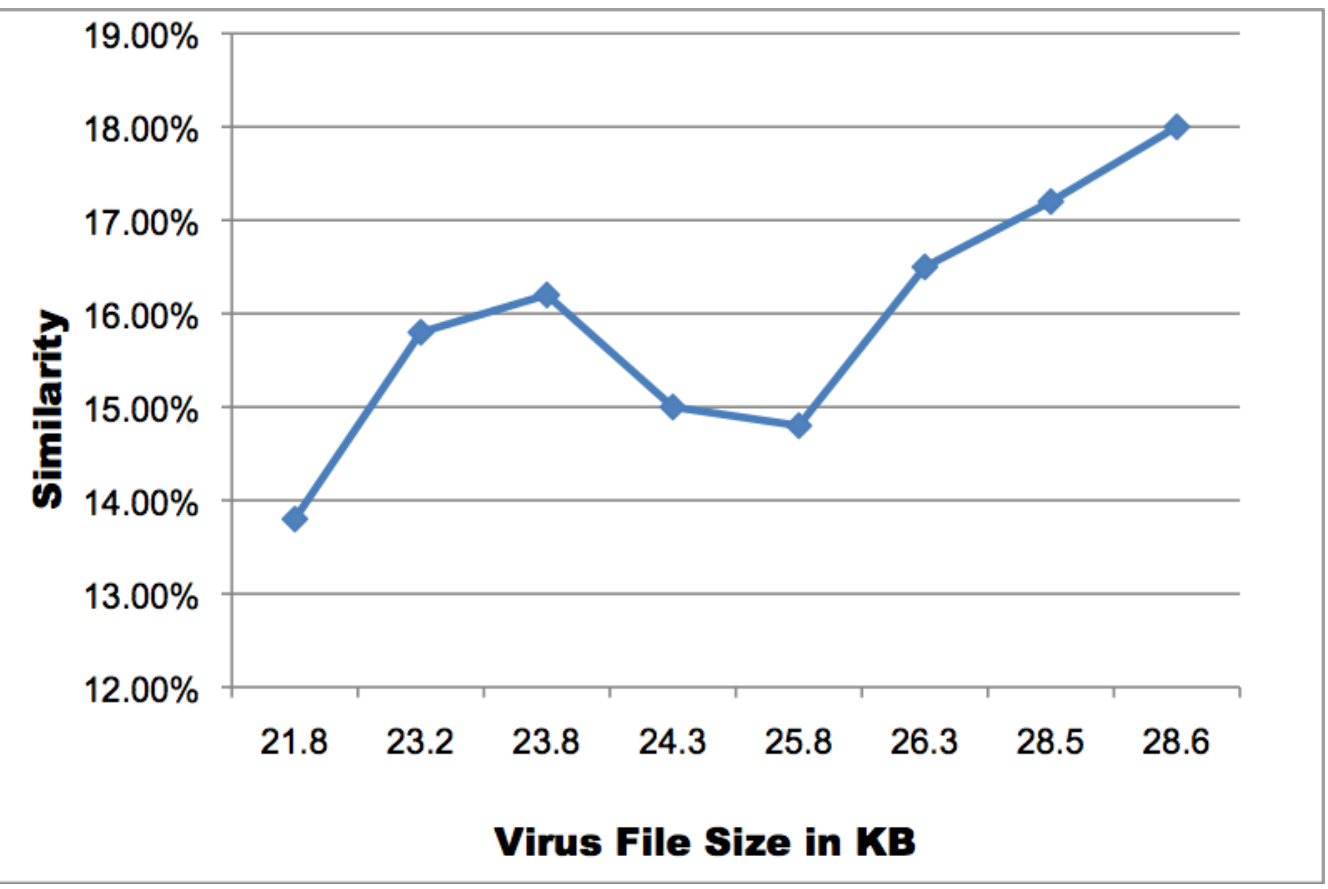

Figure 25. Similarity score of virus and normal file 


\section{$5.3 \mathrm{HMM}$}

As expected, similarity tests show that copying more dead code from a normal file into a virus makes the virus closer to the normal file. We will perform HMM tests for each set of viruses that we generate. The idea is to see how much dead code we need to copy from normal files in order to make our viruses undetectable.

We executed HMM tests on our viruses with a number of hidden states from 2 to 5 . However, based on the previous work $[2,5]$ and our results, it appears that the number of hidden states will not affect the results. Therefore, we will focus on analyzing the results for the HMM tests with three hidden states. We will include some results with different numbers of hidden states in appendix A.

For all our tests, we constructed the HMM model from 160 virus files. Then, we compared the remaining 40 virus files against the HMM model. We also compared the 40 normal files against the HMM model.

\subsubsection{Zero Percent Dead Code}

This set of viruses was generated by applying our engine without any dead code copying from normal files. With this setting, the average file size increase from $17 \mathrm{~KB}$ to $21.8 \mathrm{~KB}$. The similarity score also increase from $0 \%$ to $13.8 \%$.

\begin{tabular}{|ll|ll|ll|ll|}
\hline \multicolumn{7}{|c|}{ 0\% dead code with N=3 } \\
\hline \multicolumn{7}{|c|}{ Family Viruses } & \multicolumn{4}{c|}{ Normal Files } \\
\hline N120 & -3.82742 & N140 & -2.64343 & IDAR0 & -8.62106 & IDAR20 & -12.8004 \\
N121 & -2.60846 & N141 & -2.78598 & IDAR1 & -5.98894 & IDAR21 & -8.56907 \\
N122 & -2.74388 & N142 & -3.07189 & IDAR2 & -13.8067 & IDAR22 & -15.1685 \\
N123 & -2.93378 & N143 & -2.74519 & IDAR3 & -18.3758 & IDAR23 & -16.5805 \\
N124 & -2.74935 & N144 & -2.70781 & IDAR4 & -4.98846 & IDAR24 & -8.55813 \\
N125 & -2.83254 & N145 & -2.71075 & IDAR5 & -9.09981 & IDAR25 & -8.41451 \\
N126 & -2.649 & N146 & -2.6337 & IDAR6 & -12.527 & IDAR26 & -8.22832 \\
N127 & -2.76175 & N147 & -2.77156 & IDAR7 & -12.4914 & IDAR27 & -8.6588 \\
N128 & -2.7331 & N148 & -2.68296 & IDAR8 & -8.16147 & IDAR28 & -8.21336 \\
N129 & -3.01979 & N149 & -3.92464 & IDAR9 & -9.20784 & IDAR29 & -8.49555 \\
N130 & -4.03604 & N150 & -2.7027 & IDAR10 & -10.5268 & IDAR30 & -10.7358 \\
N131 & -2.57982 & N151 & -2.75938 & IDAR11 & -6.27038 & IDAR31 & -8.62145 \\
\hline
\end{tabular}




\begin{tabular}{|ll|ll|ll|ll|}
\hline N132 & -2.75932 & $\mathrm{~N} 152$ & -2.64362 & IDAR12 & -6.27038 & IDAR32 & -8.18047 \\
N133 & -2.75863 & N153 & -4.08823 & IDAR13 & -14.3691 & IDAR33 & -9.47778 \\
N134 & -2.57266 & N154 & -2.49181 & IDAR14 & -11.6093 & IDAR34 & -8.41929 \\
N135 & -2.73927 & N155 & -2.81595 & IDAR15 & -8.44406 & IDAR35 & -8.75353 \\
N136 & -2.63524 & N156 & -2.79971 & IDAR16 & -16.2526 & IDAR36 & -8.53441 \\
N137 & -2.72739 & N157 & -2.73247 & IDAR17 & -11.8591 & IDAR37 & -11.3747 \\
N138 & -3.91962 & N158 & -2.61171 & IDAR18 & -12.5659 & IDAR38 & -11.1086 \\
N139 & -2.91821 & N159 & -2.62985 & IDAR19 & -15.9829 & IDAR39 & -23.4772 \\
\hline \multicolumn{3}{|c|}{ Min Score $=-4.088$} & \multicolumn{3}{c|}{ Max Score $=-4.988$} \\
\hline \multicolumn{3}{|c|}{ 0 viruses with scores <-4.988 files with scores > -4.088 }
\end{tabular}

Table 11. HMM Results with $0 \%$ dead code copied

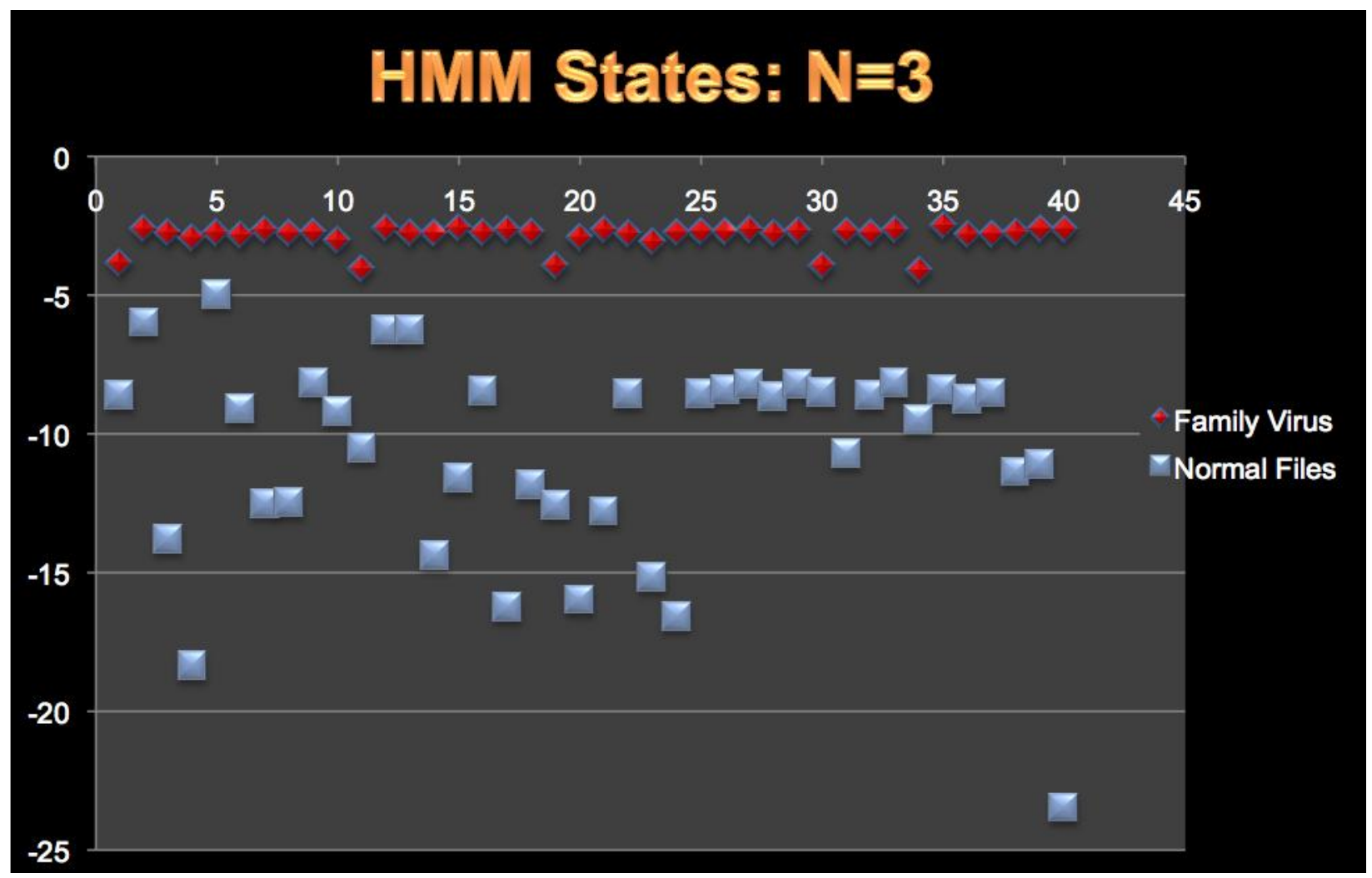

Figure 26. HMM result with $0 \%$ dead code copied

\subsubsection{Copying Blocks of Dead Code from Normal File}

This set of viruses was generated by applying our engine with a probability of $10 \%$ to $35 \%$ for copying dead code blocks into viruses for each virus instruction. With this setting, the average file size increased from $17 \mathrm{~KB}$ to $24.3 \mathrm{~KB}$. 
Even with the higher percentage setting at 35\%, the HMM was still able to recognize the family virus. However, we observed that the maximum score of normal files increased as we increased the percentage. Figure 27 shows the maximum score of the normal files as we increased the percentage of dead code copied. The HMM results for $35 \%$ dead code copied are shown in Figure 28 and Table 12.

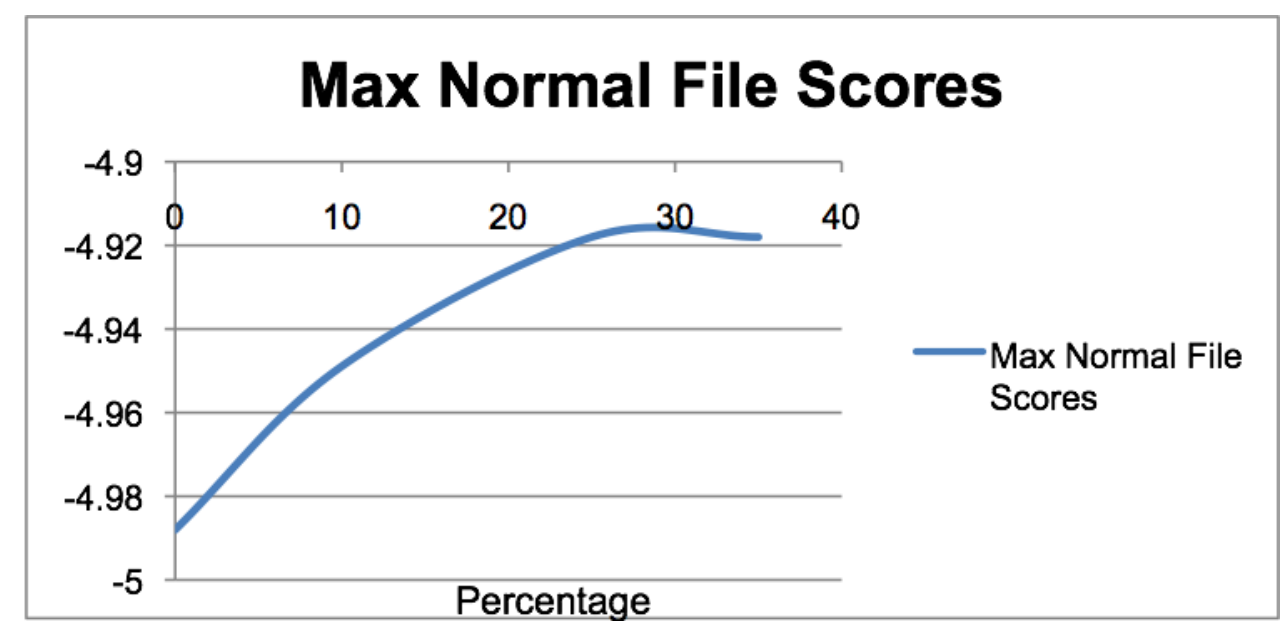

Figure 27. Maximun normal file scores vs. percentage increase

\begin{tabular}{|ll|ll|ll|ll|}
\hline \multicolumn{7}{|c|}{ 35\% dead code copying model with N=3 } \\
\hline \multicolumn{7}{|c|}{ Family Viruses } & \multicolumn{4}{c|}{ Normal Files } \\
\hline IDAN120 & -2.540 & IDAN140 & -2.044 & IDAR0 & -8.529 & IDAR20 & -12.926 \\
IDAN121 & -1.930 & IDAN141 & -2.102 & IDAR1 & -5.906 & IDAR21 & -8.473 \\
IDAN122 & -2.051 & IDAN142 & -2.405 & IDAR2 & -13.798 & IDAR22 & -14.865 \\
IDAN123 & -2.439 & IDAN143 & -2.386 & IDAR3 & -18.345 & IDAR23 & -16.304 \\
IDAN124 & -2.138 & IDAN144 & -2.387 & IDAR4 & -4.889 & IDAR24 & -8.460 \\
IDAN125 & -2.171 & IDAN145 & -2.397 & IDAR5 & -9.212 & IDAR25 & -8.316 \\
IDAN126 & -2.129 & IDAN146 & -2.392 & IDAR6 & -12.640 & IDAR26 & -8.131 \\
IDAN127 & -2.383 & IDAN147 & -2.384 & IDAR7 & -12.589 & IDAR27 & -8.563 \\
IDAN128 & -2.444 & IDAN148 & -2.372 & IDAR8 & -8.299 & IDAR28 & -8.117 \\
IDAN129 & -2.309 & IDAN149 & -2.938 & IDAR9 & -9.151 & IDAR29 & -8.400 \\
IDAN130 & -2.707 & IDAN150 & -2.343 & IDAR10 & -10.643 & IDAR30 & -10.617 \\
IDAN131 & -2.091 & IDAN151 & -2.467 & IDAR11 & -6.190 & IDAR31 & -8.527 \\
IDAN132 & -2.497 & IDAN152 & -2.241 & IDAR12 & -6.190 & IDAR32 & -8.084 \\
\hline
\end{tabular}




\begin{tabular}{|cl|ll|ll|lr|}
\hline IDAN133 & -2.479 & IDAN153 & -2.970 & IDAR13 & -14.500 & IDAR33 & -9.386 \\
IDAN134 & -2.032 & IDAN154 & -2.155 & IDAR14 & -11.527 & IDAR34 & -8.328 \\
IDAN135 & -2.268 & IDAN155 & -2.295 & IDAR15 & -8.563 & IDAR35 & -8.663 \\
IDAN136 & -2.079 & IDAN156 & -2.472 & IDAR16 & -16.196 & IDAR36 & -8.440 \\
IDAN137 & -2.085 & IDAN157 & -2.411 & IDAR17 & -11.622 & IDAR37 & -11.259 \\
IDAN138 & -3.301 & IDAN158 & -2.418 & IDAR18 & -12.509 & IDAR38 & -11.030 \\
IDAN139 & -2.331 & IDAN159 & -2.082 & IDAR19 & -15.686 & IDAR39 & -23.439 \\
\hline \multicolumn{3}{|c|}{ Min Score $=-3.301$} & \multicolumn{3}{c|}{ Max Score $=-4.889$} \\
\hline \multicolumn{3}{|c|}{ viruses with scores $<-4.889$} & niles with scores $>-3.301$ \\
\hline
\end{tabular}

Table 12. HMM results with $35 \%$ dead code copied

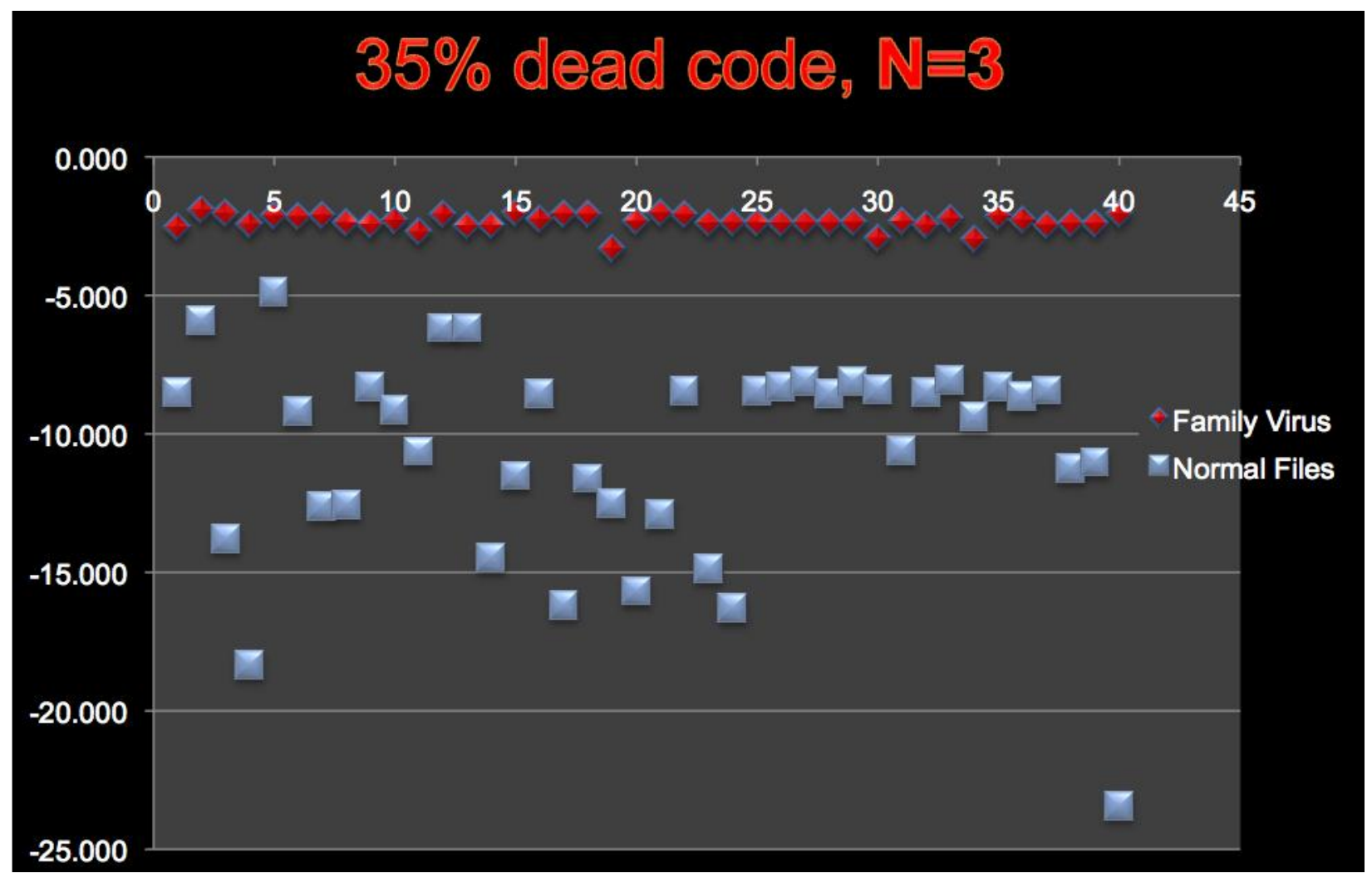

Figure 28. HMM results with $35 \%$ dead code copied

\subsubsection{Copying Subroutines and Blocks of Dead Code from Normal File}

We continued our experiment by adding subroutines copied from the normal files into our viruses. We first configured the sub-routine copying probability to $5 \%$ and ran our test. We repeated our tests with different sub-routine copying probabilities of $15 \%, 20 \%$, and $30 \%$. 
Even with a small probability of $5 \%$, we started to see some viruses score lower than the maximum score of the normal files.

Table 13 and Figure 29 show the scores of family viruses and normal files against the HMM model. Sixteen viruses score lower than the maximum normal file score. This means that if the HMM threshold is set to the maximum normal file score, 16 viruses will be undetectable by the HMM. On the other hand, if the HMM threshold is set to the minimum virus file score, then three normal files will be classified as viruses.

\begin{tabular}{|c|c|c|c|c|c|c|c|}
\hline \multicolumn{8}{|c|}{$35 \%$ dead code block, and $5 \%$ subroutine copying model with $\mathrm{N}=3$} \\
\hline \multicolumn{4}{|c|}{ Family Viruses } & \multicolumn{4}{|c|}{ Normal Files } \\
\hline IDAN120 & -2.441 & IDAN140 & -2.069 & IDARO & -3.700 & IDAR20 & -3.193 \\
\hline IDAN121 & -1.960 & IDAN141 & -2.111 & IDAR1 & -3.506 & IDAR21 & -3.040 \\
\hline IDAN122 & -2.014 & IDAN142 & -2.432 & IDAR2 & -4.533 & IDAR22 & -7.914 \\
\hline IDAN123 & -2.482 & IDAN143 & -2.431 & IDAR3 & -3.541 & IDAR23 & -7.806 \\
\hline IDAN124 & -2.170 & IDAN144 & -2.388 & IDAR4 & -3.286 & IDAR24 & -3.021 \\
\hline IDAN125 & -2.140 & IDAN145 & -2.421 & IDAR5 & -3.281 & IDAR25 & -3.013 \\
\hline IDAN126 & -2.154 & IDAN146 & -2.379 & IDAR6 & -3.292 & IDAR26 & -3.008 \\
\hline IDAN127 & -2.431 & IDAN147 & -2.413 & IDAR7 & -4.321 & IDAR27 & -3.019 \\
\hline IDAN128 & -2.474 & IDAN148 & -2.368 & IDAR8 & -3.268 & IDAR28 & -3.009 \\
\hline IDAN129 & -2.275 & IDAN149 & -2.940 & IDAR9 & -3.103 & IDAR29 & -3.017 \\
\hline IDAN130 & -2.720 & IDAN150 & -2.395 & IDAR10 & -3.731 & IDAR30 & -2.428 \\
\hline IDAN131 & -2.115 & IDAN151 & -2.554 & IDAR11 & -3.212 & IDAR31 & -3.018 \\
\hline IDAN132 & -2.492 & IDAN152 & -2.162 & IDAR12 & -3.212 & IDAR32 & -3.007 \\
\hline IDAN133 & -2.515 & IDAN153 & -2.980 & IDAR13 & -4.459 & IDAR33 & -3.029 \\
\hline IDAN134 & -2.079 & IDAN154 & -2.233 & IDAR14 & -2.882 & IDAR34 & -3.016 \\
\hline IDAN135 & -2.311 & IDAN155 & -2.322 & IDAR15 & -3.187 & IDAR35 & -3.033 \\
\hline IDAN136 & -2.191 & IDAN156 & -2.527 & IDAR16 & -4.355 & IDAR36 & -3.006 \\
\hline IDAN137 & -2.123 & IDAN157 & -2.456 & IDAR17 & -4.114 & IDAR37 & -2.424 \\
\hline IDAN138 & -2.643 & IDAN158 & -2.406 & IDAR18 & -3.057 & IDAR38 & -3.658 \\
\hline IDAN139 & -2.514 & IDAN159 & -2.122 & IDAR19 & -7.818 & IDAR39 & -8.249 \\
\hline \multicolumn{4}{|c|}{ Min Score $=-2.980$} & \multicolumn{4}{|c|}{ Max Score $=-2.242$} \\
\hline \multicolumn{4}{|c|}{16 viruses with scores $<-2.242$} & \multicolumn{4}{|c|}{3 normal files with scores $>-2.980$} \\
\hline
\end{tabular}

Table 13. HMM results with $35 \%$ dead code blocks and $5 \%$ subroutine copied 


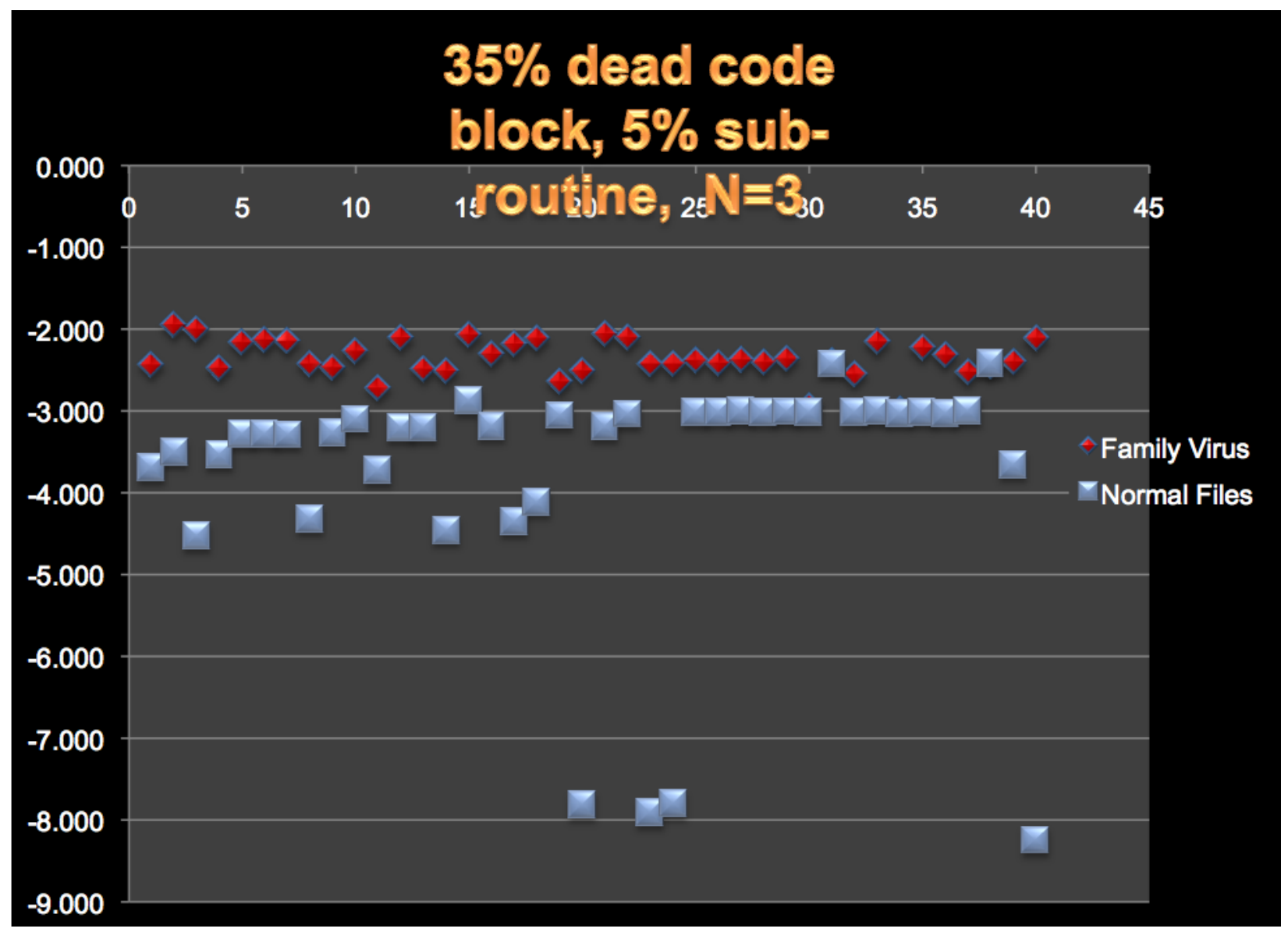

Figure 29. HMM result with $35 \%$ dead code blocks and $5 \%$ subroutine copied

\subsubsection{Copying Subroutines Only from Normal File}

Based on the results shown in the previous section, we noticed copying subroutines from normal files significantly impacted our scores. Therefore, we conducted additional experiments by copying only subroutines into our base viruses without additional code obfuscation. The results showed that even with as little as $5 \%$ subroutines copied from the normal files, we were able to make the HMM detector misclassify some of our viruses.

\begin{tabular}{|ll|ll|ll|ll|}
\hline \multicolumn{7}{|c|}{ 5\% subroutine copying model with N=3 } \\
\hline \multicolumn{3}{|c|}{ Family Viruses } & \multicolumn{4}{c|}{ Normal Files } \\
\hline IDAN120 & -3.465 & IDAN140 & -2.644 & IDAR0 & -5.886 & IDAR20 & -4.098 \\
IDAN121 & -2.731 & IDAN141 & -2.785 & IDAR1 & -4.786 & IDAR21 & -5.566 \\
IDAN122 & -2.671 & IDAN142 & -2.720 & IDAR2 & -8.619 & IDAR22 & -12.088 \\
IDAN123 & -2.708 & IDAN143 & -2.640 & IDAR3 & -4.688 & IDAR23 & -11.677 \\
IDAN124 & -2.595 & IDAN144 & -2.748 & IDAR4 & -4.428 & IDAR24 & -5.344 \\
IDAN125 & -2.623 & IDAN145 & -2.628 & IDAR5 & -4.530 & IDAR25 & -5.349 \\
IDAN126 & -2.585 & IDAN146 & -2.557 & IDAR6 & -4.509 & IDAR26 & -5.328 \\
\hline
\end{tabular}




\begin{tabular}{|c|c|c|c|c|c|c|c|}
\hline IDAN127 & -3.659 & IDAN147 & -2.634 & IDAR7 & -5.763 & IDAR27 & -5.324 \\
\hline IDAN128 & -2.664 & IDAN148 & -2.472 & IDAR8 & -4.524 & IDAR28 & -5.323 \\
\hline IDAN129 & -2.634 & IDAN149 & -4.361 & IDAR9 & -6.294 & IDAR29 & -5.472 \\
\hline IDAN130 & -4.252 & IDAN150 & -2.665 & IDAR10 & -5.150 & IDAR30 & -5.253 \\
\hline IDAN131 & -2.550 & IDAN151 & -2.700 & IDAR11 & -6.259 & IDAR31 & -5.309 \\
\hline IDAN132 & -2.521 & IDAN152 & -2.586 & IDAR12 & -6.259 & IDAR32 & -5.306 \\
\hline IDAN133 & -2.718 & IDAN153 & -3.779 & IDAR13 & -5.937 & IDAR33 & -5.275 \\
\hline IDAN134 & -2.670 & IDAN154 & -2.599 & IDAR14 & -3.276 & IDAR34 & -5.426 \\
\hline IDAN135 & -2.701 & IDAN155 & -2.720 & IDAR15 & -4.149 & IDAR35 & -5.295 \\
\hline IDAN136 & -2.714 & IDAN156 & -4.330 & IDAR16 & -10.162 & IDAR36 & -5.266 \\
\hline IDAN137 & -2.662 & IDAN157 & -2.550 & IDAR17 & -5.931 & IDAR37 & -5.318 \\
\hline IDAN138 & -2.441 & IDAN158 & -2.531 & IDAR18 & -5.649 & IDAR38 & -6.460 \\
\hline IDAN139 & -2.683 & IDAN159 & -2.543 & IDAR19 & -11.896 & IDAR39 & -12.879 \\
\hline \multicolumn{4}{|c|}{ Min Score $=-4.361$} & \multicolumn{4}{|c|}{ Max Score $=-3.276$} \\
\hline \multicolumn{4}{|c|}{6 viruses with scores $<-3.276$} & \multicolumn{4}{|c|}{3 normal files with scores $>-4.361$} \\
\hline
\end{tabular}

Table 14. HMM results with $5 \%$ subroutine copied

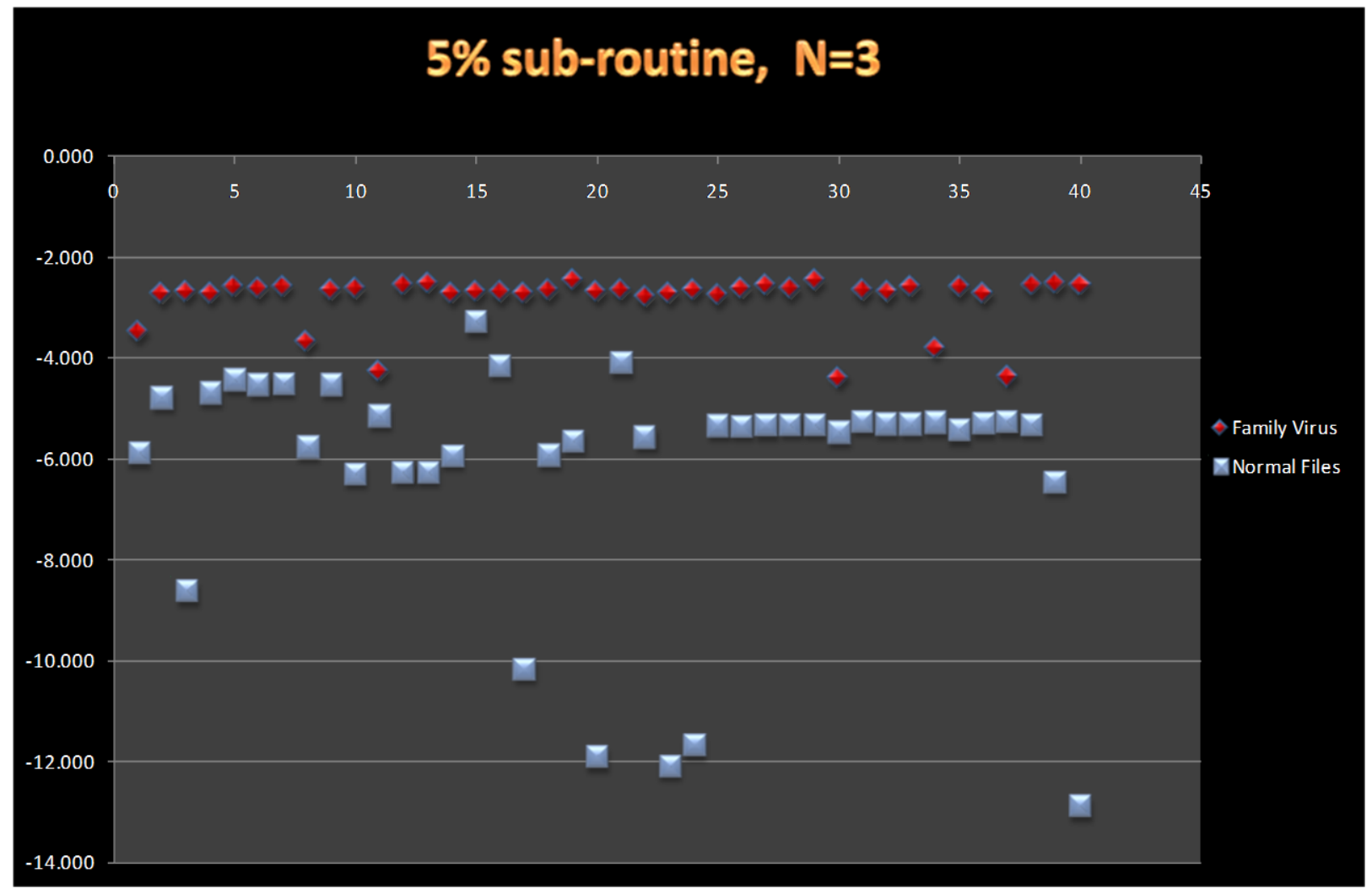

Figure 30. HMM results with 5\% subroutine copied

Additional experiment results can be found in Appendix A. 


\section{Detection Technique for Our Engine}

The engine developed in this project copies a fair amount of dead codes and subroutines from normal files. Dead codes are usually surrounded by a "jmp label_name" instruction and a label_name. Subroutines copied were not executed in the viral copies..

To detect viruses generated by this engine, we can develop a utility to remove these copied dead codes prior to using the HMM detection tool. The utility logic should be as follows:

1. Scan the file and record a block for each "jmp" and label pair. In addition, write down the names of all subroutines.

2. For each block, scan the file to see if other instructions might branch into it. If no instruction branches into it, remove it.

3. For each subroutine, scan the file to see if this subroutine is called by outside instructions. If not, remove it.

After a virus is pre-processed by the utility, it will be detectable by the HMM.

We experimented with our detection technique by developing a simple java program to remove the dead code copied from normal files. We first generated the morphed viruses by copying $30 \%$ subroutines from normal files. Then we used our program to remove the subroutines copied from normal files. We were able to obtain the same results, as we never copied any subroutines. Note that we trained our HMM model from files with subroutines removed. In other words, we reverted portions of what our engine did in order to detect viruses generated by our engine.

Since our detection method only scans unexecuted codes, virus writers can easily enhance the engine by including code to execute these dead codes with very small probability. For example, virus writers can embed code to first generate two large random numbers, then call the subroutine copied if the two random numbers are equal. The probability of two large consecutive generated random numbers being equal is almost 0 , but it will make the copied subroutines harder to remove, since these subroutines will now appear as they "might" be used. 


\section{Conclusions}

By making our viruses closer to normal files, we were able to make them undetectable using an HMM-based detector. The HMM-based detector began to fail when we copied 5\% of subroutines from normal files. With our highest setting of 35\% dead code blocks and 30\% subroutines, most of the scores for viruses and normal files were very close to each other. as shown in Figure 38 of Appendix A.

We also observed that as we increased the amount of dead codes copied, the average scores for viruses and normal files were also closer to each other. In addition, the deviation of normal file scores decreased as more dead codes were copied into virus files. Figure 31 shows the average HMM scores of normal files and family viruses as we increased the percentage of subroutines copied from normal files. With 5\% of subroutines copied, the average score of normal files is -6.15 , and the average score of family viruses is -2.83 . As we increased to $30 \%$ subroutines copied, the average score of normal files increased to -3.8 , and the average score of family viruses remained almost constant at -2.83 .

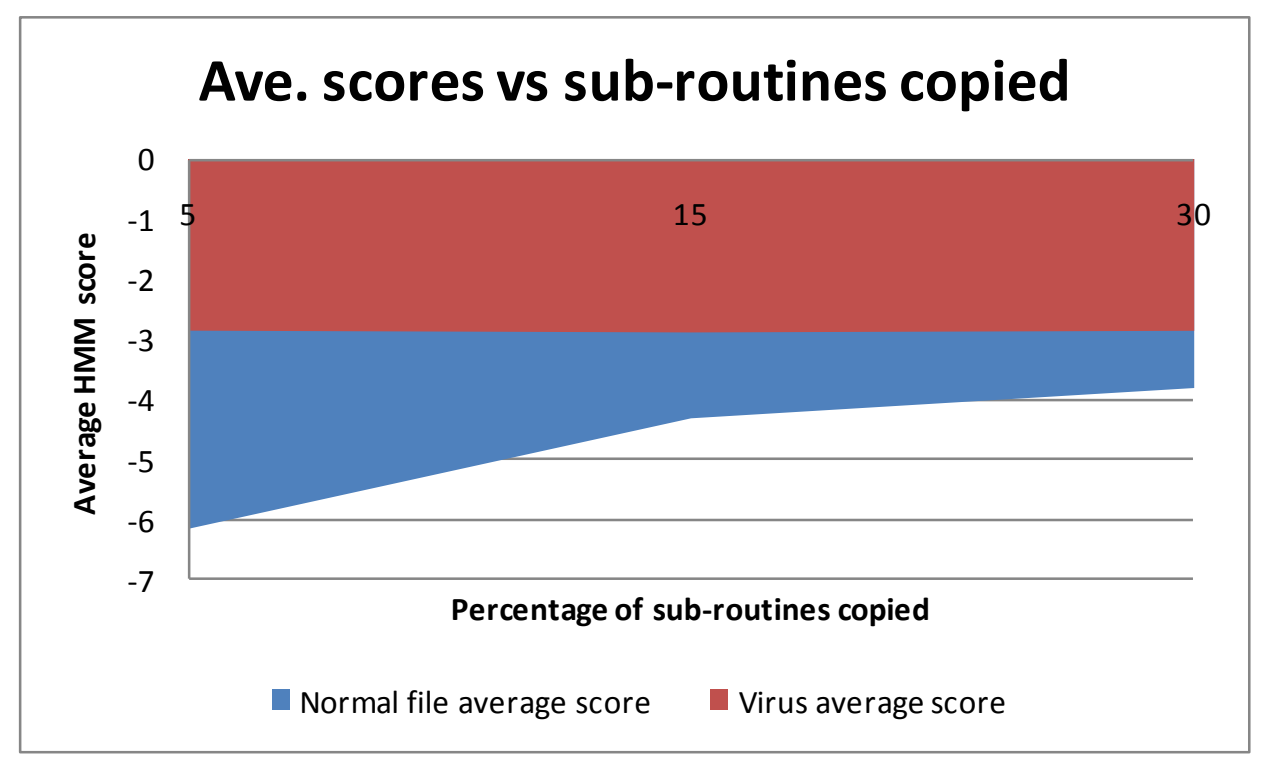

Figure 31. Ave. scores vs. subroutines copied

Figure 32 shows the average HMM scores as we increased the percentage of dead code (not subroutines) copied from normal files. Unlike subroutine copying, more dead code copying did 
not seem to change the average scores of normal files. This might be an indication that HMM favors long identical opcode sequences over short identical opcode sequences.

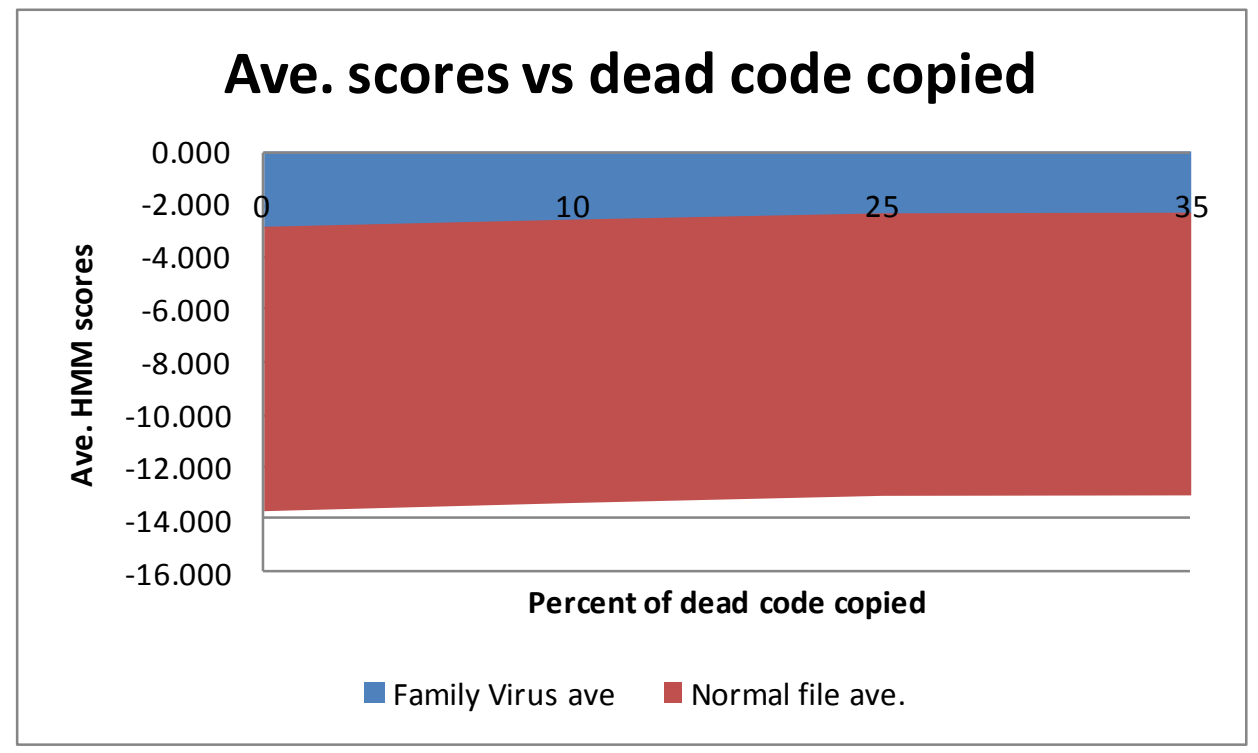

Figure 32. Average scores vs. percent of dead code copied

\section{Future Works}

Based on previous projects [5] and this project, we concluded that in order to escape HMMbased detection, our metamorphic engine must generate viruses that are highly morphed so that similarities of viruses in the same family are low. However, at the same time, we must maintain some similarity between the viruses and normal files. In order to achieve this goal, we copied some codes from normal files. When inserting the copied code into the virus, we simply used unconditional jump instructions to bypass the copied code so that it would not affect the virus operation. These dead codes could easily be detected and removed. More complicated techniques for copying dead codes from normal files are needed in order to prevent virus detectors from detecting and removing dead codes before scanning.

In this project, we showed that the HMM detector was not able to detect all of our viruses. The logical next step would be to enhance the HMM detector. One possible technique would be to first remove dead codes from a virus before computing that virus' HMM score. Another possible 
technique would be to avoid detecting normal files as family viruses by creating more intelligent HMM thresholds. 


\section{References}

[1] M. Stamp, "Information Security: Principles and Practice,” August 2005.

[2] W. Wong, “Analysis and Detection of Metamorphic Computer Viruses," Master's

thesis, San Jose State University, 2006. http://www.cs.sjsu.edu/faculty/stamp/students/Report.pdf [3] S. Attaluri, "Profile hidden Markov models for metamorphic virus analysis," Master's thesis, San Jose State University, 2007.

http://www.cs.sjsu.edu/faculty/stamp/students/Srilatha_cs298Report.pdf

[4] P. Szor, "The Art of Computer Virus Defense and Research," Symantec Press 2005.

[5] P. Desai, “Towards an Undetectable Computer Virus," Master's thesis, San Jose State University, 2008. http://www.cs.sjsu.edu/faculty/stamp/students/Desai_Priti.pdf

[6] Orr, "The Viral Darwinism of W32.Evol: An In-depth Analysis of a Metamorphic eEgine," 2006. http://www.antilife.org/files/Evol.pdf [7] Orr, "The Molecular Virology of Lexotan32: Metamorphism Illustrated," 2007. http://www.antilife.org/files/Lexo32.pdf [8] E. Konstantinou, "Metamorphic Virus: Analysis and Detection,” January 2008.

[9] A. Venkatesan, “Code Obfuscation and Metamorphic Virus Detection,” Master's thesis, San Jose State University, 2008.

http://www.cs.sjsu.edu/faculty/stamp/students/ashwini_venkatesan_cs298report.doc [10] P. Zbitskiy, "Code mutation techniques by means of formal grammars and automatons," Springer-Verlag France 2009. http://www.springerlink.com/content/q0k10h3611827181

[11] P. Mishra, “A Taxonomy of Software Uniqueness Transformations,” December 2003. http://www.cs.sjsu.edu/faculty/stamp/students/FinalReport.doc

[12] J. Aycock, "Computer Viruses and Malware," Springer Science + Business Media, 2006.

[13] E. Daoud and I. Jebril, "Computer Virus Strategies and Detection Methods," Int. J. Open Problems Compt. Math., Vol. 1, No. 2, September 2008.

http://www.emis.de/journals/IJOPCM/files/IJOPCM(vol.1.2.3.S.08).pdf [14] M. Stamp, “A Revealing Introduction to Hidden Markov Models,” January 2004. http://www.cs.sjsu.edu/faculty/stamp/RUA/HMM.pdf 
[15] Walenstein, R. Mathur, M. Chouchane, R. Chouchane, and A. Lakhotia, "The Design Space of Metamorphic Malware," In Proceedings of the 2nd International Conference on Information Warfare, March 2007.

[16] Wikipedia, “Computer Virus,” May 2009, http://en.wikipedia.org/wiki/Computer_virus [17] F. Cohen, "Computer viruses: theory and experiments," Computer Security, 6(1):22-35, 1987.

[18] "Benny/29A," Theme: metamorphism, http://www.vx.netlux.org/lib/static/vdat/epmetam2.htm

[19] J. Borello and L. Me, "Code Obfuscation Techniques for Metamorphic Viruses," Feb 2008, http://www.springerlink.com/content/233883w3r2652537

[20] A. Lakhotia, “Are Metamorphic Viruses Really Invincible?" Virus Bulletin, December 2005.

[21] FASM, http://flatassembler.net/

[22] IDA Pro, http://www.hex-rays.com/idapro/

[23] Wikipedia, "Heuristic analysis," March 2009, http://en.wikipedia.org/wiki/Heuristic_analysis

[24] HowStuffWorks, "Computer \& Internet Security," May 2008, http://computer.howstuffworks.com/virus.htm 


\section{Appendix A: Additional HMM results}

This section contains the HMM results (with state $\mathrm{N}=3$ ) for different amounts of dead code block and dead functions copied from normal files.

\begin{tabular}{|c|c|c|c|c|c|c|c|}
\hline \multicolumn{8}{|c|}{$10 \%$ dead code copying model with $\mathrm{N}=3$} \\
\hline \multicolumn{4}{|c|}{ Family Viruses } & \multicolumn{4}{|c|}{ Normal Files } \\
\hline IDAN120 & -2.794 & IDAN140 & -2.299 & IDARO & -8.588 & IDAR20 & -12.797 \\
\hline IDAN121 & -2.173 & IDAN141 & -2.426 & IDAR1 & -5.966 & IDAR21 & -8.513 \\
\hline IDAN122 & -2.281 & IDAN142 & -2.790 & IDAR2 & -13.791 & IDAR22 & -15.138 \\
\hline IDAN123 & -2.924 & IDAN143 & -2.540 & IDAR3 & -18.344 & IDAR23 & -16.552 \\
\hline IDAN124 & -2.411 & IDAN144 & -2.565 & IDAR4 & -4.949 & IDAR24 & -8.505 \\
\hline IDAN125 & -2.522 & IDAN145 & -2.574 & IDAR5 & -9.099 & IDAR25 & -8.360 \\
\hline IDAN126 & -2.263 & IDAN146 & -2.502 & IDAR6 & -12.520 & IDAR26 & -8.175 \\
\hline IDAN127 & -2.621 & IDAN147 & -2.639 & IDAR7 & -12.485 & IDAR27 & -8.606 \\
\hline IDAN128 & -2.658 & IDAN148 & -2.479 & IDAR8 & -8.165 & IDAR28 & -8.160 \\
\hline IDAN129 & -2.669 & IDAN149 & -3.526 & IDAR9 & -9.174 & IDAR29 & -8.442 \\
\hline IDAN130 & -3.388 & IDAN150 & -2.617 & IDAR10 & -10.509 & IDAR30 & -10.659 \\
\hline IDAN131 & -2.255 & IDAN151 & -2.647 & IDAR11 & -6.220 & IDAR31 & -8.569 \\
\hline IDAN132 & -2.617 & IDAN152 & -2.425 & IDAR12 & -6.220 & IDAR32 & -8.127 \\
\hline IDAN133 & -2.597 & IDAN153 & -3.493 & IDAR13 & -14.365 & IDAR33 & -9.426 \\
\hline IDAN134 & -2.288 & IDAN154 & -2.413 & IDAR14 & -11.565 & IDAR34 & -8.368 \\
\hline IDAN135 & -2.372 & IDAN155 & -2.594 & IDAR15 & -8.438 & IDAR35 & -8.704 \\
\hline IDAN136 & -2.300 & IDAN156 & -2.622 & IDAR16 & -16.226 & IDAR36 & -8.482 \\
\hline IDAN137 & -2.378 & IDAN157 & -2.526 & IDAR17 & -11.811 & IDAR37 & -11.299 \\
\hline IDAN138 & -3.642 & IDAN158 & -2.558 & IDAR18 & -12.528 & IDAR38 & -11.062 \\
\hline IDAN139 & -2.737 & IDAN159 & -2.439 & IDAR19 & -15.952 & IDAR39 & -23.438 \\
\hline \multicolumn{4}{|c|}{ Min Score $=-3.642$} & \multicolumn{4}{|c|}{ Max Score $=-4.949$} \\
\hline \multicolumn{4}{|c|}{0 viruses with scores $<-4.949$} & \multicolumn{4}{|c|}{0 normal files with scores $>-3.642$} \\
\hline
\end{tabular}

Table 15. HMM results with $10 \%$ dead code copied 


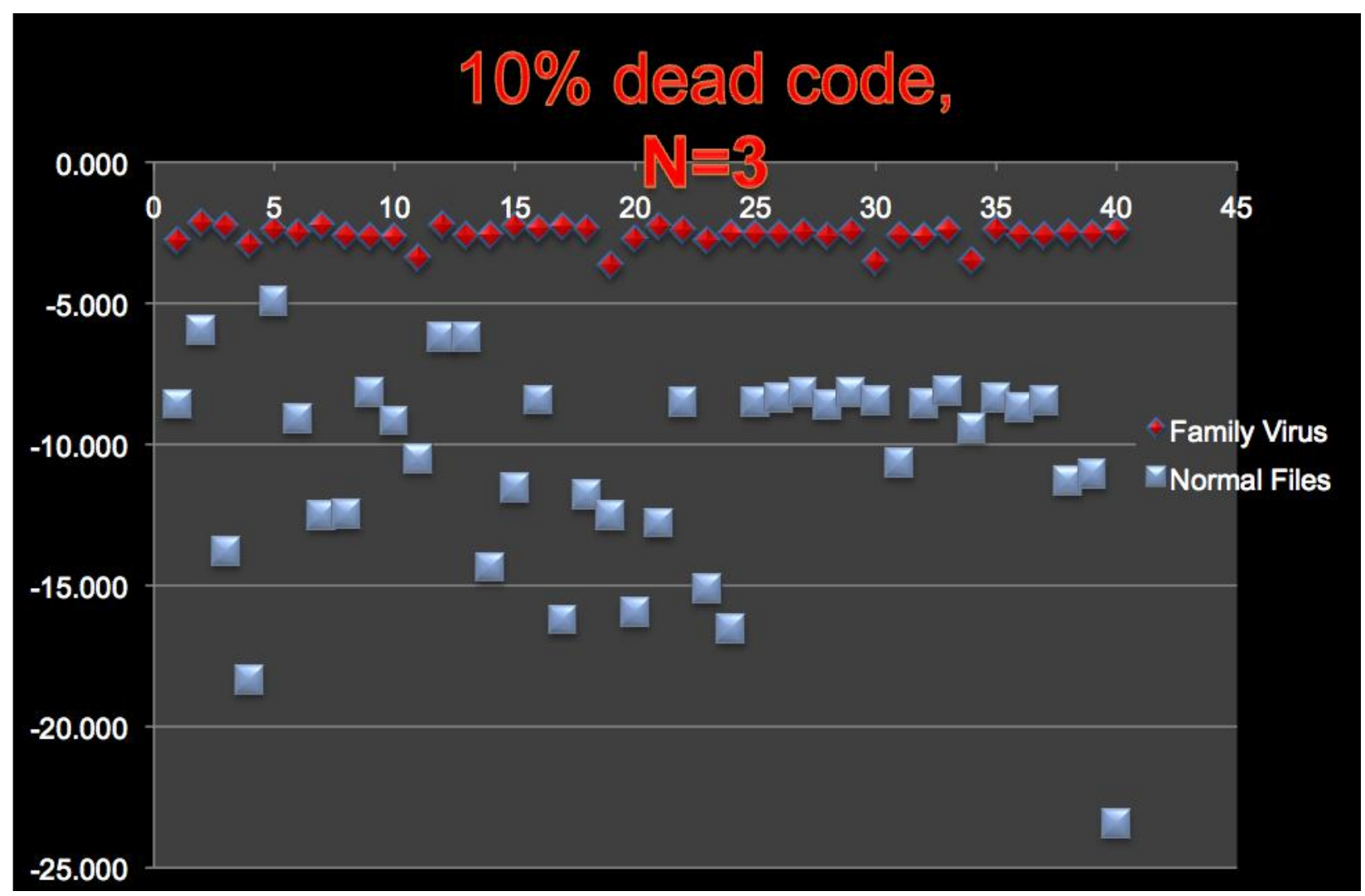

Figure 33. HMM results with $10 \%$ dead code copied

\begin{tabular}{|ll|ll|ll|lr|}
\hline \multicolumn{7}{|c|}{ 25\% dead code copying model with N=3 } \\
\hline \multicolumn{7}{|c|}{ Family Viruses } & \multicolumn{4}{c|}{ Normal Files } \\
\hline IDAN120 & -2.459 & IDAN140 & -2.054 & IDAR0 & -8.564 & IDAR20 & -12.830 \\
IDAN121 & -1.915 & IDAN141 & -2.140 & IDAR1 & -5.949 & IDAR21 & -8.501 \\
IDAN122 & -2.023 & IDAN142 & -2.404 & IDAR2 & -13.793 & IDAR22 & -14.762 \\
IDAN123 & -2.589 & IDAN143 & -2.478 & IDAR3 & -18.363 & IDAR23 & -16.202 \\
IDAN124 & -2.197 & IDAN144 & -2.390 & IDAR4 & -4.919 & IDAR24 & -8.492 \\
IDAN125 & -2.180 & IDAN145 & -2.468 & IDAR5 & -9.133 & IDAR25 & -8.347 \\
IDAN126 & -2.083 & IDAN146 & -2.403 & IDAR6 & -12.549 & IDAR26 & -8.162 \\
IDAN127 & -2.473 & IDAN147 & -2.451 & IDAR7 & -12.514 & IDAR27 & -8.593 \\
IDAN128 & -2.451 & IDAN148 & -2.401 & IDAR8 & -8.203 & IDAR28 & -8.147 \\
IDAN129 & -2.421 & IDAN149 & -2.943 & IDAR9 & -9.181 & IDAR29 & -8.430 \\
IDAN130 & -2.804 & IDAN150 & -2.295 & IDAR10 & -10.538 & IDAR30 & -10.623 \\
IDAN131 & -2.045 & IDAN151 & -2.479 & IDAR11 & -6.213 & IDAR31 & -8.557 \\
IDAN132 & -2.496 & IDAN152 & -2.219 & IDAR12 & -6.213 & IDAR32 & -8.115 \\
IDAN133 & -2.504 & IDAN153 & -3.099 & IDAR13 & -14.397 & IDAR33 & -9.415 \\
IDAN134 & -2.173 & IDAN154 & -2.172 & IDAR14 & -11.555 & IDAR34 & -8.357 \\
IDAN135 & -2.241 & IDAN155 & -2.334 & IDAR15 & -8.473 & IDAR35 & -8.694 \\
\hline
\end{tabular}




\begin{tabular}{|ll|ll|ll|ll|}
\hline IDAN136 & -2.091 & IDAN156 & -2.563 & IDAR16 & -16.212 & IDAR36 & -8.470 \\
IDAN137 & -2.043 & IDAN157 & -2.401 & IDAR17 & -11.631 & IDAR37 & -11.264 \\
IDAN138 & -3.240 & IDAN158 & -2.489 & IDAR18 & -12.530 & IDAR38 & -11.051 \\
IDAN139 & -2.448 & IDAN159 & -2.053 & IDAR19 & -15.586 & IDAR39 & -23.424 \\
\hline \multicolumn{3}{|c|}{ Min Score $=-3.24$} & \multicolumn{3}{c|}{ Max Score $=-4.918$} \\
\hline \multicolumn{3}{|c|}{ viruses with scores <-4.918 } & \multicolumn{3}{c|}{ normal files with scores > -3.24 } \\
\hline
\end{tabular}

Table 16. HMM results with $25 \%$ dead code copied

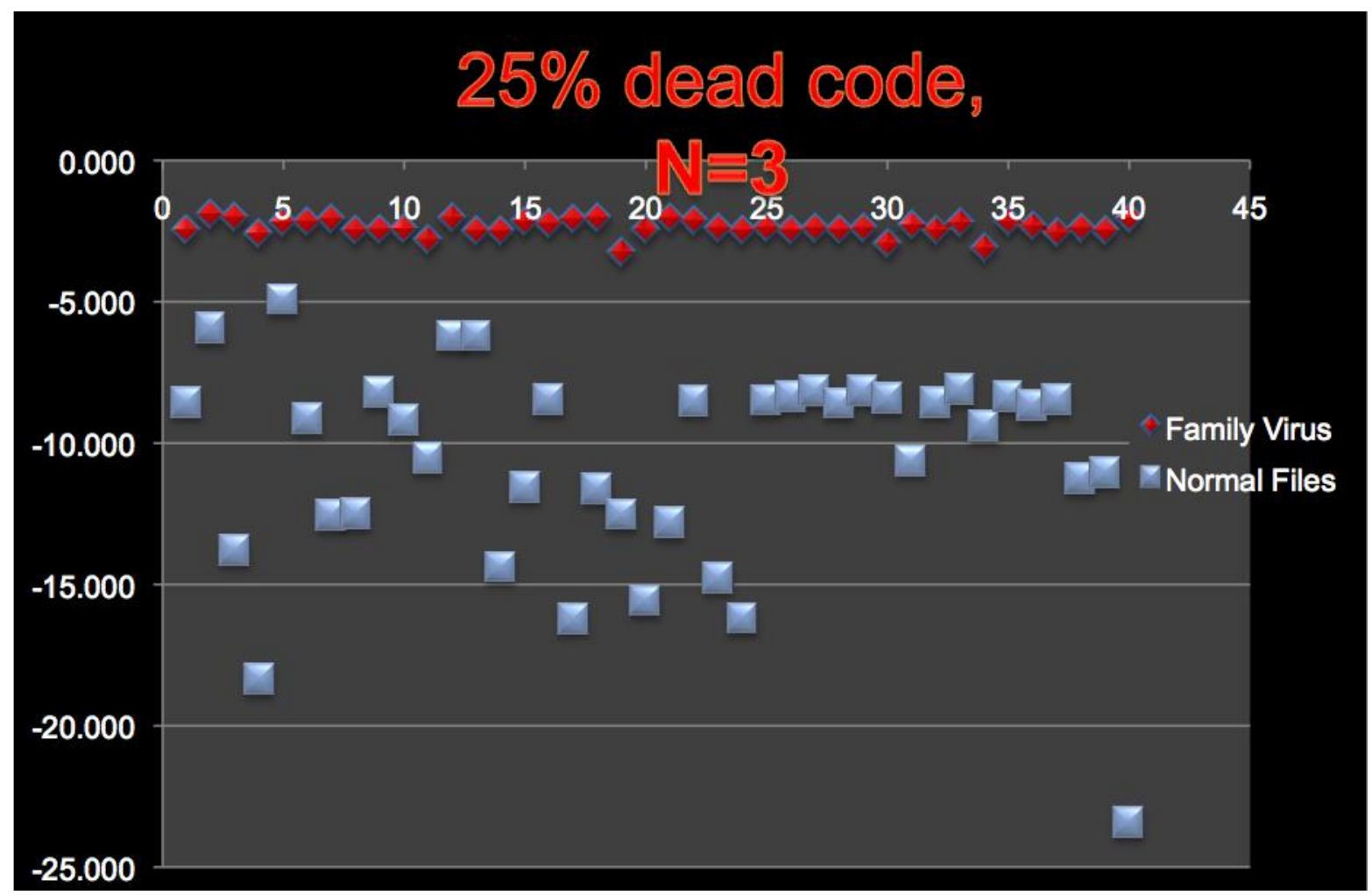

Figure 34. HMM results with $25 \%$ dead code copied

\begin{tabular}{|ll|ll|ll|lr|}
\hline \multicolumn{7}{|c|}{ 35\% dead code copying model with N=3 } \\
\hline \multicolumn{3}{|c|}{ Family Viruses } & \multicolumn{4}{c|}{ Normal Files } \\
\hline IDAN120 & -2.540 & IDAN140 & -2.044 & IDAR0 & -8.529 & IDAR20 & -12.926 \\
IDAN121 & -1.930 & IDAN141 & -2.102 & IDAR1 & -5.906 & IDAR21 & -8.473 \\
IDAN122 & -2.051 & IDAN142 & -2.405 & IDAR2 & -13.798 & IDAR22 & -14.865 \\
IDAN123 & -2.439 & IDAN143 & -2.386 & IDAR3 & -18.345 & IDAR23 & -16.304 \\
IDAN124 & -2.138 & IDAN144 & -2.387 & IDAR4 & -4.889 & IDAR24 & -8.460 \\
IDAN125 & -2.171 & IDAN145 & -2.397 & IDAR5 & -9.212 & IDAR25 & -8.316 \\
IDAN126 & -2.129 & IDAN146 & -2.392 & IDAR6 & -12.640 & IDAR26 & -8.131 \\
IDAN127 & -2.383 & IDAN147 & -2.384 & IDAR7 & -12.589 & IDAR27 & -8.563 \\
\hline
\end{tabular}




\begin{tabular}{|cl|ll|lr|lr|}
\hline IDAN128 & -2.444 & IDAN148 & -2.372 & IDAR8 & -8.299 & IDAR28 & -8.117 \\
IDAN129 & -2.309 & IDAN149 & -2.938 & IDAR9 & -9.151 & IDAR29 & -8.400 \\
IDAN130 & -2.707 & IDAN150 & -2.343 & IDAR10 & -10.643 & IDAR30 & -10.617 \\
IDAN131 & -2.091 & IDAN151 & -2.467 & IDAR11 & -6.190 & IDAR31 & -8.527 \\
IDAN132 & -2.497 & IDAN152 & -2.241 & IDAR12 & -6.190 & IDAR32 & -8.084 \\
IDAN133 & -2.479 & IDAN153 & -2.970 & IDAR13 & -14.500 & IDAR33 & -9.386 \\
IDAN134 & -2.032 & IDAN154 & -2.155 & IDAR14 & -11.527 & IDAR34 & -8.328 \\
IDAN135 & -2.268 & IDAN155 & -2.295 & IDAR15 & -8.563 & IDAR35 & -8.663 \\
IDAN136 & -2.079 & IDAN156 & -2.472 & IDAR16 & -16.196 & IDAR36 & -8.440 \\
IDAN137 & -2.085 & IDAN157 & -2.411 & IDAR17 & -11.622 & IDAR37 & -11.259 \\
IDAN138 & -3.301 & IDAN158 & -2.418 & IDAR18 & -12.509 & IDAR38 & -11.030 \\
IDAN139 & -2.331 & IDAN159 & -2.082 & IDAR19 & -15.686 & IDAR39 & -23.439 \\
\hline \multicolumn{3}{|c|}{ Min Score $=-3.301$} & Max Score = -4.889 \\
\hline \multicolumn{3}{|c|}{0 viruses with scores <-4.889 }
\end{tabular}

Table 17. HMM results with $35 \%$ dead code copied

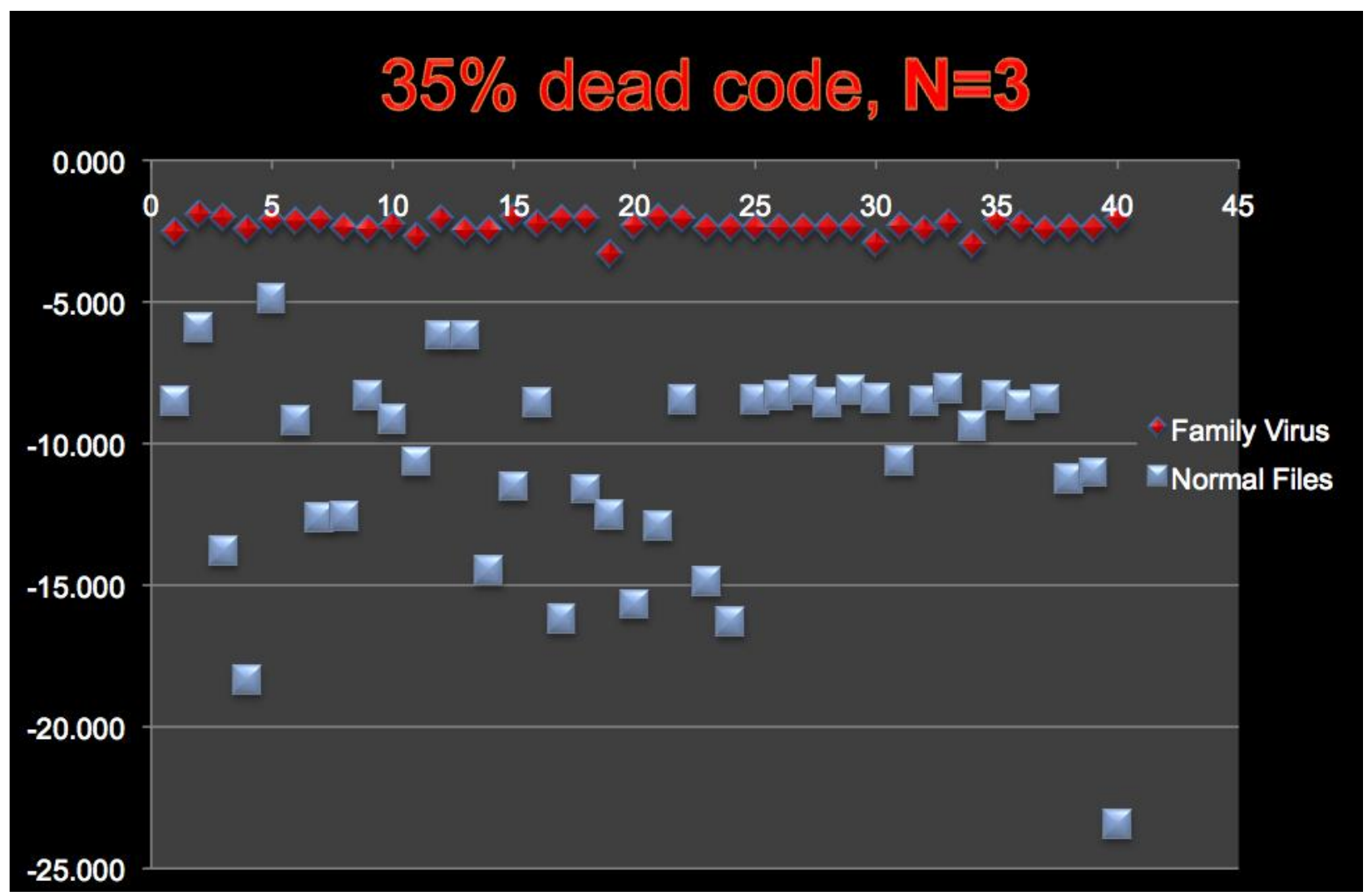

Figure 35. HMM results with $35 \%$ dead code copied 


\begin{tabular}{|c|c|c|c|c|c|c|c|}
\hline \multicolumn{8}{|c|}{$35 \%$ dead code block, and $15 \%$ subroutine copying model with $\mathrm{N}=3$} \\
\hline \multicolumn{4}{|c|}{ Family Viruses } & \multicolumn{4}{|c|}{ Normal Files } \\
\hline IDAN120 & -2.433 & IDAN140 & -1.992 & IDARO & -4.287 & IDAR20 & -3.224 \\
\hline IDAN121 & -1.904 & IDAN141 & -2.103 & IDAR1 & -3.415 & IDAR21 & -2.993 \\
\hline IDAN122 & -1.965 & IDAN142 & -2.361 & IDAR2 & -4.419 & IDAR22 & -8.343 \\
\hline IDAN123 & -2.398 & IDAN143 & -2.464 & IDAR3 & -3.465 & IDAR23 & -8.195 \\
\hline IDAN124 & -2.071 & IDAN144 & -2.372 & IDAR4 & -3.200 & IDAR24 & -2.974 \\
\hline IDAN125 & -2.109 & IDAN145 & -2.400 & IDAR5 & -3.193 & IDAR25 & -2.966 \\
\hline IDAN126 & -2.039 & IDAN146 & -2.351 & IDAR6 & -3.189 & IDAR26 & -2.961 \\
\hline IDAN127 & -2.421 & IDAN147 & -2.361 & IDAR7 & -4.436 & IDAR27 & -2.971 \\
\hline IDAN128 & -2.439 & IDAN148 & -2.353 & IDAR8 & -3.167 & IDAR28 & -2.961 \\
\hline IDAN129 & -2.242 & IDAN149 & -2.879 & IDAR9 & -3.724 & IDAR29 & -2.966 \\
\hline IDAN130 & -4.095 & IDAN150 & -2.341 & IDAR10 & -3.618 & IDAR30 & -3.360 \\
\hline IDAN131 & -2.080 & IDAN151 & -2.412 & IDAR11 & -3.165 & IDAR31 & -2.971 \\
\hline IDAN132 & -2.443 & IDAN152 & -2.100 & IDAR12 & -3.165 & IDAR32 & -2.960 \\
\hline IDAN133 & -2.572 & IDAN153 & -2.893 & IDAR13 & -4.511 & IDAR33 & -2.980 \\
\hline IDAN134 & -2.018 & IDAN154 & -2.636 & IDAR14 & -2.824 & IDAR34 & -2.968 \\
\hline IDAN135 & -2.212 & IDAN155 & -2.247 & IDAR15 & -3.089 & IDAR35 & -2.984 \\
\hline IDAN136 & -2.104 & IDAN156 & -2.435 & IDAR16 & -4.977 & IDAR36 & -2.958 \\
\hline IDAN137 & -2.076 & IDAN157 & -2.486 & IDAR17 & -4.049 & IDAR37 & -3.594 \\
\hline IDAN138 & -2.573 & IDAN158 & -2.363 & IDAR18 & -3.538 & IDAR38 & -3.454 \\
\hline IDAN139 & -2.458 & IDAN159 & -2.085 & IDAR19 & -8.236 & IDAR39 & -6.864 \\
\hline \multicolumn{4}{|c|}{ Min Score $=-4.095$} & \multicolumn{4}{|c|}{ Max Score $=-2.824$} \\
\hline \multicolumn{4}{|c|}{3 viruses with scores $<-2.824$} & \multicolumn{4}{|c|}{31 normal files with scores $>-4.095$} \\
\hline
\end{tabular}

Table 18. HMM results with $35 \%$ dead code blocks and $15 \%$ subroutine copied 


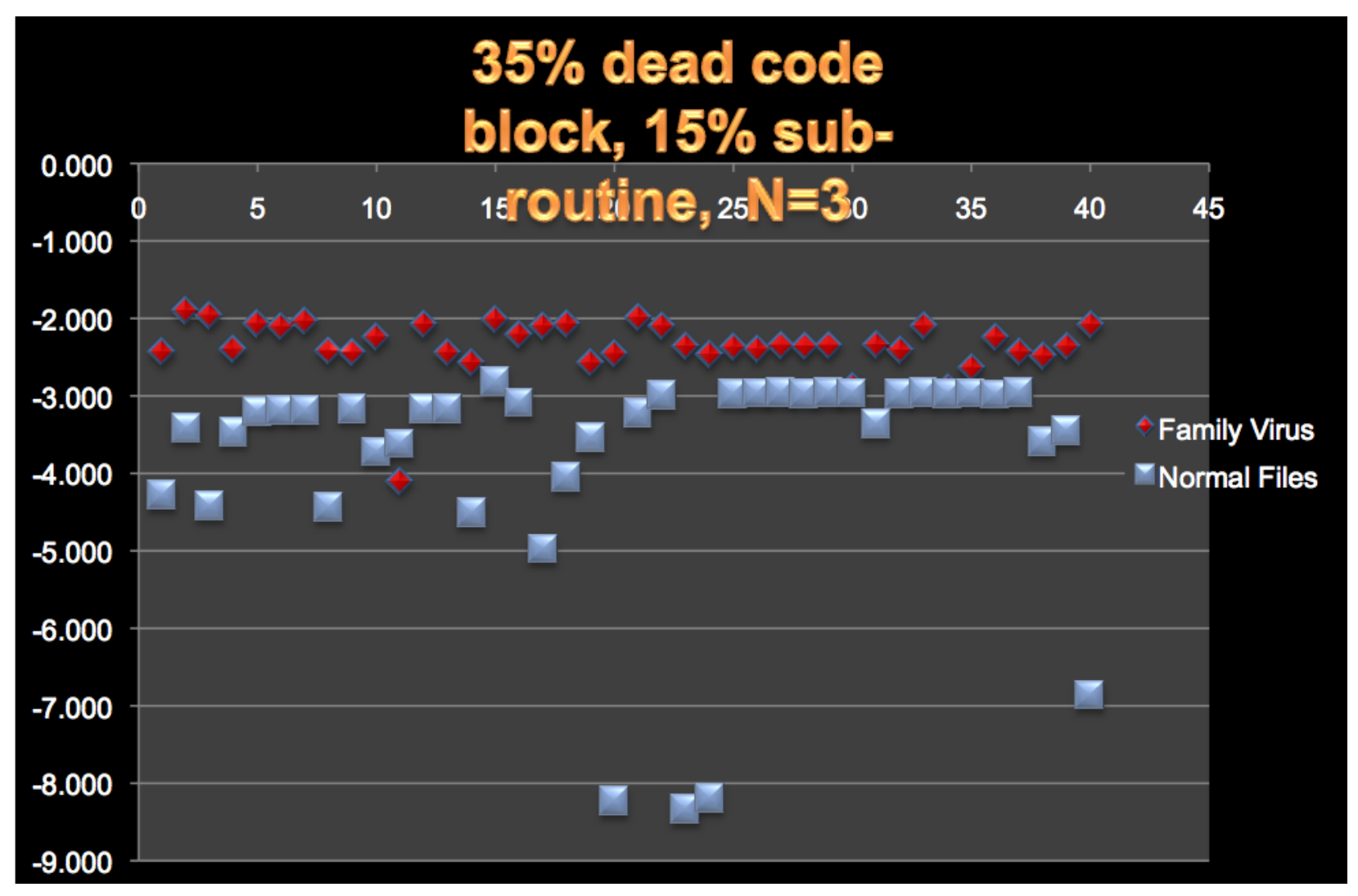

Figure 36. HMM results with $35 \%$ dead code blocks and $15 \%$ subroutines copied

\begin{tabular}{|ll|ll|ll|ll|}
\hline \multicolumn{7}{|c|}{ 35\% dead code block, and 20\% subroutine copying model with N=3 } \\
\hline \multicolumn{3}{|c|}{ Family Viruses } & \multicolumn{4}{c|}{ Normal Files } \\
\hline IDAN120 & -2.336 & IDAN140 & -2.028 & IDAR0 & -3.551 & IDAR20 & -3.028 \\
IDAN121 & -1.881 & IDAN141 & -2.073 & IDAR1 & -3.334 & IDAR21 & -2.884 \\
IDAN122 & -1.989 & IDAN142 & -2.363 & IDAR2 & -4.352 & IDAR22 & -6.927 \\
IDAN123 & -2.431 & IDAN143 & -2.459 & IDAR3 & -3.396 & IDAR23 & -6.878 \\
IDAN124 & -2.077 & IDAN144 & -2.358 & IDAR4 & -3.130 & IDAR24 & -2.865 \\
IDAN125 & -2.198 & IDAN145 & -2.373 & IDAR5 & -3.121 & IDAR25 & -2.858 \\
IDAN126 & -2.068 & IDAN146 & -2.376 & IDAR6 & -3.122 & IDAR26 & -2.853 \\
IDAN127 & -2.415 & IDAN147 & -2.543 & IDAR7 & -4.162 & IDAR27 & -2.863 \\
IDAN128 & -2.449 & IDAN148 & -2.333 & IDAR8 & -3.103 & IDAR28 & -2.853 \\
IDAN129 & -2.220 & IDAN149 & -2.902 & IDAR9 & -2.943 & IDAR29 & -2.859 \\
IDAN130 & -2.664 & IDAN150 & -2.351 & IDAR10 & -3.557 & IDAR30 & -2.263 \\
IDAN131 & -2.093 & IDAN151 & -2.379 & IDAR11 & -3.066 & IDAR31 & -2.861 \\
IDAN132 & -2.565 & IDAN152 & -2.086 & IDAR12 & -3.066 & IDAR32 & -2.852 \\
IDAN133 & -2.531 & IDAN153 & -2.973 & IDAR13 & -4.282 & IDAR33 & -2.871 \\
IDAN134 & -1.985 & IDAN154 & -2.143 & IDAR14 & -2.718 & IDAR34 & -2.862 \\
IDAN135 & -2.230 & IDAN155 & -2.355 & IDAR15 & -3.025 & IDAR35 & -2.875 \\
IDAN136 & -2.071 & IDAN156 & -2.396 & IDAR16 & -4.178 & IDAR36 & -2.853 \\
\hline
\end{tabular}




\begin{tabular}{|ll|cl|cl|cc|}
\hline IDAN137 & -2.046 & IDAN157 & -2.513 & IDAR17 & -3.953 & IDAR37 & -2.259 \\
IDAN138 & -2.544 & IDAN158 & -2.434 & IDAR18 & -2.874 & IDAR38 & -3.357 \\
IDAN139 & -2.380 & IDAN159 & -2.080 & IDAR19 & -6.850 & IDAR39 & -6.793 \\
\hline \multicolumn{3}{|c|}{ Min Score $=-2.973$} & \multicolumn{3}{c|}{ Max Score $=-2.2594$} \\
\hline \multicolumn{3}{|c|}{ 16 viruses with scores $<-2.2594$} & \multicolumn{3}{|c|}{ normal files with scores $>-2.973$} \\
\hline
\end{tabular}

Table 19. HMM results with $35 \%$ dead code blocks and $20 \%$ subroutines copied

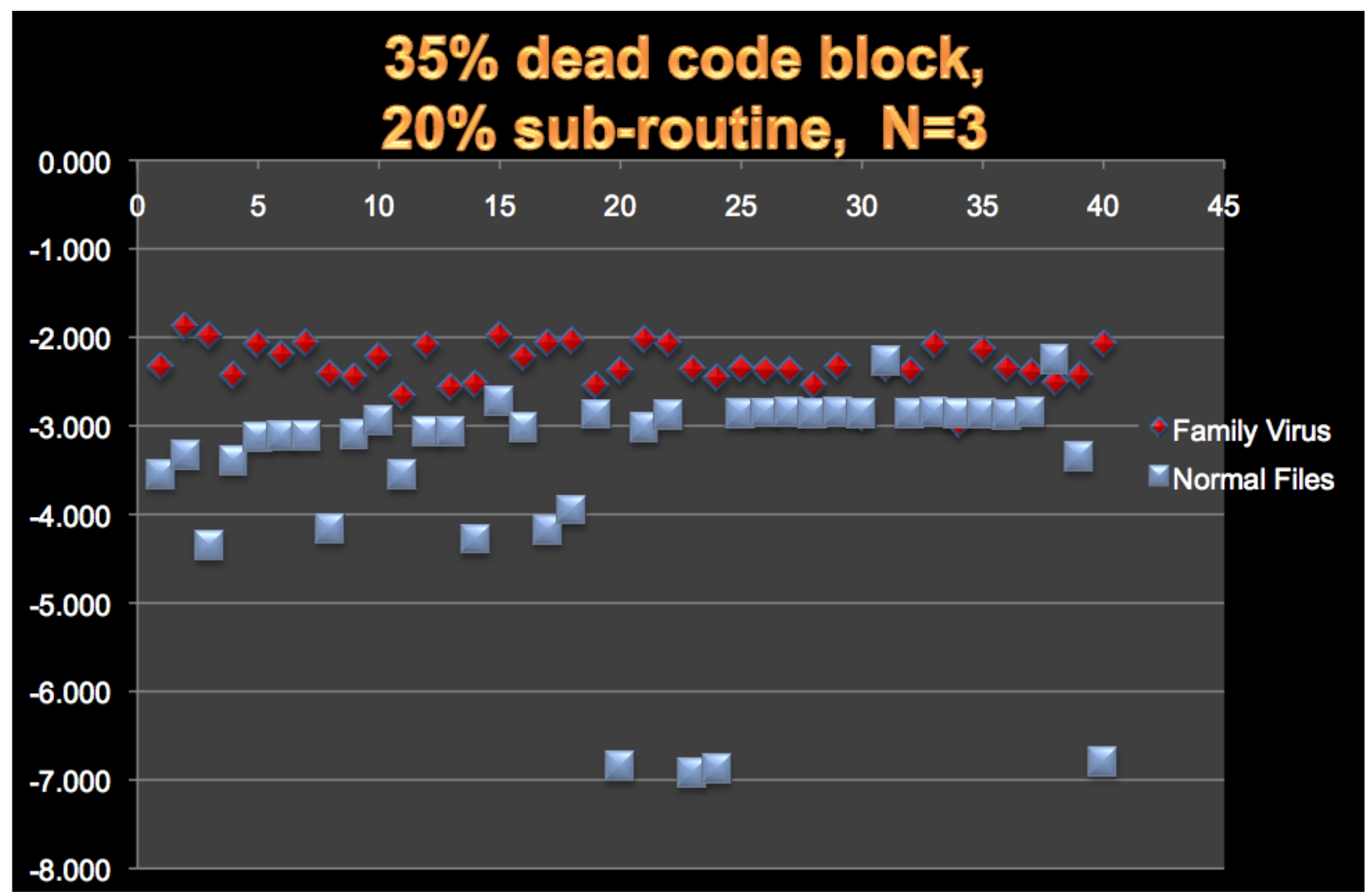

Figure 37. HMM results with $35 \%$ dead code blocks and $20 \%$ subroutines copied

\begin{tabular}{|ll|ll|ll|ll|}
\hline \multicolumn{3}{|c|}{ 35\% dead code block, and 30\% subroutine copying model with N=3 } \\
\hline \multicolumn{3}{|c|}{ Family Viruses } & \multicolumn{4}{c|}{ Normal Files } \\
\hline IDAN120 & -2.294 & IDAN140 & -2.029 & IDAR0 & -2.856 & IDAR20 & -2.849 \\
IDAN121 & -1.891 & IDAN141 & -2.101 & IDAR1 & -2.727 & IDAR21 & -2.708 \\
IDAN122 & -2.043 & IDAN142 & -2.364 & IDAR2 & -3.754 & IDAR22 & -6.352 \\
IDAN123 & -2.520 & IDAN143 & -2.437 & IDAR3 & -2.971 & IDAR23 & -6.339 \\
IDAN124 & -2.100 & IDAN144 & -2.482 & IDAR4 & -2.595 & IDAR24 & -2.693 \\
IDAN125 & -2.455 & IDAN145 & -2.429 & IDAR5 & -2.880 & IDAR25 & -2.686 \\
IDAN126 & -2.088 & IDAN146 & -2.388 & IDAR6 & -2.885 & IDAR26 & -2.682 \\
\hline
\end{tabular}




\begin{tabular}{|c|c|c|c|c|c|c|c|}
\hline IDAN127 & -2.443 & IDAN147 & -2.487 & IDAR7 & -3.922 & IDAR27 & -2.691 \\
\hline IDAN128 & -2.479 & IDAN148 & -2.317 & IDAR8 & -2.881 & IDAR28 & -2.682 \\
\hline IDAN129 & -2.256 & IDAN149 & -2.813 & IDAR9 & -2.692 & IDAR29 & -2.689 \\
\hline IDAN130 & -2.708 & IDAN150 & -2.324 & IDAR10 & -3.351 & IDAR30 & -2.119 \\
\hline IDAN131 & -2.102 & IDAN151 & -2.464 & IDAR11 & -2.853 & IDAR31 & -2.691 \\
\hline IDAN132 & -2.449 & IDAN152 & -2.153 & IDAR12 & -2.853 & IDAR32 & -2.682 \\
\hline IDAN133 & -2.531 & IDAN153 & -2.874 & IDAR13 & -4.071 & IDAR33 & -2.700 \\
\hline IDAN134 & -2.035 & IDAN154 & -2.127 & IDAR14 & -2.508 & IDAR34 & -2.693 \\
\hline IDAN135 & -2.233 & IDAN155 & -3.993 & IDAR15 & -2.828 & IDAR35 & -2.704 \\
\hline IDAN136 & -2.128 & IDAN156 & -2.426 & IDAR16 & -3.978 & IDAR36 & -2.685 \\
\hline IDAN137 & -2.019 & IDAN157 & -2.489 & IDAR17 & -3.762 & IDAR37 & -2.116 \\
\hline IDAN138 & -2.549 & IDAN158 & -2.401 & IDAR18 & -2.659 & IDAR38 & -3.191 \\
\hline IDAN139 & -2.439 & IDAN159 & -2.103 & IDAR19 & -6.286 & IDAR39 & -6.628 \\
\hline \multicolumn{4}{|c|}{ Min Score $=-3.993$} & \multicolumn{4}{|c|}{ Max Score $=-2.116$} \\
\hline \multicolumn{4}{|c|}{30 viruses with scores $<-2.116$} & \multicolumn{4}{|c|}{35 normal files with scores $>-3.993$} \\
\hline
\end{tabular}

Table 20. HMM results with $35 \%$ dead code blocks and $30 \%$ subroutines copied 


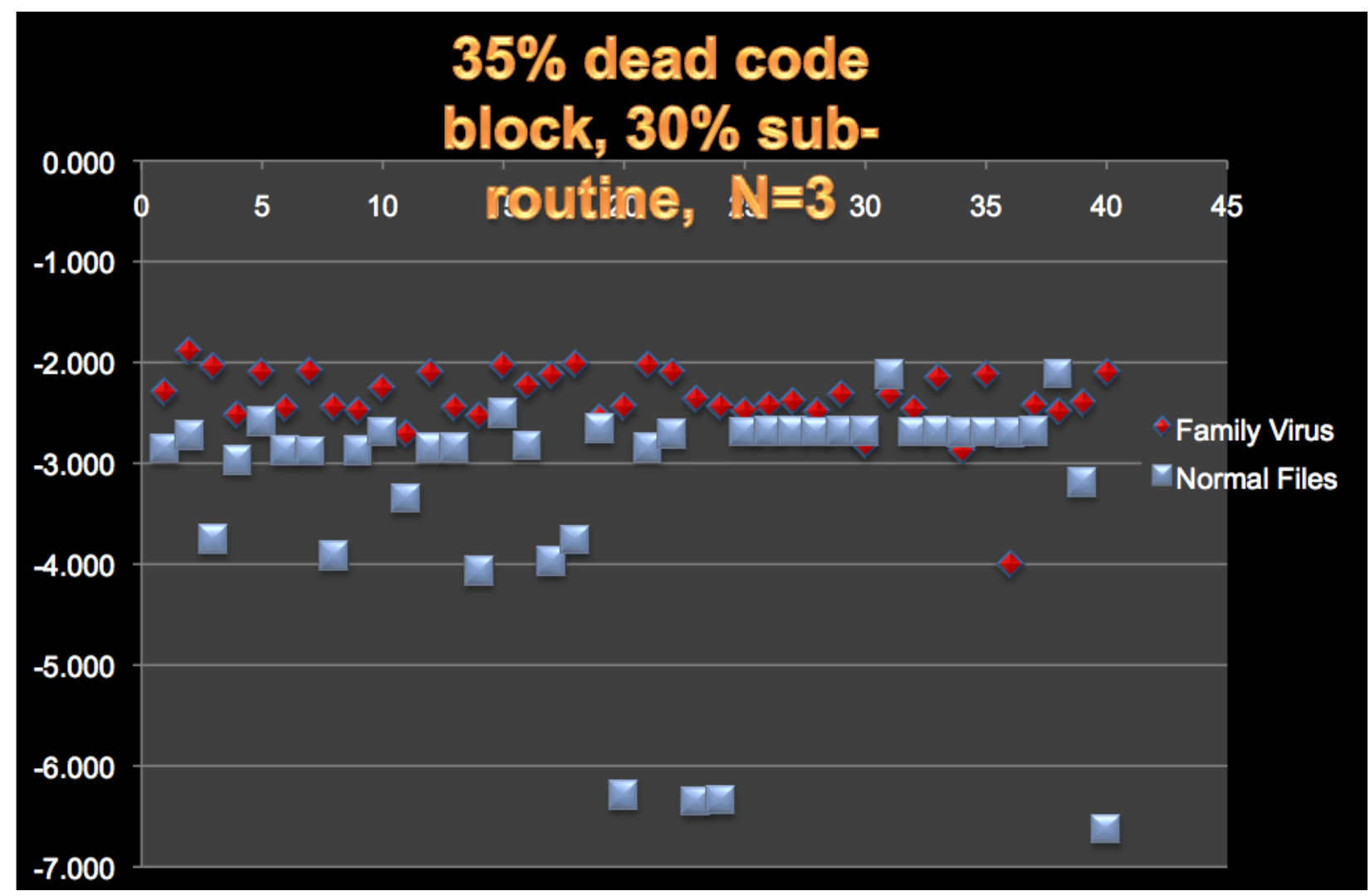

Figure 38. HMM results with $35 \%$ dead code blocks and $30 \%$ subroutines copied

\begin{tabular}{|ll|ll|ll|ll|}
\hline \multicolumn{7}{|c|}{ 15\% subroutine copying model with N=3 } \\
\hline \multicolumn{7}{|c|}{ Family Viruses } & \multicolumn{4}{c|}{ Normal Files } \\
\hline IDAN120 & -4.380 & IDAN140 & -2.445 & IDAR0 & -4.382 & IDAR20 & -2.943 \\
IDAN121 & -2.435 & IDAN141 & -2.740 & IDAR1 & -4.047 & IDAR21 & -3.104 \\
IDAN122 & -2.589 & IDAN142 & -2.735 & IDAR2 & -4.971 & IDAR22 & -14.217 \\
IDAN123 & -4.017 & IDAN143 & -2.606 & IDAR3 & -3.784 & IDAR23 & -13.655 \\
IDAN124 & -2.640 & IDAN144 & -2.706 & IDAR4 & -3.759 & IDAR24 & -3.078 \\
IDAN125 & -2.750 & IDAN145 & -2.624 & IDAR5 & -3.105 & IDAR25 & -3.076 \\
IDAN126 & -2.480 & IDAN146 & -2.505 & IDAR6 & -3.098 & IDAR26 & -3.068 \\
IDAN127 & -3.909 & IDAN147 & -2.689 & IDAR7 & -4.146 & IDAR27 & -3.074 \\
IDAN128 & -2.633 & IDAN148 & -2.632 & IDAR8 & -3.031 & IDAR28 & -3.069 \\
IDAN129 & -2.821 & IDAN149 & -4.411 & IDAR9 & -3.202 & IDAR29 & -3.075 \\
IDAN130 & -4.276 & IDAN150 & -2.804 & IDAR10 & -3.957 & IDAR30 & -2.627 \\
IDAN131 & -2.358 & IDAN151 & -2.624 & IDAR11 & -3.348 & IDAR31 & -3.073 \\
IDAN132 & -2.562 & IDAN152 & -2.642 & IDAR12 & -3.348 & IDAR32 & -3.066 \\
IDAN133 & -2.717 & IDAN153 & -3.834 & IDAR13 & -4.222 & IDAR33 & -3.072 \\
IDAN134 & -2.630 & IDAN154 & -2.441 & IDAR14 & -2.934 & IDAR34 & -3.066 \\
\hline
\end{tabular}




\begin{tabular}{|ll|ll|ll|ll|}
\hline IDAN135 & -2.747 & IDAN155 & -2.746 & IDAR15 & -2.976 & IDAR35 & -3.073 \\
IDAN136 & -2.514 & IDAN156 & -3.204 & IDAR16 & -4.420 & IDAR36 & -3.060 \\
IDAN137 & -2.704 & IDAN157 & -2.596 & IDAR17 & -5.026 & IDAR37 & -2.615 \\
IDAN138 & -2.504 & IDAN158 & -2.581 & IDAR18 & -3.066 & IDAR38 & -3.702 \\
IDAN139 & -2.882 & IDAN159 & -2.524 & IDAR19 & -13.979 & IDAR39 & -7.980 \\
\hline \multicolumn{3}{|c|}{ Min Score $=-4.411$} & \multicolumn{3}{c|}{ Max Score $=-2.615$} \\
\hline \multicolumn{3}{|c|}{ 26 viruses with scores $<-2.615$} & \multicolumn{3}{|c|}{ niles with scores $>-4.411$} \\
\hline
\end{tabular}

\section{Table 21. HMM results with 15\% subroutines copied}

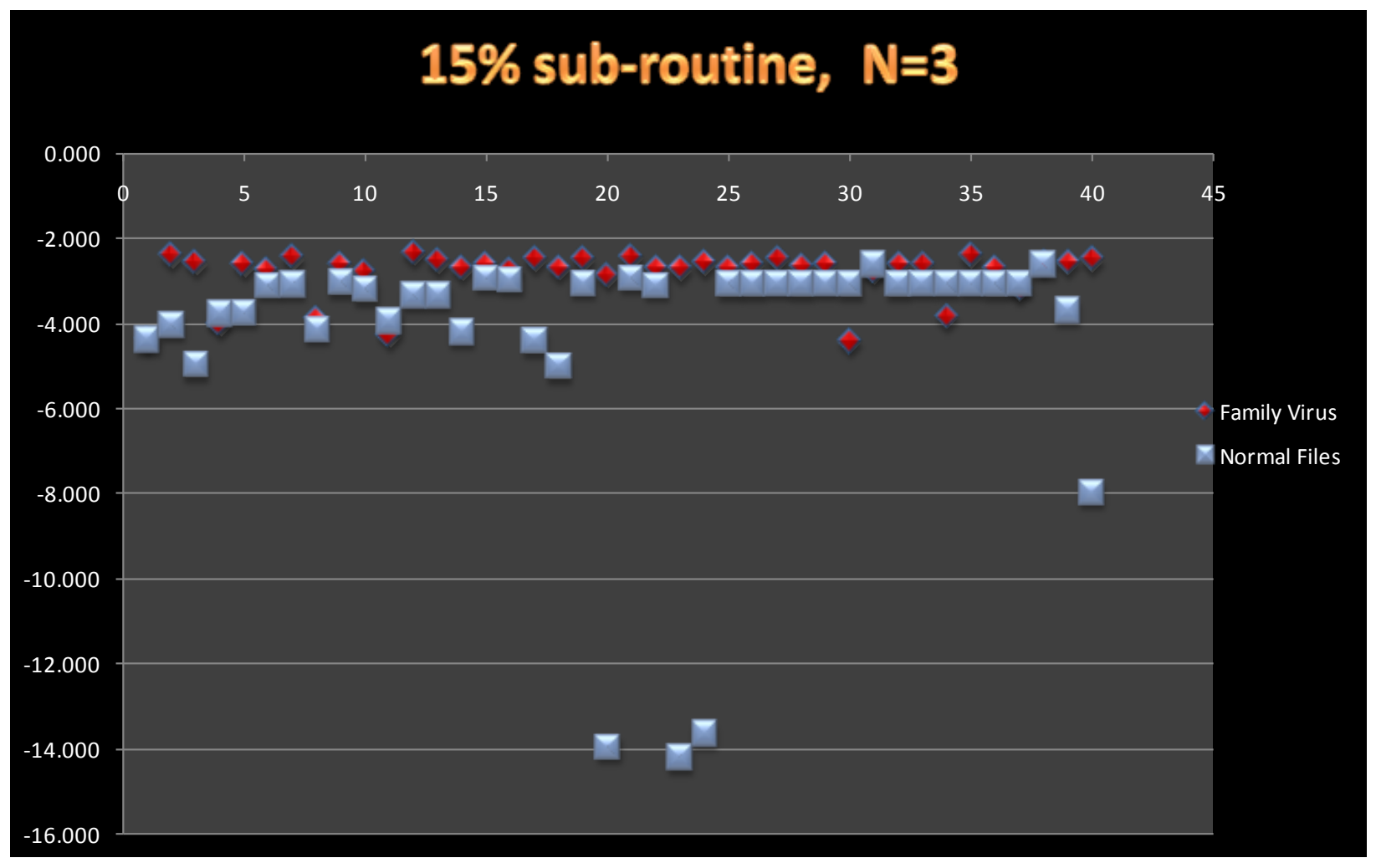

Figure 39. HMM results with $15 \%$ subroutines copied

\begin{tabular}{|ll|ll|ll|ll|}
\hline \multicolumn{7}{|c|}{ 30\% subroutine copying model with N=3 } \\
\hline \multicolumn{3}{|c|}{ Family Viruses } & \multicolumn{4}{c|}{ Normal Files } \\
\hline IDAN120 & -3.415 & IDAN140 & -2.404 & IDAR0 & -4.316 & IDAR20 & -2.901 \\
IDAN121 & -2.206 & IDAN141 & -2.760 & IDAR1 & -3.961 & IDAR21 & -3.030 \\
IDAN122 & -2.641 & IDAN142 & -2.765 & IDAR2 & -5.483 & IDAR22 & -8.602 \\
IDAN123 & -4.149 & IDAN143 & -2.606 & IDAR3 & -3.720 & IDAR23 & -8.422 \\
IDAN124 & -2.502 & IDAN144 & -2.619 & IDAR4 & -3.683 & IDAR24 & -3.003 \\
IDAN125 & -2.711 & IDAN145 & -2.653 & IDAR5 & -3.061 & IDAR25 & -3.001 \\
IDAN126 & -2.427 & IDAN146 & -2.508 & IDAR6 & -3.051 & IDAR26 & -2.994 \\
IDAN127 & -3.693 & IDAN147 & -2.770 & IDAR7 & -4.099 & IDAR27 & -2.999 \\
\hline
\end{tabular}




\begin{tabular}{|c|c|c|c|c|c|c|c|}
\hline IDAN128 & -2.606 & IDAN148 & -2.563 & IDAR8 & -2.991 & IDAR28 & -2.994 \\
\hline IDAN129 & -3.111 & IDAN149 & -3.975 & IDAR9 & -3.123 & IDAR29 & -3.000 \\
\hline IDAN130 & -5.471 & IDAN150 & -2.626 & IDAR10 & -3.573 & IDAR30 & -2.420 \\
\hline IDAN131 & -2.301 & IDAN151 & -2.648 & IDAR11 & -3.280 & IDAR31 & -2.998 \\
\hline IDAN132 & -2.679 & IDAN152 & -2.397 & IDAR12 & -3.280 & IDAR32 & -2.991 \\
\hline IDAN133 & -2.748 & IDAN153 & -3.717 & IDAR13 & -4.179 & IDAR33 & -2.997 \\
\hline IDAN134 & -2.632 & IDAN154 & -2.461 & IDAR14 & -2.855 & IDAR34 & -2.992 \\
\hline IDAN135 & -2.769 & IDAN155 & -2.839 & IDAR15 & -2.935 & IDAR35 & -2.998 \\
\hline IDAN136 & -2.586 & IDAN156 & -2.985 & IDAR16 & -4.698 & IDAR36 & -2.985 \\
\hline IDAN137 & -2.702 & IDAN157 & -2.581 & IDAR17 & -4.285 & IDAR37 & -2.411 \\
\hline IDAN138 & -2.549 & IDAN158 & -2.532 & IDAR18 & -2.989 & IDAR38 & -3.488 \\
\hline IDAN139 & -2.707 & IDAN159 & -2.530 & IDAR19 & -8.480 & IDAR39 & -7.071 \\
\hline \multicolumn{4}{|c|}{ Min Score $=-5.471$} & \multicolumn{4}{|c|}{ Max Score $=-2.411$} \\
\hline \multicolumn{4}{|c|}{36 viruses with scores $<-2.411$} & \multicolumn{4}{|c|}{35 normal files with scores $>-5.471$} \\
\hline
\end{tabular}

Table 22. HMM results with $30 \%$ subroutines copied

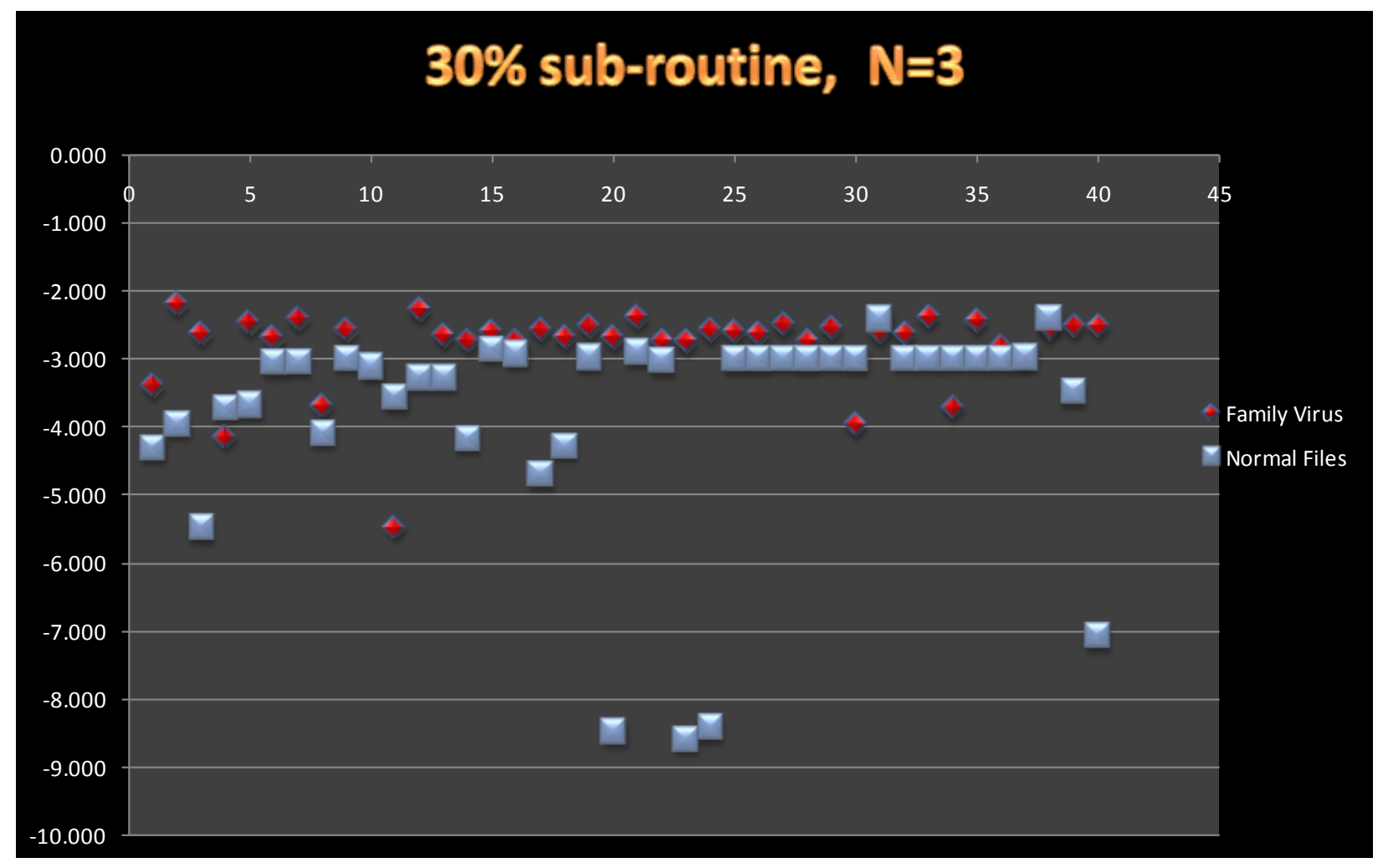

Figure 40. HMM results with $30 \%$ subroutines copied 


\section{Appendix B: Selected HMM models}

Table 23. HMM parameters $(A, B, \pi)$ of the base virus with $N=3$

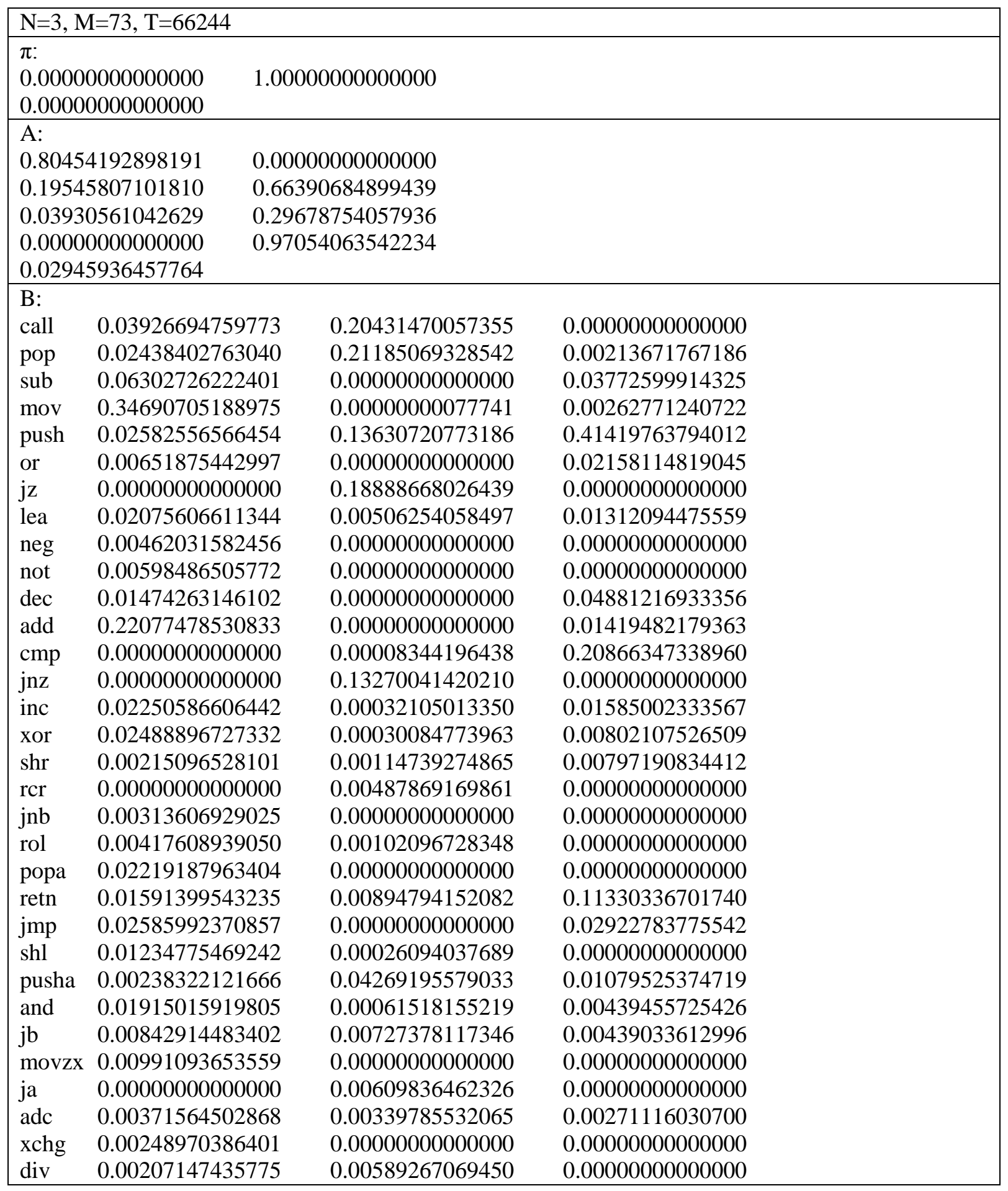




\begin{tabular}{|c|c|c|c|}
\hline imul & 0.00383031363694 & 0.00000000000000 & 0.00000000000000 \\
\hline rep & 0.00126879139224 & 0.00000000000000 & 0.00000000000000 \\
\hline lodsw & 0.00107727571039 & 0.00000000000000 & 0.00000000000000 \\
\hline stosw & 0.00100545732970 & 0.00000000000000 & 0.00000000000000 \\
\hline clc & 0.01149094091083 & 0.00000000000000 & 0.00000000000000 \\
\hline stc & 0.00381651698490 & 0.01305670552198 & 0.00000000000000 \\
\hline test & 0.00000000000000 & 0.00000000000000 & 0.03154629933991 \\
\hline cld & 0.00388283886887 & 0.00307752411317 & 0.00155738131141 \\
\hline start & 0.00020179056938 & 0.00572008164448 & 0.00634401538749 \\
\hline jno & 0.00002393946023 & 0.00000000000000 & 0.00000000000000 \\
\hline $\mathrm{rcl}$ & 0.00548213639288 & 0.00000000000000 & 0.00000000000000 \\
\hline movsb & 0.00270515900609 & 0.00000000000000 & 0.00000000000000 \\
\hline lodsb & 0.00062242596600 & 0.00000000000000 & 0.00000000000000 \\
\hline stosb & 0.00064636542623 & 0.00000000000000 & 0.00000000000000 \\
\hline sar & 0.00140238727500 & 0.00082913628495 & 0.00042902930886 \\
\hline sbb & 0.00115428773412 & 0.00130111848997 & 0.00006419363985 \\
\hline ror & 0.00400373046040 & 0.00282606560572 & 0.00000000000000 \\
\hline jbe & 0.00000000000000 & 0.01024525256707 & 0.00000000000000 \\
\hline bound & 0.00002950068107 & 0.00006242257420 & 0.00000000000000 \\
\hline loop & 0.00045484974439 & 0.00000000000000 & 0.00000000000000 \\
\hline lodsd & 0.00088576002854 & 0.00000000000000 & 0.00000000000000 \\
\hline stosd & 0.00155606491501 & 0.00000000000000 & 0.00000000000000 \\
\hline js & 0.00002393946023 & 0.00000000000000 & 0.00000000000000 \\
\hline in & 0.00001859403840 & 0.00026209056734 & 0.00000000000000 \\
\hline std & 0.00004787892046 & 0.00000000000000 & 0.00000000000000 \\
\hline fld & 0.00002393946023 & 0.00000000000000 & 0.00000000000000 \\
\hline popf & 0.00002393946023 & 0.00000000000000 & 0.00000000000000 \\
\hline jnp & 0.00000000000000 & 0.00008023980862 & 0.00008323461630 \\
\hline ins & 0.00000000000000 & 0.00000000000000 & 0.00008215182120 \\
\hline fnstenv & 0.00000000000000 & 0.00008131152831 & 0.00000000000000 \\
\hline scasb & 0.00002393946023 & 0.00000000000000 & 0.00000000000000 \\
\hline retf & 0.00000000000000 & 0.00016079666922 & 0.00008399708292 \\
\hline $\mathrm{cmc}$ & 0.00002393946023 & 0.00000000000000 & 0.00000000000000 \\
\hline aad & 0.00002393946023 & 0.00000000000000 & 0.00000000000000 \\
\hline enter & 0.00000000000000 & 0.00008131152831 & 0.00000000000000 \\
\hline movsd & 0.00002353094247 & 0.00000000000000 & 0.00008355371070 \\
\hline jp & 0.00002393946023 & 0.00000000000000 & 0.00000000000000 \\
\hline repe & 0.00004787892046 & 0.00000000000000 & 0.00000000000000 \\
\hline jns & 0.00000000000000 & 0.00008131152831 & 0.00000000000000 \\
\hline fild & 0.00002393946023 & 0.00000000000000 & 0.00000000000000 \\
\hline fidiv & 0.00000000000000 & 0.00008131152831 & 0.00000000000000 \\
\hline
\end{tabular}


Table 24. HMM parameters $(A, B, \pi)$ of the virus without dead code copying with $N=3$

\begin{tabular}{|c|c|c|c|c|c|}
\hline \multicolumn{6}{|c|}{$\mathrm{N}=3, \mathrm{M}=87, \mathrm{~T}=136741$} \\
\hline 0.00000000000000 & \\
\hline \multicolumn{6}{|c|}{ 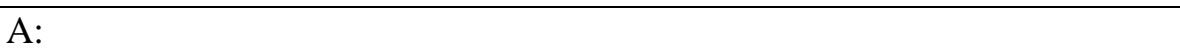 } \\
\hline \multirow{2}{*}{\multicolumn{2}{|c|}{$\begin{array}{l}0.92839338311941 \\
0.03585375542259\end{array}$}} & \multicolumn{2}{|c|}{0.07160661688059} & \multicolumn{2}{|c|}{0.00000000000000} \\
\hline & & \multicolumn{2}{|c|}{0.89850624506005} & \multicolumn{2}{|c|}{0.06563999951736} \\
\hline \multicolumn{2}{|c|}{0.00005823039915} & \multicolumn{2}{|c|}{0.09313151352666} & \multicolumn{2}{|c|}{0.90681025607417} \\
\hline \multicolumn{6}{|l|}{ B: } \\
\hline call & 0.0000000000 & 0000 & 0.00441613 & 2699 & 0.08576566304392 \\
\hline pop & 0.0000000000 & 0000 & 0.05408490 & 2953 & 0.05470811237037 \\
\hline sub & 0.097574056 & 1157 & 0.06099135 & 5007 & 0.04217142357619 \\
\hline $\mathrm{jz}$ & 0.0000000000 & 0000 & 0.00000000 & & 0.05154796043242 \\
\hline lea & 0.0000000000 & 0000 & 0.02442545 & 9823 & 0.02060407599871 \\
\hline mov & 0.1692851368 & 8234 & 0.47381033 & 7281 & 0.11182579930031 \\
\hline neg & 0.0000000000 & 0000 & 0.00045499 & 9038 & 0.00361345332983 \\
\hline jnz & 0.0000000000 & 0000 & 0.00000000 & 0164 & 0.03572404187886 \\
\hline xor & 0.0007041938 & 9245 & 0.01384404 & 8478 & 0.02770564839415 \\
\hline rol & 0.0000000000 & 0000 & 0.00128466 & 9759 & 0.00250427493075 \\
\hline dec & 0.000000000 & 0000 & 0.00673225 & 2317 & 0.02323511407867 \\
\hline $\mathrm{cmp}$ & 0.0007622031 & 6220 & 0.00446994 & 0610 & 0.05457530812780 \\
\hline jmp & 0.0092096125 & 9212 & 0.06712556 & 7858 & 0.03978500846568 \\
\hline push & 0.0000000000 & 0000 & 0.07394660 & 8842 & 0.16762059542512 \\
\hline test & 0.003628046 & 5980 & 0.01030608 & 4306 & 0.01127425058831 \\
\hline add & 0.000000000 & 0000 & 0.12298870 & 4639 & 0.06515733830152 \\
\hline adc & 0.0001607175 & 2857 & 0.00162484 & 2109 & 0.00371605238089 \\
\hline retn & 0.0000000000 & 0000 & 0.00000000 & 0000 & 0.04838775672754 \\
\hline sar & 0.0000000000 & 0000 & 0.00023215 & 5513 & 0.00141081845623 \\
\hline movzx & 0.0002059337 & 5645 & 0.01168520 & 0205 & 0.00476719183819 \\
\hline and & 0.103026549 & 1066 & 0.01360798 & 0885 & 0.01954835478095 \\
\hline or & 0.1037284205 & 1875 & 0.00863662 & 7323 & 0.00322952878411 \\
\hline fldcw & 0.0000000000 & 0000 & 0.00270988 & 9517 & 0.00000000000000 \\
\hline pusha & 0.000000000 & 0000 & 0.00008297 & 4549 & 0.01641601919164 \\
\hline shl & 0.103831415 & 0605 & 0.01015828 & 5549 & 0.00259894510714 \\
\hline popa & 0.0000000000 & 0000 & 0.00000000 & 0000 & 0.02035812386691 \\
\hline $\mathrm{jb}$ & 0.0000000000 & 0000 & 0.00000000 & 0000 & 0.01131261326238 \\
\hline imul & 0.0000000000 & 0000 & 0.00258084 & 6682 & 0.00000000000000 \\
\hline $\mathrm{clc}$ & 0.000000000 & 0000 & 0.00000000 & 0000 & 0.01101491291337 \\
\hline $\mathrm{rcl}$ & 0.0000000000 & 0000 & 0.00007976 & 6229 & 0.00515377097507 \\
\hline rep & 0.0000000000 & 0000 & 0.00139486 & 3262 & 0.00008072016029 \\
\hline shr & 0.100291236 & 7780 & 0.00251187 & 5511 & 0.00269186633729 \\
\hline $\mathrm{sbb}$ & 0.0002796177 & 0664 & 0.00243569 & 8346 & 0.00083147993406 \\
\hline lodsd & 0.0000000000 & 0000 & 0.00011831 & 3882 & 0.00070222725899 \\
\hline stosd & 0.0000000000 & 0000 & 0.00000000 & 0000 & 0.00018320021478 \\
\hline stc & 0.0000000000 & 0000 & 0.00000000 & 0000 & 0.00732800859101 \\
\hline cld & 0.000000000 & 0000 & 0.00210012 & 8363 & 0.00203357265544 \\
\hline inc & 0.0000000000 & 0000 & 0.01129816 & 5698 & 0.01402782855571 \\
\hline div & 0.0000000000 & 0000 & 0.00274214 & 7725 & 0.00000000000000 \\
\hline
\end{tabular}




\begin{tabular}{|c|c|c|c|}
\hline jbe & 0.00000000000000 & 0.00000000000000 & 0.00290830340956 \\
\hline ja & 0.00000000000000 & 0.00000000000000 & 0.00171750201352 \\
\hline jnb & 0.00000000000000 & 0.00000000000000 & 0.00302280354379 \\
\hline ror & 0.00000000000000 & 0.00056090070663 & 0.00389819989411 \\
\hline movsb & 0.00000000000000 & 0.00018853953071 & 0.00232003518421 \\
\hline not & 0.00000000000000 & 0.00139475046273 & 0.00353879197888 \\
\hline start & 0.00000000000000 & 0.00000000000000 & 0.00352660413443 \\
\hline xchg & 0.00000000000000 & 0.00144231485576 & 0.00042556167394 \\
\hline lodsw & 0.00000000000000 & 0.00000000000000 & 0.00103050120811 \\
\hline rer & 0.00000000000000 & 0.00000000000000 & 0.00137400161082 \\
\hline stosw & 0.00000000000000 & 0.00000000000000 & 0.00096180112757 \\
\hline loop & 0.00000000000000 & 0.00010759967769 & 0.00028234223057 \\
\hline movsx & 0.00000000000000 & 0.00072586309692 & 0.00000000000000 \\
\hline repe & 0.00012309451679 & 0.00101902501266 & 0.00000000000000 \\
\hline lodsb & 0.00000000000000 & 0.00000000000000 & 0.00059540069802 \\
\hline repne & 0.00000000000000 & 0.00006452116417 & 0.00000000000000 \\
\hline fld & 0.00023001612580 & 0.00039654791766 & 0.00002903242821 \\
\hline fstp & 0.00045602501776 & 0.00019079257682 & 0.00000000000000 \\
\hline shrd & 0.00000000000000 & 0.00004839087313 & 0.00000000000000 \\
\hline fmul & 0.10584841949022 & 0.00028346239424 & 0.00000000000000 \\
\hline fdiv & 0.09838969982380 & 0.00019947028372 & 0.00000000000000 \\
\hline stosb & 0.00000000000000 & 0.00001170077347 & 0.00014368870672 \\
\hline js & 0.00000000000000 & 0.00000000000000 & 0.00002290002685 \\
\hline in & 0.00000000000000 & 0.00000000000000 & 0.00009160010739 \\
\hline std & 0.00000000000000 & 0.00000000000000 & 0.00004580005369 \\
\hline jno & 0.00000000000000 & 0.00000000000000 & 0.00002290002685 \\
\hline popf & 0.00000000000000 & 0.00009951793682 & 0.00001901548210 \\
\hline bound & 0.00000000000000 & 0.00000000000000 & 0.00004580005369 \\
\hline jnp & 0.00000000000000 & 0.00000000000000 & 0.00004580005369 \\
\hline ins & 0.00000000000000 & 0.00000000000000 & 0.00002290002685 \\
\hline fnstenv & 0.00000000000000 & 0.00001613029104 & 0.00000000000000 \\
\hline scasb & 0.00000000000000 & 0.00001613029104 & 0.00000000000000 \\
\hline retf & 0.00000000000000 & 0.00000000000000 & 0.00006870008054 \\
\hline $\mathrm{cmc}$ & 0.00000000000000 & 0.00000000000000 & 0.00002290002685 \\
\hline fldz & 0.00002121763590 & 0.00002162466282 & 0.00000000000000 \\
\hline fadd & 0.10201907433146 & 0.00002543691095 & 0.00000000000000 \\
\hline leave & 0.00003224337824 & 0.00000000000000 & 0.00002285376964 \\
\hline aad & 0.00000000000000 & 0.00000000000000 & 0.00002290002685 \\
\hline enter & 0.00000000000000 & 0.00000000000000 & 0.00002290002685 \\
\hline movsd & 0.00000000000000 & 0.00000000000000 & 0.00004580005369 \\
\hline jp & 0.00000000000000 & 0.00000000000000 & 0.00002290002685 \\
\hline jns & 0.00000000000000 & 0.00000000000000 & 0.00002290002685 \\
\hline fild & 0.00000000000000 & 0.00000000000000 & 0.00002290002685 \\
\hline fidiv & 0.00000000000000 & 0.00000000000000 & 0.00002290002685 \\
\hline mul & 0.00000000000000 & 0.00020969378355 & 0.00000000000000 \\
\hline fst & 0.00012871351642 & 0.00000000000000 & 0.00000000000000 \\
\hline fdivr & 0.00006435675821 & 0.00000000000000 & 0.00000000000000 \\
\hline cmpsb & 0.00000000000000 & 0.00009678174626 & 0.00000000000000 \\
\hline
\end{tabular}


Table 25. HMM parameters $(A, B, \pi)$ of the virus with most dead code copied with $N=3$

\begin{tabular}{|c|c|c|c|c|c|}
\hline \multicolumn{6}{|c|}{$\mathrm{N}=3, \mathrm{M}=112, \mathrm{~T}=267955$} \\
\hline \multicolumn{2}{|c|}{$\begin{array}{l}\pi: \\
0.00000000000000\end{array}$} & \multicolumn{2}{|c|}{1.00000000000000} & \multicolumn{2}{|c|}{0.00000000000000} \\
\hline \multicolumn{6}{|c|}{ A: } \\
\hline \multicolumn{2}{|c|}{0.90961917297138} & \multicolumn{2}{|c|}{0.08975476506203} & \multicolumn{2}{|c|}{0.00062606196659} \\
\hline \multicolumn{2}{|c|}{0.16278844214058} & \multicolumn{2}{|c|}{0.83662974533219} & \multicolumn{2}{|c|}{0.00058181252720} \\
\hline \multicolumn{2}{|c|}{0.00203565693629} & \multicolumn{2}{|c|}{0.00296737275980} & \multicolumn{2}{|c|}{0.99499697030392} \\
\hline \multicolumn{6}{|l|}{ B: } \\
\hline call & 0.033402420 & 2570 & 0.04112728 & 7600 & 0.00000000000000 \\
\hline pop & 0.000492473 & 1741 & 0.10069227 & 1024 & 0.00205579749002 \\
\hline sub & 0.031988837 & 8658 & 0.04453184 & 9874 & 0.10149690582470 \\
\hline or & 0.005695520 & 9412 & 0.00253280 & 6145 & 0.10358288110428 \\
\hline jmp & 0.068440180 & 6837 & 0.10773195 & 6644 & 0.00117652102598 \\
\hline push & 0.000000000 & 0000 & 0.26050410 & 4581 & 0.00197780928625 \\
\hline mov & 0.606109206 & 3406 & 0.18000930 & 1713 & 0.17679799939390 \\
\hline test & 0.020079722 & 8643 & 0.00110474 & 0929 & 0.00154098572633 \\
\hline lea & 0.025565278 & 0958 & 0.01489172 & 8426 & 0.00000000000000 \\
\hline neg & $0.000299856^{\prime}$ & 6599 & 0.00137469 & 3387 & 0.00000000000000 \\
\hline movzx & 0.015551107 & 3423 & 0.00143847 & 2504 & 0.00000000000000 \\
\hline and & 0.010992069 & 7873 & 0.00990765 & 3504 & 0.10019727020307 \\
\hline fldew & 0.000883293 & 8899 & 0.00044872 & 5468 & 0.00000000000000 \\
\hline jnz & 0.010650894 & 2651 & 0.00457439 & 3072 & 0.00000000000000 \\
\hline xor & 0.010713854 & 1240 & 0.01624638 & 2724 & 0.00099218176261 \\
\hline rol & 0.000719164 & 3324 & 0.00092202 & 7328 & 0.00000000000000 \\
\hline $\mathrm{jz}$ & 0.020337618 & 0840 & 0.00229533 & 0581 & 0.00000000000000 \\
\hline add & 0.039293084 & 0474 & 0.10671411 & 6195 & 0.00183696532896 \\
\hline adc & 0.000895125 & 1402 & 0.00209742 & 5973 & 0.00013729369729 \\
\hline retn & 0.000000000 & 0000 & 0.02598057 & 0550 & 0.00000000000000 \\
\hline sar & 0.000240241 & 7282 & 0.00077685 & 4143 & 0.00000000000000 \\
\hline rep & 0.000695607 & 5696 & 0.00022368 & 2161 & 0.00000000000000 \\
\hline $\operatorname{shr}$ & 0.005058942 & 0636 & 0.00130804 & 1768 & 0.09956814041538 \\
\hline shl & 0.008581224 & 2443 & 0.00309136 & 4802 & 0.10079222536899 \\
\hline $\mathrm{jb}$ & 0.001408968 & 0094 & 0.00401375 & 2847 & 0.00006992784722 \\
\hline imul & 0.000209140 & 8121 & 0.00190338 & 7329 & 0.00000000000000 \\
\hline dec & 0.005904305 & 8669 & 0.00911428 & 1993 & 0.00000000000000 \\
\hline clc & 0.000000000 & 0000 & 0.00565715 & 9101 & 0.00000000000000 \\
\hline $\mathrm{rcl}$ & 0.000000000 & 0000 & 0.00270508 & 0714 & 0.00000000000000 \\
\hline inc & 0.017248036 & 6332 & 0.00521405 & 1870 & 0.00000000000000 \\
\hline $\operatorname{div}$ & 0.000466657 & 9136 & 0.00088479 & 7294 & 0.00000000000000 \\
\hline sbb & 0.000689208 & 2227 & 0.00158769 & 8981 & 0.00024058451061 \\
\hline lodsd & 0.000021613 & 9984 & 0.00040783 & 6498 & 0.00000000000000 \\
\hline stosd & 0.000000000 & 0000 & 0.00009408 & 9590 & 0.00000000000000 \\
\hline stc & 0.000000000 & 0000 & 0.00376359 & 3602 & 0.00000000000000 \\
\hline cld & 0.001307756 & 5529 & 0.00099820 & 4519 & 0.00000000000000 \\
\hline popa & 0.000000000 & 0000 & 0.00815053 & 3237 & 0.00000000000000 \\
\hline pusha & 0.000200056 & 5856 & 0.00643612 & 0604 & 0.00000000000000 \\
\hline $\mathrm{cmp}$ & 0.039130699 & 2941 & 0.00202135 & 1526 & 0.00000000000000 \\
\hline
\end{tabular}




\begin{tabular}{|c|c|c|c|}
\hline jbe & 0.00117038365878 & 0.00000000000000 & 0.00000000000000 \\
\hline ja & 0.00090379626984 & 0.00000000000000 & 0.00000000000000 \\
\hline jnb & 0.00046571010328 & 0.00166275403349 & 0.00000000000000 \\
\hline ror & 0.00018295571818 & 0.00208011816837 & 0.00000000000000 \\
\hline not & 0.00072067122422 & 0.00126037942031 & 0.00000000000000 \\
\hline jle & 0.00135894547048 & 0.00000000000000 & 0.00000000000000 \\
\hline jg & 0.00048115772639 & 0.00000000000000 & 0.00000000000000 \\
\hline jl & 0.00056568543508 & 0.00000000000000 & 0.00000000000000 \\
\hline js & 0.00014569740258 & 0.00000696691874 & 0.00000000000000 \\
\hline movsb & 0.00027918621954 & 0.00082401994636 & 0.00000000000000 \\
\hline start & 0.00000000000000 & 0.00181123049608 & 0.00000000000000 \\
\hline xchg & 0.00014639238523 & 0.00061729413806 & 0.00000000000000 \\
\hline lodsw & 0.00000000000000 & 0.00052925566444 & 0.00000000000000 \\
\hline $\mathrm{rcr}$ & 0.00000000000000 & 0.00070567421925 & 0.00000000000000 \\
\hline stosw & 0.00000000000000 & 0.00049397195348 & 0.00000000000000 \\
\hline loop & 0.00000000000000 & 0.00022346350276 & 0.00000000000000 \\
\hline setnz & 0.00040313214914 & 0.00000000000000 & 0.00000000000000 \\
\hline movsx & 0.00361406683318 & 0.00177796950003 & 0.00000000000000 \\
\hline repe & 0.00141096252198 & 0.00000000000000 & 0.00000000000000 \\
\hline jge & 0.00051366838358 & 0.00000000000000 & 0.00000000000000 \\
\hline setz & 0.00013076523961 & 0.00003397663227 & 0.00000000000000 \\
\hline jns & 0.00005201705150 & 0.00000000000000 & 0.00000000000000 \\
\hline$c d q$ & 0.00013654476019 & 0.00000000000000 & 0.00000000000000 \\
\hline jp & 0.00002629541291 & 0.00001124230748 & 0.00000000000000 \\
\hline lodsb & 0.00000000000000 & 0.00030579216168 & 0.00000000000000 \\
\hline leave & 0.00000000000000 & 0.00027050845071 & 0.00000000000000 \\
\hline outsd & 0.00000000000000 & 0.00004704494795 & 0.00000000000000 \\
\hline shld & 0.00003251065719 & 0.00000000000000 & 0.00000000000000 \\
\hline outsb & 0.00000000000000 & 0.00012937360686 & 0.00000000000000 \\
\hline repne & 0.00005201705150 & 0.00000000000000 & 0.00000000000000 \\
\hline fstp & 0.00017621498694 & 0.00034822065477 & 0.00031892825968 \\
\hline fst & 0.00006429696744 & 0.00000120624953 & 0.00003462852496 \\
\hline fld & 0.00013824276517 & 0.00045017479467 & 0.00008453276497 \\
\hline shrd & 0.00003901278863 & 0.00000000000000 & 0.00000000000000 \\
\hline mul & 0.00024057886319 & 0.00000000000000 & 0.00000000000000 \\
\hline stosb & 0.00000000000000 & 0.00008232865891 & 0.00000000000000 \\
\hline in & 0.00000000000000 & 0.00004704494795 & 0.00000000000000 \\
\hline std & 0.00000000000000 & 0.00002352247398 & 0.00000000000000 \\
\hline jno & 0.00000000000000 & 0.00001176123699 & 0.00000000000000 \\
\hline idiv & 0.00004551492006 & 0.00000000000000 & 0.00000000000000 \\
\hline setl & 0.00001905485471 & 0.00001257799443 & 0.00000000000000 \\
\hline popf & 0.00000000000000 & 0.00008232865891 & 0.00000000000000 \\
\hline bound & 0.00000000000000 & 0.00002352247398 & 0.00000000000000 \\
\hline jnp & 0.00000000000000 & 0.00001583199924 & 0.00002244459866 \\
\hline ins & 0.00000000000000 & 0.00000000000000 & 0.00003432509083 \\
\hline fnstenv & 0.00000000000000 & 0.00000000000000 & 0.00003432509083 \\
\hline scasb & 0.00000000000000 & 0.00000000000000 & 0.00003432509083 \\
\hline retf & 0.00000000000000 & 0.00003528371096 & 0.00000000000000 \\
\hline $\mathrm{cmc}$ & 0.00000650213144 & 0.00000000000000 & 0.00000000000000 \\
\hline fldz & 0.00001565117786 & 0.00004488848794 & 0.00006097059344 \\
\hline fadd & 0.00099439591424 & 0.00073438598041 & 0.10416377678639 \\
\hline
\end{tabular}




\begin{tabular}{|llll|}
\hline fmul & 0.00104298879866 & 0.00074563300951 & 0.10263872476798 \\
jo & 0.00000000000000 & 0.00001176123699 & 0.00000000000000 \\
insd & 0.00000000000000 & 0.00003528371096 & 0.00000000000000 \\
outsw & 0.00000000000000 & 0.00001176123699 & 0.00000000000000 \\
arpl & 0.00000000000000 & 0.00001176123699 & 0.00000000000000 \\
aad & 0.00000000000000 & 0.00001176123699 & 0.00000000000000 \\
enter & 0.00000000000000 & 0.00001176123699 & 0.00000000000000 \\
movsd & 0.00000000000000 & 0.00002352247398 & 0.00000000000000 \\
fdivr & 0.00001950639431 & 0.00000000000000 & 0.00000000000000 \\
fdiv & 0.00111380342971 & 0.00081453028268 & 0.09945510750901 \\
fild & 0.00000000000000 & 0.00004704494795 & 0.00000000000000 \\
fidiv & 0.00000000000000 & 0.00001176123699 & 0.00000000000000 \\
fxch & 0.00000000000000 & 0.00001099193210 & 0.00010522048362 \\
fucompp & 0.00000000000000 & 0.000000000000000 & 0.000010297527248 \\
fnstsw & 0.00000000000000 & 0.00000000000000 & 0.00017162545414 \\
sahf & 0.00000000000000 & 0.00000000000000 & 0.00017162545414 \\
fnstcw & 0.00000650213144 & 0.00000000000000 & 0.00000000000000 \\
fist & 0.00001300426288 & 0.00000000000000 & 0.00000000000000 \\
fsub & 0.00000000000000 & 0.00000000000000 & 0.00003432509083 \\
fucom & 0.00000000000000 & 0.00000000000000 & 0.00003432509083 \\
fucomp & 0.00000000000000 & 0.00000000000000 & 0.00003432509083 \\
cmpsb & 0.00000000000000 & 0.00007056742193 & 0.00000000000000 \\
\hline
\end{tabular}




\section{Appendix C: Built-in Dead code instructions}

Built-in dead code leveraged from [5]
Transfer Dead Code
1. $\operatorname{mov} R, R$
2. push $\mathrm{R}$ followed by pop $\mathrm{R}$

Arithmetic Dead Code

1. add $\mathrm{R}, 0$

2. sub $\mathrm{R}, 0$

3. adc bx, 0

4. $\mathrm{sbb} b x, 0$

5. inc $\mathrm{R}$ followed by dec $\mathrm{R}$

Logical Dead Code

1. shl R, 0

2. $\operatorname{shr} \mathrm{R}, 0$

3. and $\mathrm{R}, 1$

4. test R, 1

5. or $\mathrm{R}, 0$

6. xor $\mathrm{R}, 0$

Floating Point Dead Code

1. fadd $s t 2$, sto

2. fmul st 2, sto

3. fld st 2

4. fsub st2, sto

5. fdiv st 2, st0

6. fst st 3

Miscellaneous Dead Code

1. nop

2. neg $R$, not $R$, dec $R$ 


\section{Appendix D: Equivalent instruction substitution}

Following equivalent instruction substitutions are leveraged from [5].

Notations:

$\mathrm{R}$ - Register (eax, ax, ah, al)

$\mathrm{RR}$ - Random register

mem, [mem] - Memory address ([esi])

imm - Immediate value (12h)

op1 - To-operand with length more than 1 including $\mathrm{R}$ and mem

op 2 - From-operand with length more than 1 including R, mem, and imm

loc - any location or label

\begin{tabular}{|c|c|}
\hline add $\mathrm{R}$, imm & $\begin{array}{l}\text { 3. sub } R, \text { new_imm where new_imm }=i m m \times(-1) \\
\text { 4. lea } R \text {. }[R+\text { imm] }\end{array}$ \\
\hline $\operatorname{add} \mathrm{R}, 1$ & $\begin{array}{l}\text { not } \mathrm{R} \\
\text { neg } \mathrm{R}\end{array}$ \\
\hline mov R, imm & $\begin{array}{l}\text { 1. } \text { mov } R \text {, random_imm } \\
\text { add } R \text {, new_imm where new_imm }=i m m-\text { random_imm } \\
\text { mov } R \text {, random_imm } \\
\text { sub } R \text {, new_imm where new_imm }=(\text { random_imm - imm }) \\
\text { mov } R \text {, random_imm } \\
\text { xor } R \text {, new imm }\end{array}$ \\
\hline $\begin{array}{l}\text { mov R1, R2 } \\
\text { (no } 8 \text { bit R) }\end{array}$ & $\begin{array}{l}\text { 1. } \text { push R2 } \\
\text { pop R1 }\end{array}$ \\
\hline $\begin{array}{l}\text { mov } R, \text { mem } \\
\text { (no } 8 \text { bit } R \text { ) }\end{array}$ & $\begin{array}{l}\text { 1. push mem } \\
\text { pop R }\end{array}$ \\
\hline $\begin{array}{l}\text { mov R, imm } \\
\text { (no } 8 \text { bit R) }\end{array}$ & $\begin{array}{ll}\text { 1. } & \text { push imm } \\
\text { pop R } \\
\text { 2. lea } \mathrm{R}, \text { [imm] }\end{array}$ \\
\hline $\begin{array}{l}\text { mov mem, } R \\
\text { (no } 8 \text { bit } R \text { ) }\end{array}$ & $\begin{array}{l}\text { 1. push } \mathrm{R} \\
\text { pop mem }\end{array}$ \\
\hline mov mem, imm & $\begin{array}{l}\text { 1. } \text { push imm } \\
\text { pop mem }\end{array}$ \\
\hline cmp R, 0 & $\begin{array}{ll}\text { 1. } & \text { or } \mathrm{R}, \mathrm{R} \\
\text { 2. } & \text { and } \mathrm{R}, \mathrm{R} \\
\text { 3. } & \text { test } \mathrm{R}, \mathrm{R}\end{array}$ \\
\hline cmp R1, R2 & 1. sub R1, R2 \\
\hline cmp R, mem & 1. sub R, mem \\
\hline $\mathrm{cmp} \mathrm{R,imm}$ & 1. sub R, imm \\
\hline $\mathrm{cmp}$ mem, $\mathrm{R}$ & 1. sub mem, R \\
\hline cmp mem, imm & 1. sub mem, imm \\
\hline and $\mathrm{R} 1, \mathrm{R} 2$ & $\begin{array}{l}\text { 1. push RR } \\
\text { mov R, R1 }\end{array}$ \\
\hline
\end{tabular}




\begin{tabular}{|c|c|}
\hline & $\begin{array}{ll} & \text { or } \mathrm{R}, \mathrm{R} 2 \\
& \text { xor } \mathrm{R} 1, \mathrm{R} 2 \\
& \text { xor } \mathrm{R} 1, \mathrm{R} \\
& \text { pop } \mathrm{RR} \\
2 . & \operatorname{not} \mathrm{R} 1 \\
& \operatorname{not} \mathrm{R} 2 \\
& \text { or } \mathrm{R} 1, \mathrm{R} 2 \\
& \text { not } \mathrm{R} 1 \\
\end{array}$ \\
\hline $\operatorname{dec} \mathrm{R}$ & $\begin{array}{l}\text { 1. } \begin{array}{l}\text { neg } R \\
\text { not } R\end{array} \\
\end{array}$ \\
\hline dec mem & $\begin{array}{l}\text { 1. neg mem } \\
\text { not mem }\end{array}$ \\
\hline inc $R$ & $\begin{array}{ll}\text { 1. } & \text { add } R, 1 \\
\text { 2. } & \text { not } \mathrm{R} \\
& \operatorname{neg} \mathrm{R}\end{array}$ \\
\hline inc mem & $\begin{array}{l}\text { 1. add mem, } 1 \\
\text { 2. not mem } \\
\text { neg mem }\end{array}$ \\
\hline invoke op1, op2 & 1. stdcall [op1], op2 \\
\hline jmp loc & $\begin{array}{l}\text { 1. } \\
\text { jz loc } R R, R R\end{array}$ \\
\hline jmp R & $\begin{array}{l}\text { 1. push } \mathrm{R} \\
\text { ret }\end{array}$ \\
\hline lea $R,[R 1+R 2]$ & $\begin{array}{l}\text { 1. mov R, R1 } \\
\text { add R, R2 }\end{array}$ \\
\hline lea $\mathrm{R},[\mathrm{R}+\mathrm{R} 1+\mathrm{imm}]$ & $\begin{array}{l}\text { 1. add } \mathrm{R}, \mathrm{imm} \\
\text { add } \mathrm{R}, \mathrm{R} 1\end{array}$ \\
\hline lea $\mathrm{R},[\mathrm{R} 1+\mathrm{R} 2+\mathrm{imm}]$ & $\begin{array}{l}\text { 1. lea } \mathrm{R},[\mathrm{R} 1+\mathrm{imm}] \\
\text { add } \mathrm{R}, \mathrm{R} 2 \\
\end{array}$ \\
\hline lodsb & $\begin{array}{l}\text { 1. mov al, [esi] } \\
\text { add esi, } 1\end{array}$ \\
\hline lodsd & $\begin{array}{l}\text { 1. mov eax, [esi] } \\
\text { add esi, } 4\end{array}$ \\
\hline movsb & $\begin{array}{l}\text { 1. push eax } \\
\text { mov al, [esi] } \\
\text { add esi, } 1 \\
\text { mov [edi], al } \\
\text { add edi, } 1 \\
\text { pop eax }\end{array}$ \\
\hline movsd & $\begin{array}{l}\text { 1. } \\
\text { mosh eax [eax], esi } \\
\text { add esi, } 4 \\
\text { mov [edi], eax }\end{array}$ \\
\hline
\end{tabular}




\begin{tabular}{|c|c|}
\hline & $\begin{array}{l}\text { add edi, } 4 \\
\text { pop eax }\end{array}$ \\
\hline neg $R$ & $\begin{array}{l}\text { 1. } \operatorname{not} \mathrm{R} \\
\text { add } \mathrm{R}, 1\end{array}$ \\
\hline neg mem & $\begin{array}{l}\text { 1. not mem } \\
\text { add mem, } 1\end{array}$ \\
\hline not R & $\begin{aligned} & \text { 1. } \operatorname{neg} \mathrm{R} \\
& \text { sub } \mathrm{R}, 1 \\
& \text { 2. } \operatorname{neg} \mathrm{R} \\
& \operatorname{dec} \mathrm{R} \\
& \text { 3. } \operatorname{neg} \mathrm{R} \\
& \text { add } \mathrm{R},-1 \\
& \text { 4. } \text { xor } \mathrm{R},-1 \\
&\end{aligned}$ \\
\hline not mem & $\begin{array}{l}\text { 1. neg mem } \\
\text { sub mem, } 1 \\
\text { 2. neg mem } \\
\text { dec mem } \\
\text { 3. neg mem } \\
\text { add mem, -1 }\end{array}$ \\
\hline or $\mathrm{R} 1, \mathrm{R} 2$ & $\begin{array}{l}\text { 1. } \\
\text { movs } R R \\
\text { xor } R R, R 1 \\
\text { and } R 1, R 2 \\
\text { and } 1, R 2 \\
\text { xor } R 1, R R \\
\text { pop } R R\end{array}$ \\
\hline or $\mathrm{R} 1, \mathrm{mem}$ & $\begin{array}{l}\text { 1. } \\
\text { movsh RR } R, R 1 \\
\text { xor RR, mem } \\
\text { and R1, mem } \\
\text { xor R1, RR } \\
\text { pop RR } \\
\end{array}$ \\
\hline or $\mathrm{R} 1, \mathrm{imm}$ & $\begin{array}{l}\text { 1. } \\
\text { movh RR } R \text { R, R1 } \\
\text { xor RR, imm } \\
\text { and R1, imm } \\
\text { xor R1, RR } \\
\text { pop RR }\end{array}$ \\
\hline or mem, $\mathrm{R}$ & $\begin{array}{l}\text { 1. } \\
\text { mosh } R R \\
\text { mov } R R, \text { mem } \\
\text { xor } R R, R \\
\text { and mem, R } \\
\text { xor mem, RR } \\
\text { pop } R R\end{array}$ \\
\hline
\end{tabular}




\begin{tabular}{|c|c|}
\hline or mem, imm & $\begin{array}{l}\text { 1. } \text { push } \mathrm{RR} \\
\text { mov } \mathrm{RR}, \mathrm{mem} \\
\text { xor } \mathrm{RR}, \mathrm{imm} \\
\text { and mem, imm } \\
\text { xor mem, } \mathrm{RR} \\
\text { pop } \mathrm{RR} \\
\end{array}$ \\
\hline popad & $\begin{array}{l}\text { 1. } \text { pop edi } \\
\text { pop esi } \\
\text { pop ebp } \\
\text { add esp, } 4 \\
\text { pop ebx } \\
\text { pop edx } \\
\text { pop ecx } \\
\text { pop eax }\end{array}$ \\
\hline stdcall op1, op2 & 1. invoke [op1], op2 \\
\hline stosb & $\begin{array}{l}\text { 1. mov edi, [al] } \\
\text { add edi, } 1\end{array}$ \\
\hline stosd & $\begin{array}{l}\text { 1. mov edi, [eax] } \\
\text { add edi, } 4\end{array}$ \\
\hline sub $\mathrm{R}, \mathrm{imm}$ & 1. add $\mathrm{R}$, new imm where new imm $=\mathrm{imm} \times(-1)$ \\
\hline sub mem, imm & 1. add mem, new imm where new imm $=$ imm $x(-1)$ \\
\hline sub R, 1 & $\begin{array}{l}\text { 1. } \begin{array}{l}\text { neg R } \\
\text { not R }\end{array} \\
\end{array}$ \\
\hline sub mem, 1 & $\begin{array}{l}\text { 1. neg mem } \\
\text { not mem }\end{array}$ \\
\hline test R1, R2 & 1. or $\mathrm{R} 1, \mathrm{R} 2$ \\
\hline xchg R1, R2 & $\begin{array}{r}\text { xor } \mathrm{R} 1, \mathrm{R} 2 \\
\text { xor } \mathrm{R} 2, \mathrm{R} 1 \\
\text { xor } \mathrm{R} 1, \mathrm{R} 2\end{array}$ \\
\hline xor $\mathrm{R}, \mathrm{R}$ & $\begin{array}{ll}\text { 1. } & \text { sub } \mathrm{R}, \mathrm{R} \\
\text { 2. } & \text { mov } \mathrm{R}, 0 \\
\text { 3. } & \text { and } \mathrm{R}, 0\end{array}$ \\
\hline
\end{tabular}

